\title{
RESOLUÇÃO DE PROBLEMAS DE GEOMETRIA EUCLIDIANA UTILIZANDO TÉCNICAS DE GEOMETRIA PROJETIVA
}

FRANCISCO DIEGO FEITOSA DE SOUZA

CAMILA DE SOUSA FRANCO

DENILSO IBIAPINO FONTES

AIRTON PEREIRA DA SILVA LEÃO

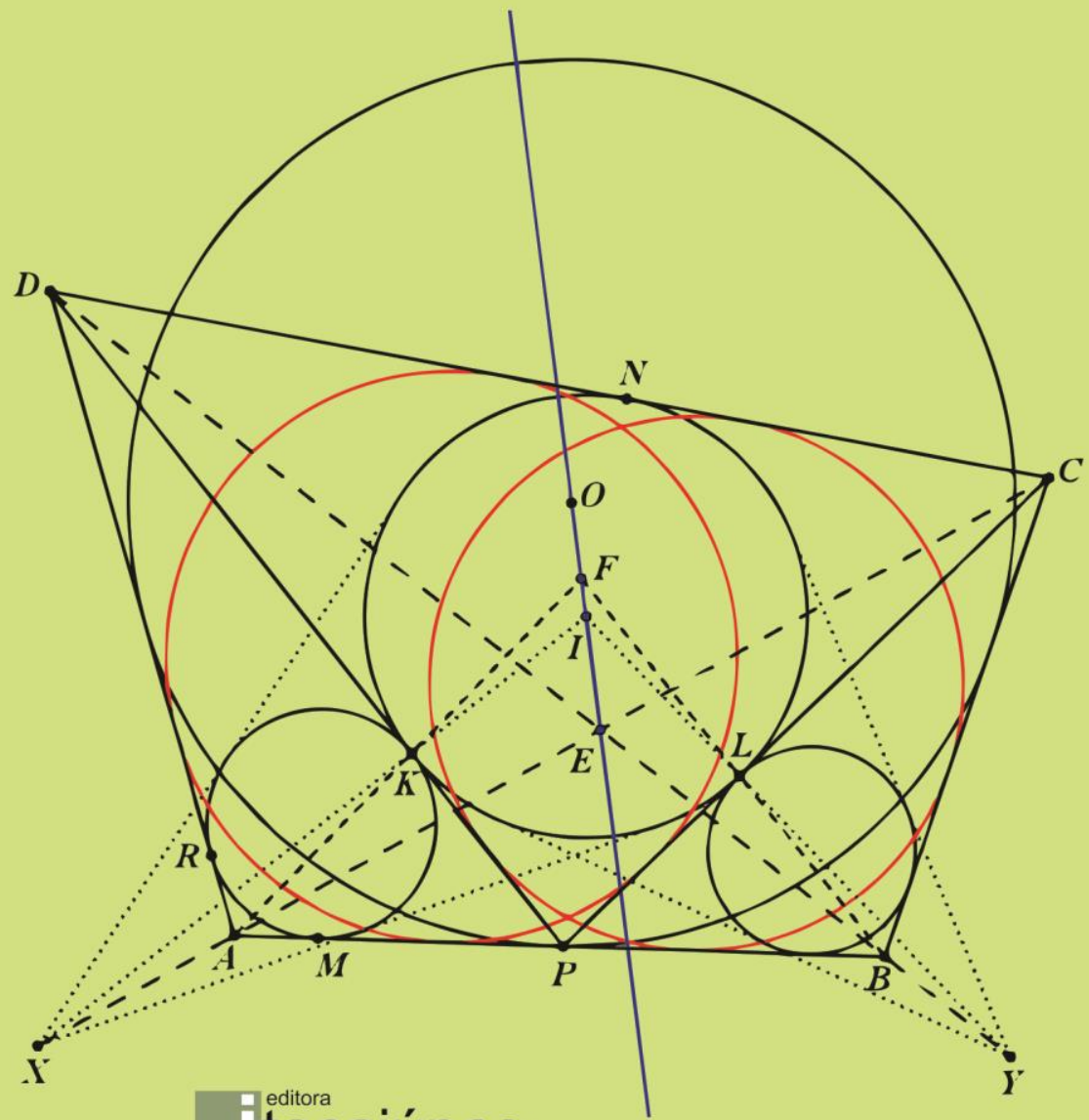

tacaiúnas 


\section{Francisco Diego Feitosa de Souza Camila de Sousa Franco Denilso Ibiapino Fontes Airton Pereira Da Silva Leão}

\section{RESOLUÇÃO DE PROBLEMAS DE GEOMETRIA EUCLIDIANA UTILIZANDO TÉCNICAS DE GEOMETRIA PROJETIVA}

$1^{\mathrm{a}}$ edição 
(C) 2020 por Francisco Diego Feitosa de Souza, Camila de Souza Franco, Denilso Ibiapino Fontes e Airton Pereira da Silva Leão

Todos os direitos reservados

\section{Conselho Editorial}

Márcia Aparecida da Silva Pimentel | Universidade Federal do Pará, Brasil José Antônio Herrera | Universidade Federal do Pará, Brasil

Bruno Nunes Batista | Instituto Federal Catarinense, Brasil

Wildoberto Batista Gurgel | Universidade Federal Rural do Semi-Árido, Brasil

André Luiz de Oliveira Brum | Universidade Federal do Rondônia, Brasil

Mariana Neves Cruz Mello | Universidade do Estado do Pará, Brasil

Josimar dos Santos Medeiros | Universidade Estadual da Paraíba, Brasil

Miguel Rodrigues Netto | Universidade do Estado de Mato Grosso, Brasil

Rodrigo de Lima Leal | Instituto de Educação Superior Raimundo Sá, Brasil

Jenaldo Alves de Araújo | Universidade Luterana do Brasil, Brasil

Mário Silva Uacane | Universidade Licungo, Moçambique

Francisco da Silva Costa | Universidade do Minho, Portugal

Ofelia Pérez Montero | Universidad de Oriente- Santiago de Cuba, Cuba

Editora-chefe: Viviane Corrêa Santos | Universidade do Estado do Pará, Brasil

Diagramação e capa: Walter Rodrigues

Dados Internacionais de Catalogação na Publicação (CIP) de acordo com ISBD

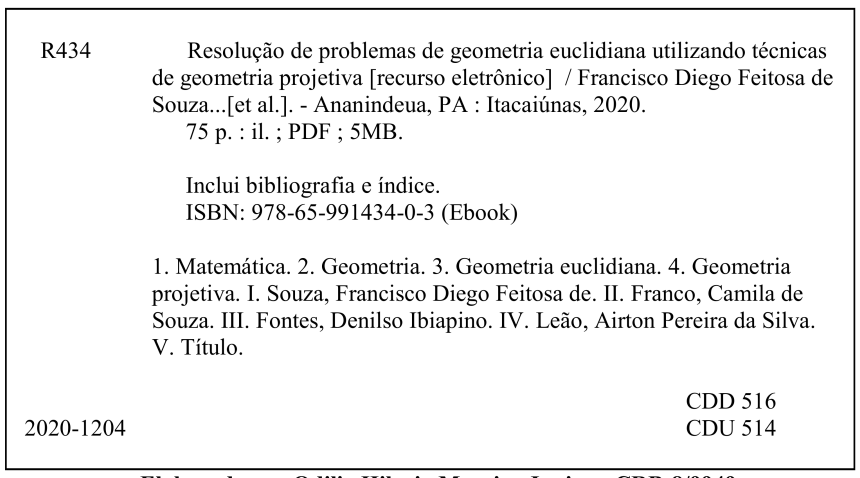

Elaborado por Odilio Hilario Moreira Junior - CRB-8/9949

Índice para catálogo sistemático:

1. Matemática : Geometria 516

2. Matemática : Geometria 514

DOI: 10.36599/itac-ed1.011

O conteúdo desta obra, inclusive sua revisão ortográfica e gramatical, bem como os dados apresentados, é de responsabilidade de seus participantes, detentores dos Direitos Autorais. 
A Deus.

Aos meus pais, Antônio Ribeiro de Souza e Maria Iramy Feitosa de Souza. 
"A Geometria existe, como já disse o filósofo, por toda a parte. É preciso, porém, olhos para vê-la, inteligência para compreendê-la e alma para admirá-la."

(Júlio César de Mello e Souza) 


\section{S U M Á R I O}

1. INTRODUÇÃO .7

2. TÉCNICAS DE GEOMETRIA PROJETIVA.............9

3. SOLUÇÃO DOS PROBLEMAS....................................41

4. CONSIDERAÇÕES FINAIS.........................................73 


\section{INTRODUÇÃO}

O presente livro se propõe a resolver problemas de Geometria Euclidiana utilizando técnicas de Geometria Projetiva.

A Geometria é parte integrante de currículos escolares e importante em aplicações práticas do cotidiano. Sendo assim, o saber geométrico se faz de forma gradativa, buscando estimular a noção de espaço e os problemas colocados pelo conhecimento deste. Historicamente, a Geometria Projetiva surge junto ao Movimento Renascentista, justamente pelo fato dos artistas buscarem mais realismo em suas obras, introduzindo conceitos de ponto de fuga e perspectividade. Esta é uma alternativa à Geometria Euclidiana.

Fazendo uma analogia entre as duas, a Euclidiana se preocupa com o mundo em que vivemos e a Projetiva com o mundo que vemos, sendo denominada, por vezes, como a geometria visual. Outro aspecto de diferenciação entre as duas, é que, contrariamente à primeira, o desenvolvimento da Geometria Projetiva pode ser feito utilizando-se apenas uma régua. Esta tem como característica marcante a seguinte: duas retas quaisquer no plano sempre se intersectam.

É importante traçar esse paralelo, pois este livro consiste na resolução de problemas da Geometria Euclidiana através da Geometria Projetiva. Esse processo se dá, inicialmente, com as definições de alguns conceitos de Geometria Projetiva e, a partir destes, obtendo proposições e teoremas. Na primeira seção definimos razão cruzada, conjuntos harmônicos, perspectividade, projetividade, pólo e polar. Enunciamos e demonstramos algumas proposições e teoremas. Destacamos os Teoremas de Desargues, Monge, Monge e D'Alembert, Pappus, Pascal, Brianchon e Brocard. Na 
segunda seção resolvemos vários problemas de Geometria Euclidiana, entre estes alguns de Olimpíadas de Matemática, utilizando as técnicas de Geometria Projetiva desenvolvida na primeira seção.

A Geometria Projetiva é uma teoria geométrica independente, com seu próprio conjunto de axiomas. Enfatizamos que o livro apresentado não se propõe a provar os teoremas e resolver problemas da Geometria Projetiva, mas sim, aplicar suas técnicas na resolução de problemas da Geometria Euclidiana.

A relevância do estudo realizado se dá na aplicabilidade didática para discentes de Olimpíadas Matemática, visando uma ferramenta alternativa para a resolução de problemas de Geometria Euclidiana.

Esperamos que o texto discorrido no livro sirva como base preparatória para competições matemáticas, visto a decorrência de problemas envolvendo Geometria. 


\section{TÉCNICAS DE GEOMETRIA PROJETIVA}

A matemática é uma ciência que surgiu essencialmente da necessidade de resolução de problemas. A geometria é uma palavra de origem grega. Geo significa terra, e metria, que vem da palavra métron, significa medir. Sendo assim, é um campo que se dedica a estudar as medidas das formas de figuras planas ou espaciais, bem como sobre a posição relativa das figuras no espaço e suas propriedades. O seu surgimento vem associado à resolução de problemas práticos, como a medição de terrenos. Passando especificamente para a Geometria Projetiva, esta surge na Itália, no século XV, aliada ao movimento Renascentista, no qual os artistas buscavam mais realismo para suas obras, introduzindo conceitos de perspectividade. Porém, somente dois séculos mais tarde se formulam as ideias matematicamente.

A figura pioneira e precursora da Geometria Projetiva é Girard Desargues, matemático e arquiteto francês. Em 1639, apresenta trabalho sobre a teoria de Geometria das Cônicas, Broullion Projet, formalizando esses conceitos. Porém, suas ideias não são bem aceitas, principalmente porque o mesmo se utiliza de uma linguagem peculiar. Entretanto, elas são resgatadas dois séculos mais tarde por Jean Victor Poncelet.

Nesta seção será descrita uma série de definições, proposições e teoremas que servirão de base para a resolução de problemas da próxima seção. Destacamos os teoremas de Desargues, Monge, Monge e D’Alembert, Pappus, Pascal, Brianchon e Brocard, os quais são ferramentas importantes para o estudo proposto nesse livro. 
Definição 1. Sejam $A, B, C$ e $D$ quatro pontos distintos incidentes numa reta orientada $r$. Nesta ordem, a razão cruzada de $A, B$ em relação a $C, D$ representada por $R(A, B ; C, D)$, é definida por

$$
R(A, B ; C, D)=\frac{\overline{A C}}{\overline{C B}} \frac{\overline{D B}}{\overline{A D}},
$$

onde os comprimentos são direcionados.

Proposição 1. Sejam $a, b, c$ e $d$ quatro retas concorrentes no ponto $O$. Para as retas $p_{1}$ e $p_{2}$ que não contêm $O$, denotamos $A_{i}=a \cap p_{i}, \quad B_{i}=b \cap p_{i}, \quad C_{i}=c \cap p_{i}$ e $D_{i}=d \cap p_{i}, \quad$ para $i=1$, 2. Então

$$
R\left(A_{1}, B_{1} ; C_{1}, D_{1}\right)=R\left(A_{2}, B_{2} ; C_{2}, D_{2}\right) .
$$

\section{Demonstração.}

Sem perda de generalidade, suponha que os pontos $A_{1}, C_{1}, B_{1}$ e $D_{1}$ são colineares nessa ordem. Aplicando a lei dos senos nos triângulos $C_{1} O A_{1}, \quad C_{1} O B_{1}, D_{1} O B_{1}$ e $D_{1} O A_{1}$, obtemos respectivamente

$$
\begin{gathered}
\overline{A_{1} C_{1}}=\frac{\overline{O C_{1}} \cdot \operatorname{sen}\left(\angle A_{1} O C_{1}\right)}{\operatorname{sen}\left(\angle C_{1} A_{1} O\right)}, \overline{C_{1} B_{1}}=\frac{\overline{O C_{1}} \cdot \operatorname{sen}\left(\angle C_{1} O B_{1}\right)}{\operatorname{sen}\left(\angle O B_{1} C_{1}\right)}, \\
\overline{D_{1} B_{1}}=\frac{\overline{D_{1} O} \cdot \operatorname{sen}\left(\angle D_{1} O B_{1}\right)}{\operatorname{sen}\left(\angle D_{1} B_{1} O\right)} \text { e } \overline{A_{1} D_{1}}=\frac{\overline{D_{1} O} \cdot \operatorname{sen}\left(\angle A_{1} O D_{1}\right)}{\operatorname{sen}\left(\angle D_{1} A_{1} O\right)},
\end{gathered}
$$

onde os comprimentos e os ângulos são direcionados.

Pela Definição 1, temos

$$
\begin{aligned}
& R\left(A_{1}, B_{1} ; C_{1}, D_{1}\right)=\overline{\overline{A_{1} C_{1}}} \overline{\overline{C_{1} B_{1}}} \frac{\overline{D_{1} B_{1}}}{\overline{A_{1} D_{1}}} \\
& =\frac{\overline{O C_{1}} \cdot \operatorname{sen}\left(\angle A_{1} O C_{1}\right)}{\frac{\operatorname{sen}\left(\angle C_{1} A_{1} O\right)}{\overline{O C_{1}} \cdot \operatorname{sen}\left(\angle C_{1} O B_{1}\right)}} \frac{\overline{D_{1} O} \cdot \operatorname{sen}\left(\angle D_{1} O B_{1}\right)}{\operatorname{sen}\left(\angle D_{1} B_{1} O\right)} \\
& \frac{\overline{D_{1} O} \cdot \operatorname{sen}\left(\angle A_{1} O D_{1}\right)}{\operatorname{sen}\left(\angle O B_{1} C_{1}\right)}
\end{aligned}
$$




$$
\begin{aligned}
& =\frac{\operatorname{sen}\left(\angle A_{1} O C_{1}\right)}{\operatorname{sen}\left(\angle C_{1} O B_{1}\right)} \frac{\operatorname{sen}\left(\angle D_{1} O B_{1}\right)}{\operatorname{sen}\left(\angle A_{1} O D_{1}\right)} \frac{\operatorname{sen}\left(\angle O B_{1} C_{1}\right)}{\operatorname{sen}\left(\angle D_{1} B_{1} O\right)} \frac{\operatorname{sen}\left(\angle D_{1} A_{1} O\right)}{\operatorname{sen}\left(\angle C_{1} A_{1} O\right)} \\
& =\frac{\operatorname{sen}\left(\angle A_{1} O C_{1}\right)}{\operatorname{sen}\left(\angle C_{1} O B_{1}\right)} \frac{\operatorname{sen}\left(\angle D_{1} O B_{1}\right)}{\operatorname{sen}\left(\angle A_{1} O D_{1}\right)} \frac{\operatorname{sen}\left(\pi-\angle D_{1} B_{1} O\right)}{\operatorname{sen}\left(\angle D_{1} B_{1} O\right)} \frac{\operatorname{sen}\left(\angle C_{1} A_{1} O\right)}{\operatorname{sen}\left(\angle C_{1} A_{1} O\right)} \\
& =\frac{\operatorname{sen}\left(\angle A_{1} O C_{1}\right)}{\operatorname{sen}\left(\angle C_{1} O B_{1}\right)} \frac{\operatorname{sen}\left(\angle D_{1} O B_{1}\right)}{\operatorname{sen}\left(\angle A_{1} O D_{1}\right)}
\end{aligned}
$$

Analogamente, aplicando a lei dos senos nos triângulos $\mathrm{C}_{2} \mathrm{OA}_{2}$, $\mathrm{C}_{2} \mathrm{OB}_{2}, \mathrm{D}_{2} \mathrm{OA}_{2}$ e $\mathrm{D}_{2} O \mathrm{~B}_{2}$, obtemos

$$
\begin{aligned}
R\left(A_{2}, B_{2} ; C_{2}, D_{2}\right) & =\frac{\operatorname{sen}\left(\angle A_{2} O C_{2}\right)}{\operatorname{sen}\left(\angle C_{2} O B_{2}\right)} \frac{\operatorname{sen}\left(\angle D_{2} O B_{2}\right)}{\operatorname{sen}\left(\angle A_{2} O D_{2}\right)} \\
& =\frac{\operatorname{sen}\left(\angle A_{1} O C_{1}\right)}{\operatorname{sen}\left(\angle C_{1} O B_{1}\right)} \frac{\operatorname{sen}\left(\angle D_{1} O B_{1}\right)}{\operatorname{sen}\left(\angle A_{1} O D_{1}\right)} .
\end{aligned}
$$

Portanto,

$$
R\left(A_{1}, B_{1} ; C_{1}, D_{1}\right)=R\left(A_{2}, B_{2} ; C_{2}, D_{2}\right) .
$$

\section{Proposição 1}

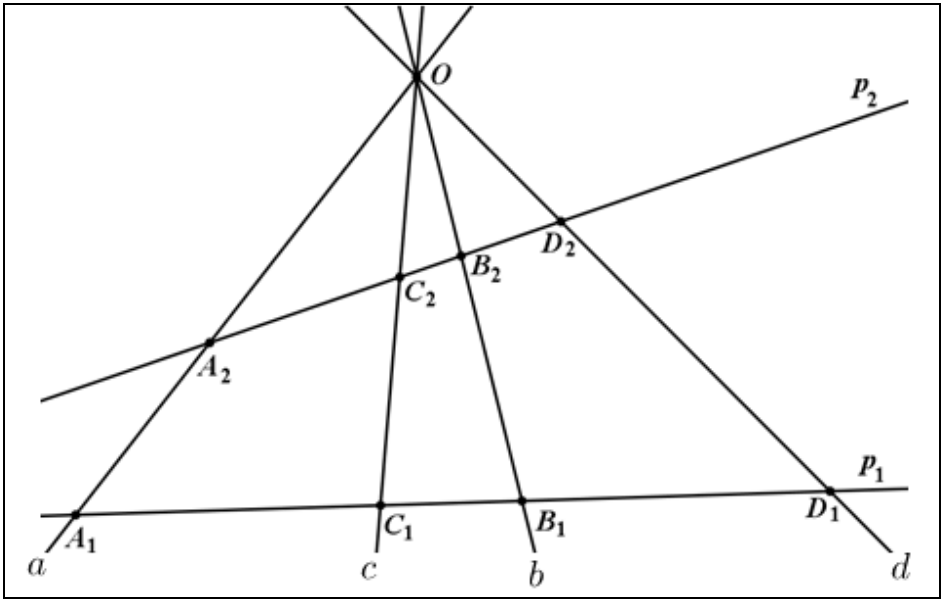

A proposição acima mostra que a razão cruzada dos pontos $A_{1}, B_{1}, C_{1}$ e $D_{1}$ é invariante em relação ao feixe de retas $a, b, c$ 
e $d$. Com isso, vamos definir a razão cruzada de um feixe de quatro retas da seguinte forma:

Definição 2. Sejam $a, b, c$ e $d$ quatro retas concorrente no ponto $O$. Seja $p$ uma reta que não contém $O$ e intersecta as retas $a, b, c$ e $d$ nos pontos $A, B, C$ e $D$, respectivamente. A razão cruzada de $a, b$ em relação a $c, d$, representada por $R(a, b ; c, d)$, é definida por

$$
R(a, b ; c, d)=R(A, B ; C, D) .
$$

Proposição 2. Sejam $O_{1}, O_{2}, A, B, C$ e $D$ pontos pertencentes a uma circunferência. Então

$$
R\left(\overleftrightarrow{O_{1} A}, \overleftrightarrow{O_{1} B} ; \overleftrightarrow{O_{1} C}, \overleftrightarrow{O_{1} D}\right)=R\left(\overleftrightarrow{O_{2} A}, \overleftrightarrow{O_{2} B} ; \overleftrightarrow{O_{2} C}, \overleftrightarrow{O_{2} D}\right) \text {. }
$$

\section{Demonstração.}

Sejam $C_{1}=A D \cap O_{1} C, \quad B_{1}=A D \cap O_{1} B, \quad C_{2}=A D \cap O_{2} C$ e $B_{2}=A D \cap O_{2} B$. Primeiramente vamos demonstrar que $R\left(A, B_{1} ; C_{1}, D\right)=R\left(A, B_{2} ; C_{2}, D\right)$. Para tanto, aplicando a lei dos senos nos triângulos $A C_{1} O_{1}, C_{1} B_{1} O_{1}, B_{1} O_{1} D$ e $A O_{1} D$, obtemos respectivamente

$$
\begin{gathered}
\overline{A C_{1}}=\frac{\overline{O_{1} C_{1}} \cdot \operatorname{sen}\left(\angle A O_{1} C_{1}\right)}{\operatorname{sen}\left(\angle C_{1} A O_{1}\right)}, \overline{C_{1} B_{1}}=\frac{\overline{O_{1} C_{1}} \cdot \operatorname{sen}\left(\angle C_{1} O_{1} B_{1}\right)}{\operatorname{sen}\left(\angle O_{1} B_{1} C_{1}\right)}, \\
\overline{D B_{1}}=\frac{\overline{D O_{1}} \cdot \operatorname{sen}\left(\angle B_{1} O_{1} D\right)}{\operatorname{sen}\left(\angle D B_{1} O_{1}\right)} \text { e } \overline{A D}=\frac{\overline{D O_{1}} \cdot \operatorname{sen}\left(\angle A O_{1} D\right)}{\operatorname{sen}\left(\angle D A O_{1}\right)},
\end{gathered}
$$

onde os comprimentos e os ângulos são direcionados.

Pela Definição 1, temos

$R\left(A, B_{1} ; C_{1}, D\right)=\frac{\overline{A C_{1}}}{\overline{C_{1} B_{1}}} \frac{\overline{D B_{1}}}{\overline{A D}}$ 


$$
\begin{aligned}
& \frac{\overline{O_{1} C_{1}} \cdot \operatorname{sen}\left(\angle A O_{1} C_{1}\right)}{\operatorname{sen}\left(\angle C_{1} A O_{1}\right)} \frac{\overline{D O_{1}} \cdot \operatorname{sen}\left(\angle B_{1} O_{1} D\right)}{\frac{\operatorname{sen}\left(\angle D B_{1} O_{1}\right)}{\overline{O_{1} C_{1}} \cdot \operatorname{sen}\left(\angle C_{1} O_{1} B_{1}\right)}} \frac{\overline{D O_{1}} \cdot \operatorname{sen}\left(\angle A O_{1} D\right)}{\operatorname{sen}\left(\angle D A O_{1} B_{1} C_{1}\right)} \\
= & \frac{\operatorname{sen}\left(\angle A O_{1} C_{1}\right)}{\operatorname{sen}\left(\angle C_{1} O_{1} B_{1}\right)} \frac{\operatorname{sen}\left(\angle B_{1} O_{1} D\right)}{\operatorname{sen}\left(\angle A O_{1} D\right)} \frac{\operatorname{sen}\left(\angle O_{1} B_{1} C_{1}\right)}{\operatorname{sen}\left(\angle D B_{1} O_{1}\right)} \frac{\operatorname{sen}\left(\angle D A O_{1}\right)}{\operatorname{sen}\left(\angle C_{1} A O_{1}\right)} \\
= & \frac{\operatorname{sen}\left(\angle A O_{1} C_{1}\right)}{\operatorname{sen}\left(\angle C_{1} O_{1} B_{1}\right)} \frac{\operatorname{sen}\left(\angle B_{1} O_{1} D\right)}{\operatorname{sen}\left(\angle A O_{1} D\right)} \frac{\operatorname{sen}\left(\pi-\angle D B_{1} O_{1}\right)}{\operatorname{sen}\left(\angle D B_{1} O_{1}\right)} \frac{\operatorname{sen}\left(\angle C_{1} A O_{1}\right)}{\operatorname{sen}\left(\angle C_{1} A O_{1}\right)} \\
= & \frac{\operatorname{sen}\left(\angle A O_{1} C_{1}\right)}{\operatorname{sen}\left(\angle C_{1} O_{1} B_{1}\right)} \frac{\operatorname{sen}\left(\angle B_{1} O_{1} D\right)}{\operatorname{sen}\left(\angle A O_{1} D\right)} .
\end{aligned}
$$

Analogamente, aplicando a lei dos senos nos triângulos $\mathrm{AC}_{2} \mathrm{O}_{2}, \mathrm{C}_{2} \mathrm{~B}_{2} \mathrm{O}_{2}, \mathrm{AO}_{2} \mathrm{D}$ e $\mathrm{B}_{2} \mathrm{O}_{2} \mathrm{D}$, obtemos

$$
\begin{aligned}
R\left(A, B_{2} ; C_{2}, D\right) & =\frac{\operatorname{sen}\left(\angle A O_{2} C_{2}\right)}{\operatorname{sen}\left(\angle C_{2} O_{2} B_{2}\right)} \frac{\operatorname{sen}\left(\angle B_{2} O_{2} D\right)}{\operatorname{sen}\left(\angle A O_{2} D\right)} \\
& =\frac{\operatorname{sen}\left(\angle A O_{1} C_{1}\right)}{\operatorname{sen}\left(\angle C_{1} O_{1} B_{1}\right)} \frac{\operatorname{sen}\left(\angle B_{1} O_{1} D\right)}{\operatorname{sen}\left(\angle A O_{1} D\right)} .
\end{aligned}
$$

Com isso,

$$
R\left(A, B_{1} ; C_{1}, D\right)=R\left(A, B_{2} ; C_{2}, D\right) .
$$

Portanto, pela Definição 2,

$$
R\left(\overleftrightarrow{O_{1} A}, \overleftrightarrow{O_{1} B} ; \overleftrightarrow{O_{1} C}, \overleftrightarrow{O_{1} D}\right)=R\left(\overleftrightarrow{O_{2} A}, \overleftrightarrow{O_{2} B} ; \overleftrightarrow{O_{2} C}, \overleftrightarrow{O_{2} D}\right)
$$




\section{Proposição 2}

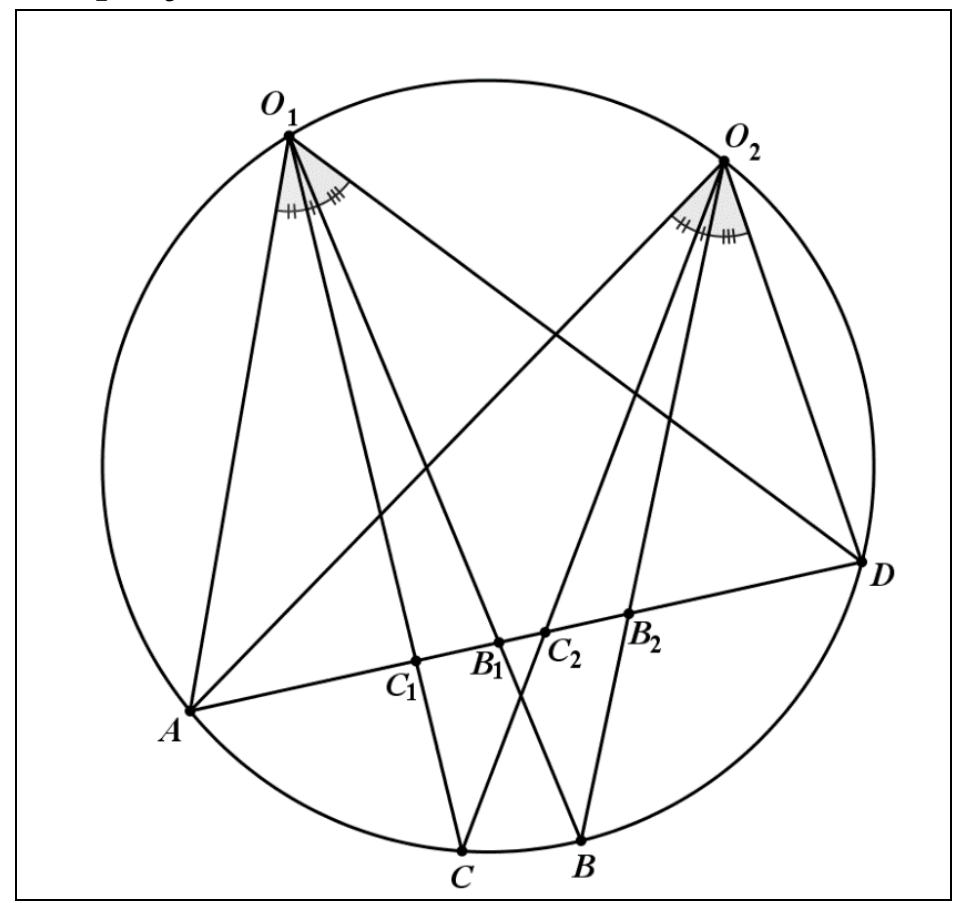

A proposição acima afirma que a razão cruzada das retas $\overleftrightarrow{O_{1} A}, \overleftrightarrow{O_{1} B}, \overleftrightarrow{O_{1} C}$ e $\overleftrightarrow{O_{1} D}$ é invariante em relação aos pontos $A$ $B, C$ e $D$. Com isso, vamos definir a razão cruzada dos quatro pontos da seguinte forma:

Definição 3. Sejam $O, A, B, C$ e $D$ pontos pertencentes a uma circunferência. Então

$$
R(A, B ; C, D)=R(\overleftrightarrow{O A}, \overleftrightarrow{O B} ; \overleftrightarrow{O C}, \overleftrightarrow{O D})
$$

Propriedades da razão cruzada. Sejam $A, B, C$ e $D$ pontos colineares ou concíclicos.

Propriedade 1. Se os pontos os pontos $A, B, C$ e $D$ são dois a dois distintos, então $R(A, B ; C, D) \neq 0$. 
Propriedade 2. Tem-se $R(A, B ; C, D)<0$ se, e somente se, os pontos $A, B, C$ e $D$ são dois a dois distintos e um dos pontos $C$, $D$ pertence a $A B(\widehat{A B})$, onde $A, B, C$ e $D$ são pontos colineares (concíclicos).

\section{Propriedade 3.}

$R(A, B ; C, D)=R(B, A ; D, C)=R(C, D ; A, B)=R(D, C ; B, A)$.

Propriedade 4. $R(A, B ; C, D)=1-R(A, C ; B, D)$.

Propriedade 5. $R(A, B ; C, D)=\frac{1}{R(A, B ; D, C)}$.

Proposição 3. Sejam $A, B, C$ e $D$ pontos concíclicos ou pontos colineares. Considere uma inversão em relação a uma circunferência de centro $O$, que mapeia os pontos $A, B, C$ e $D$ em $A^{*}, B^{*}, C^{*}$ e $D^{*}$, respectivamente. Então

$$
R(A, B ; C, D)=R\left(A^{*}, B^{*} ; C^{*}, D^{*}\right) .
$$

\section{Demonstração.}

Vamos primeiramente considerar o caso em que os pontos $A, B$, $C$ e $D$ são colineares.

Há dois subcasos:

Primeiro subcaso: $\mathrm{O}$ ponto $O$ não pertence à reta $\overleftrightarrow{A B}$.

Com isso, a inversão da reta $\overleftrightarrow{A B}$ em relação a uma circunferência de centro $O$ é uma circunferência que contém o ponto $O$. Então, os pontos $A^{*}, B^{*}, C^{*}, D^{*}$ e $O$ pertencem a esta circunferência, como mostra a figura abaixo. 


\section{Proposição 3 - $A, B, C$ e $D$ são colineares}

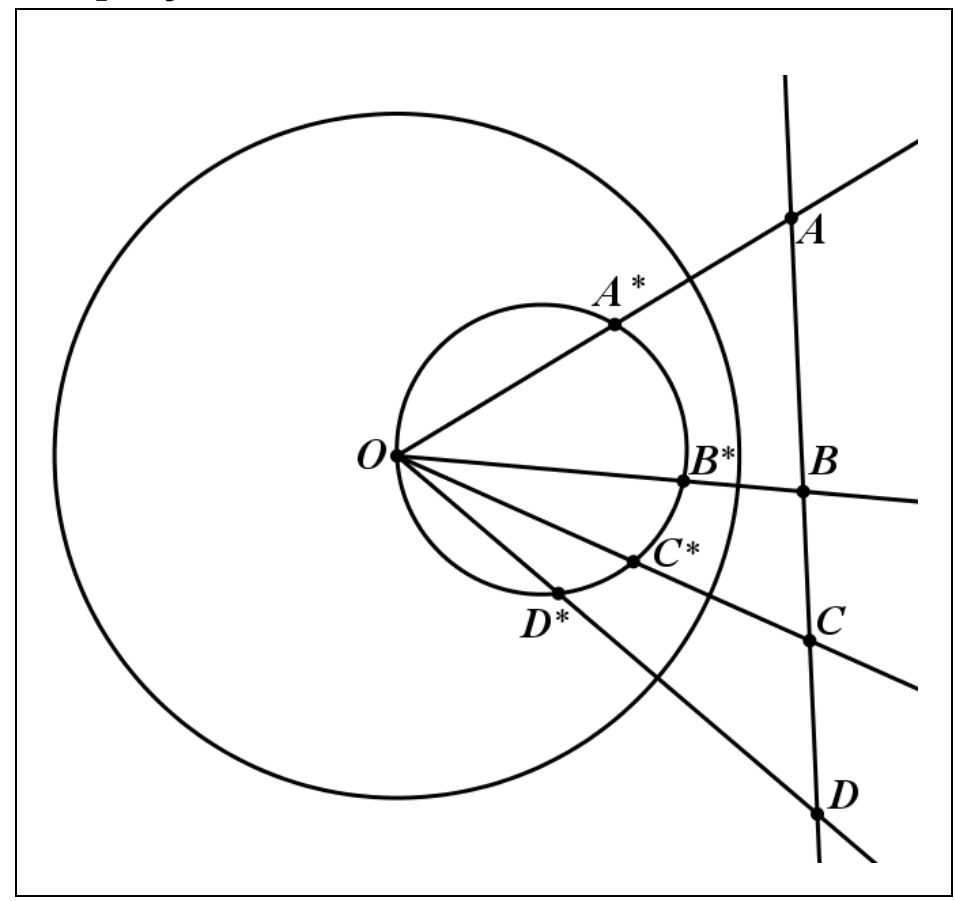

De acordo com a Definição 2, temos

$$
R\left(\overleftrightarrow{O A^{*}}, \overleftrightarrow{O B^{*}} ; \overleftrightarrow{O C^{*}}, \overleftrightarrow{O D^{*}}\right)=R(A, B ; C, D)
$$

Pela Definição 3, temos

$$
R\left(\overleftrightarrow{O A^{*}}, \overleftrightarrow{O B^{*}} ; \overleftrightarrow{O C^{*}}, \overleftrightarrow{O D^{*}}\right)=R\left(A^{*}, B^{*} ; C^{*}, D^{*}\right)
$$

Portanto,

$$
R(A, B ; C, D)=R\left(A^{*}, B^{*} ; C^{*}, D^{*}\right) .
$$

Segundo subcaso: $\mathrm{O}$ ponto $O$ pertence à reta $\overleftrightarrow{A B}$.

Com isso, a inversão da reta $\overleftrightarrow{A B}$ em relação a uma circunferência de centro $O$ é a reta $\overleftrightarrow{A B}$. Então, os pontos $A, B$, 
$C, D, A^{*}, B^{*}, C^{*}, D^{*}$ e $O$ são colineares, como ilustra a figura abaixo.

\section{Proposição 3-A, $B, C, D$ e $O$ são colineares}

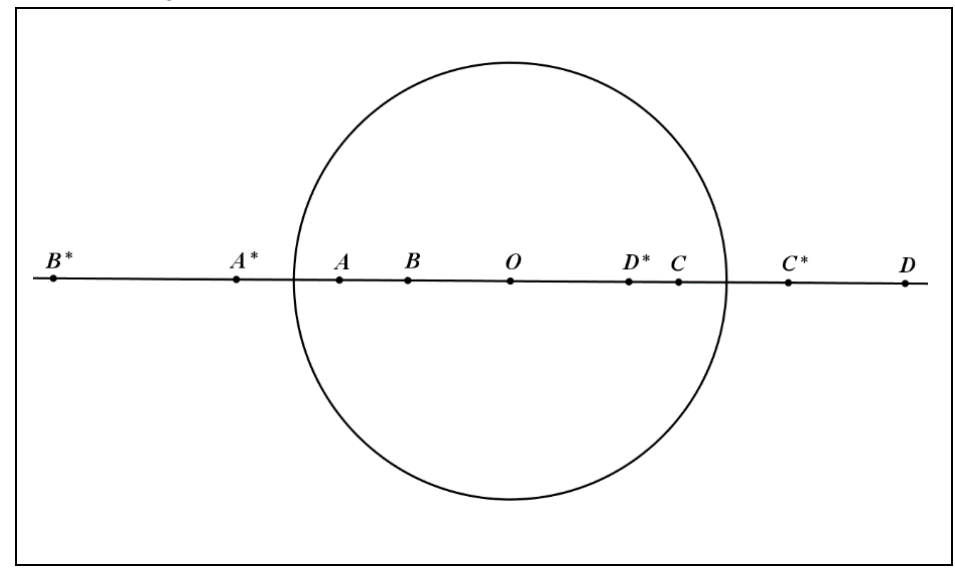

Sendo os ponto $A^{*}$ e $C^{*}$ as imagens dos pontos $A$ e $C$, respectivamente, em relação à inversão de centro $O \mathrm{e}$ considerando os comprimentos direcionados, obtemos

$$
\overline{A C}=\overline{A O}+\overline{O C}=\frac{R^{2}}{\overline{A^{*} O}}+\frac{R^{2}}{\overline{O C^{*}}}=\frac{R^{2}\left(\overline{O C^{*}}-\overline{O A^{*}}\right)}{\overline{A^{*} O} \cdot \overline{O C^{*}}}=\frac{R^{2} \cdot \overline{A^{*} C^{*}}}{\overline{A^{*} O} \cdot \overline{O C^{*}}}
$$

onde $R$ é o raio da circunferência de inversão.

Analogamente, obtemos

$$
\overline{C B}=\frac{R^{2} \cdot \overline{C^{*} B^{*}}}{\overline{C^{*} O \cdot \overline{O B^{*}}}}, \quad \overline{D B}=\frac{R^{2} \cdot \overline{D^{*} B^{*}}}{\overline{D^{*} O} \cdot \overline{O B^{*}}} \quad \text { e } \overline{A D}=\frac{R^{2} \cdot \overline{A^{*} D^{*}}}{\overline{A^{*} O} \cdot \overline{O D^{*}}} .
$$

Pela definição 1 , obtemos

$$
\begin{aligned}
R(A, B ; C, D)= & \frac{\overline{A C} \overline{\overline{D B}}}{\overline{\overline{C B}} \frac{\overline{A D}}{A D}} \\
= & \frac{\overline{R^{2} \cdot \overline{A^{*} C^{*}} \cdot \overline{O C^{*}}}}{\frac{R^{2} \cdot \overline{D^{*} B^{*}}}{\overline{D^{*} O} \cdot \overline{O B^{*} B^{*}}}} \frac{\frac{R^{2} \cdot \overline{A^{*} D^{*}}}{\overline{C^{*} O} \cdot \overline{O B^{*}}}}{\bar{A}^{*} O \cdot \overline{O D^{*}}}
\end{aligned}
$$




$$
\begin{aligned}
& =\frac{\overline{A^{*} C^{*}} \overline{D^{*} B^{*}} \overline{\overline{C^{*} O}} \overline{\overline{A^{*} O}} \overline{\overline{O D^{*}}} \overline{\overline{O B^{*}}}}{\overline{C^{*} B^{*}}} \overline{\overline{A^{*} D^{*}}} \overline{\overline{O C^{*}}} \overline{\overline{A^{*} O}} \overline{\overline{D^{*} O}} \overline{\overline{O B^{*}}} \\
& =\overline{\overline{A^{*} C^{*}}} \overline{\overline{D^{*} B^{*}}} \overline{\overline{A^{*} D^{*}}} \\
& =R\left(A^{*}, B^{*} ; C^{*}, D^{*}\right) .
\end{aligned}
$$

Agora, vamos mostrar o caso em que os pontos $A, B, C$ e $D$ pertencem a uma circunferência $C_{1}$.

Temos dois subcasos a analisar.

Primeiro subcaso: $\mathrm{O}$ ponto $O$ não pertence a circunferência $C_{1}$. Com isso, a inversão da circunferência $C_{1}$ em relação à circunferência de centro $O$ é uma circunferência que não contém o ponto $O$, como ilustra a figura abaixo.

\section{Proposição 3-A, $B, C$ e $D$ são concíclicos}

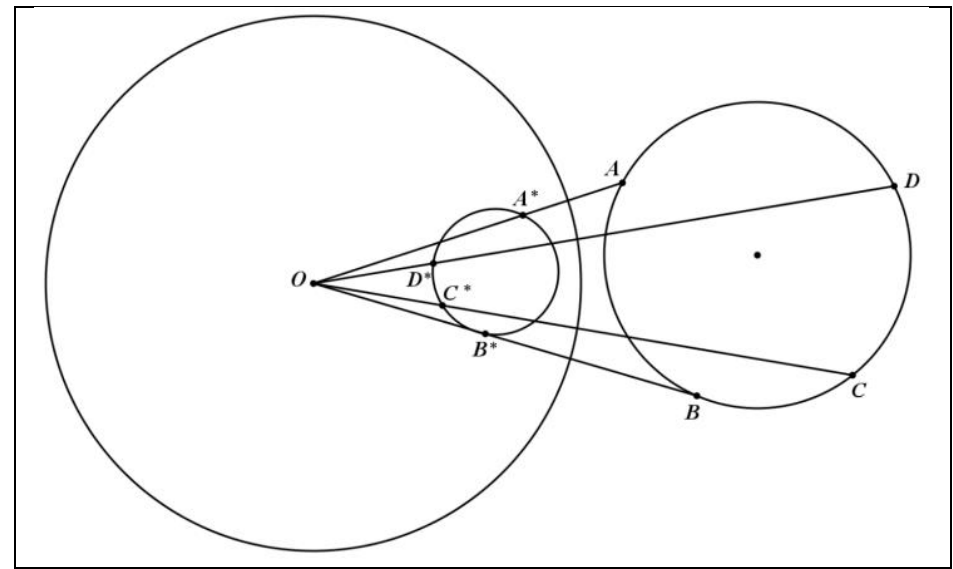

De acordo com a Definição 3, temos

$R(A, B ; C, D)=R(\overleftrightarrow{O A}, \overleftrightarrow{O B} ; \overleftrightarrow{O C}, \overleftrightarrow{O D})$ e $R\left(A^{*}, B^{*} ; C^{*}, D^{*}\right)=$ $=R\left(\overleftrightarrow{O A^{*}}, \overleftrightarrow{O B^{*}} ; \overleftrightarrow{O C^{*}}, \overleftrightarrow{O D^{*}}\right)$.

Portanto,

$$
R(A, B ; C, D)=R\left(A^{*}, B^{*} ; C^{*}, D^{*}\right) .
$$


Segundo subcaso: $\mathrm{O}$ ponto $O$ pertence à circunferência $C_{1}$.

Com isso, a inversão da circunferência $C_{1}$ em relação a uma circunferência de centro $O$ é um reta que não contém $O$, como ilustra a figura abaixo.

Proposição $3-A, B, C, D$ e $O$ são concíclicos

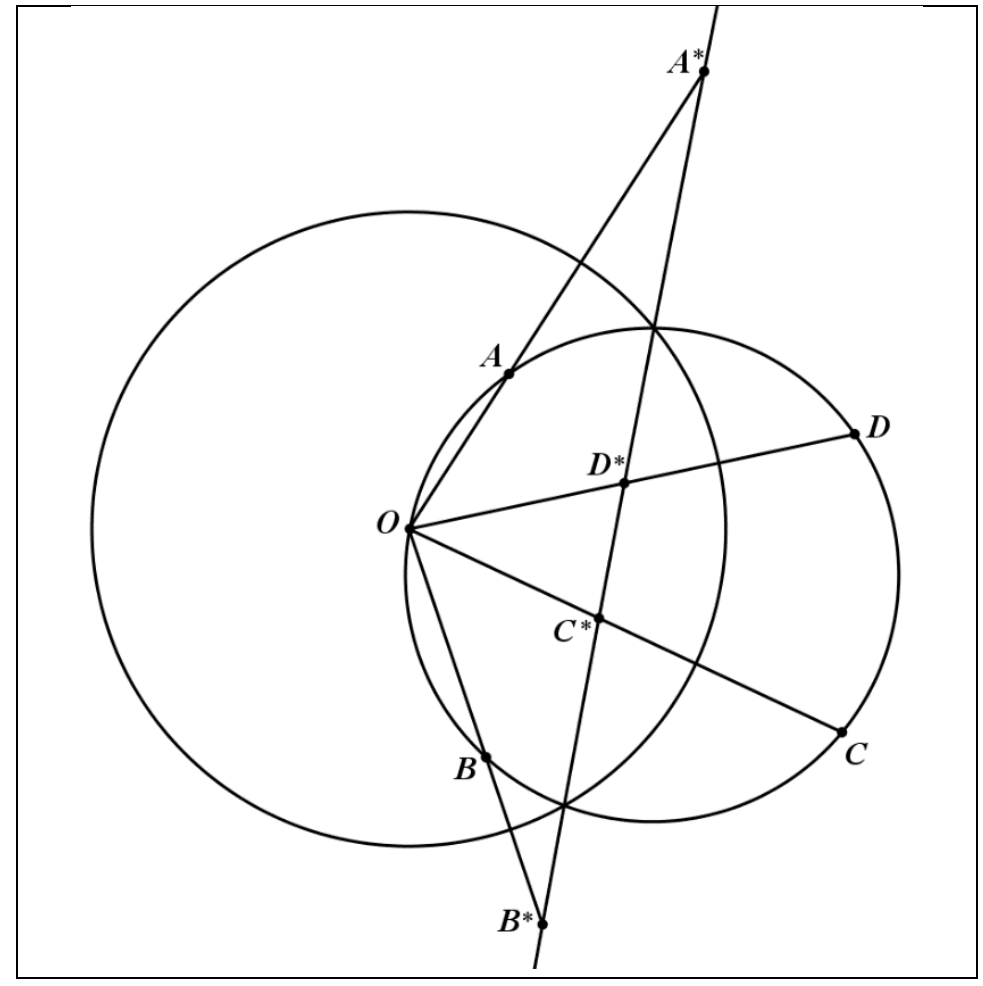

De acordo com a Definição 2, temos

$$
R(\overleftrightarrow{O A}, \overleftrightarrow{O B} ; \overleftrightarrow{O C}, \overleftrightarrow{O D})=R\left(A^{*}, B^{*} ; C^{*}, D^{*}\right)
$$

Pela Definição 3, temos

$$
R(\overleftrightarrow{O A}, \overleftrightarrow{O B} ; \overleftrightarrow{O C}, \overleftrightarrow{O D})=R(A, B ; C, D)
$$


Portanto,

$$
R(A, B ; C, D)=R\left(A^{*}, B^{*} ; C^{*}, D^{*}\right) .
$$

Definição 4. Sejam $A, B, C$ e $D$ pontos concíclicos ou colineares. Dizemos que os pares de pontos $(A, B)$ e $(C, D)$ são conjugados harmônicos se $R(A, B ; C, D)=-1$. Usamos a notação $H(A, B ; C, D)$ para indicar que os pares de pontos $(A, B)$ e $(C, D)$ são conjugados harmônicos.

Definição 5. Sejam $a, b, c$ e $d$ retas concorrentes. Dizemos que os pares de retas $(a, b)$ e $(c, d)$ são conjugados harmônicos se $R(a, b ; c, d)=-1$. Usamos a notação $H(a, b ; c, d)$ para indicar que os pares de retas $(a, b)$ e $(c, d)$ são conjugados harmônicos.

Proposição 4. Sejam $A, B, C$ e $D$ pontos colineares (pertencentes a uma circunferência $\Gamma$ ) e seja o ponto $O \notin \overleftrightarrow{A B}$ $(O \in \Gamma)$. Tem-se $H(A, B ; C, D)$ se, e somente se, $H(\overleftrightarrow{O A}, \overleftrightarrow{O B} ; \overleftrightarrow{O C}, \overleftrightarrow{O D})$.

Demonstração. Se os pontos $A, B, C$ e $D$ são colineares e o ponto $O \notin \overleftrightarrow{A B}$, então, pela Definição 2 ,

$$
R(A, B ; C, D)=R\left(\overleftrightarrow{O_{1} A}, \overleftrightarrow{O_{1} B} ; \overleftrightarrow{O_{1} C}, \overleftrightarrow{O_{1} D}\right)
$$

Se os pontos $A, B, C, D$ e $O$ concíclicos, então, pela Definição 3,

$$
R(A, B ; C, D)=R\left(\overleftrightarrow{O_{1} A}, \overleftrightarrow{O_{1} B} ; \overleftrightarrow{O_{1} C}, \overleftrightarrow{O_{1} D}\right)
$$

Portanto, $H(A, B ; C, D)$ se, e somente se,

$$
H(\overleftrightarrow{O A}, \overleftrightarrow{O B} ; \overleftrightarrow{O C}, \overleftrightarrow{O D})
$$


Definição 6. Suponha que $l_{1}$ e $l_{2}$ são tais que cada uma delas é uma reta ou uma circunferência. A perspectividade com relação

\section{$\underline{\underline{O}}$}

ao ponto $O$ (denotada por $\wedge$ ), é o mapeamento de $l_{1} \rightarrow l_{2}$, tal que

i) Se $l_{1}$ e $l_{2}$ são circunferências, então estas contêm o ponto $O$. Se apenas uma de $l_{1}, l_{2}$ é uma circunferência, então esta contém o ponto $O$.

ii) Cada ponto $A_{1} \in l_{1}$ é mapeado no ponto $A_{2}=\overleftrightarrow{O A_{1}} \cap l_{2}$.

\section{$\underline{\underline{O}}$}

A figura abaixo ilustra a perspectividade $A_{1} B_{1} C_{1} D_{1} \wedge A_{2} B_{2}$ $C_{2} D_{2}$, quando $l_{1}$ e $l_{2}$ são retas.

\section{Perspectividade entre pontos, quando $l_{1}$ e $l_{2}$ são retas}

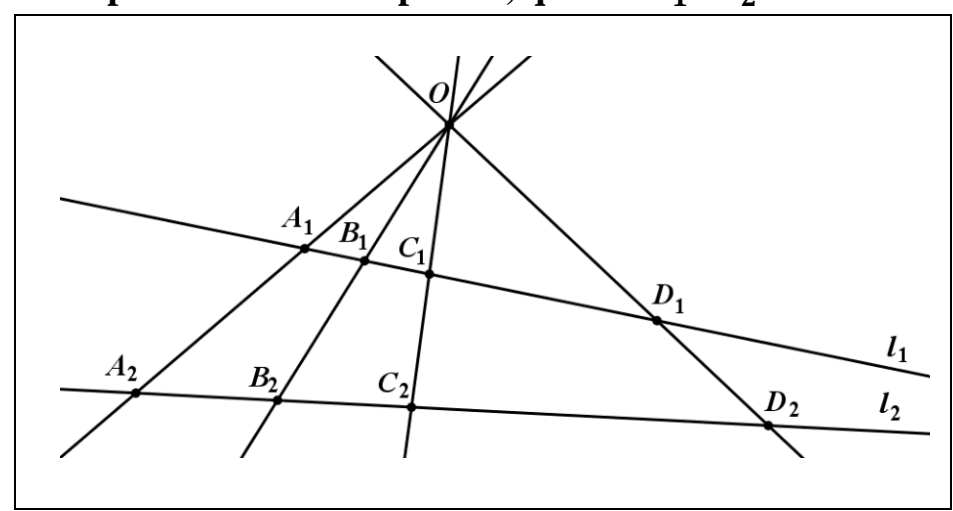

$\underline{\underline{O}}$

A figura abaixo ilustra a perspectividade $A_{1} B_{1} C_{1} D_{1} \wedge A_{2} B_{2}$ $C_{2} D_{2}$, quando $l_{1}$ e $l_{2}$ são circunferências. 
Perspectividade entre pontos, quando $l_{1}$ e $l_{2} \quad$ são circunferências

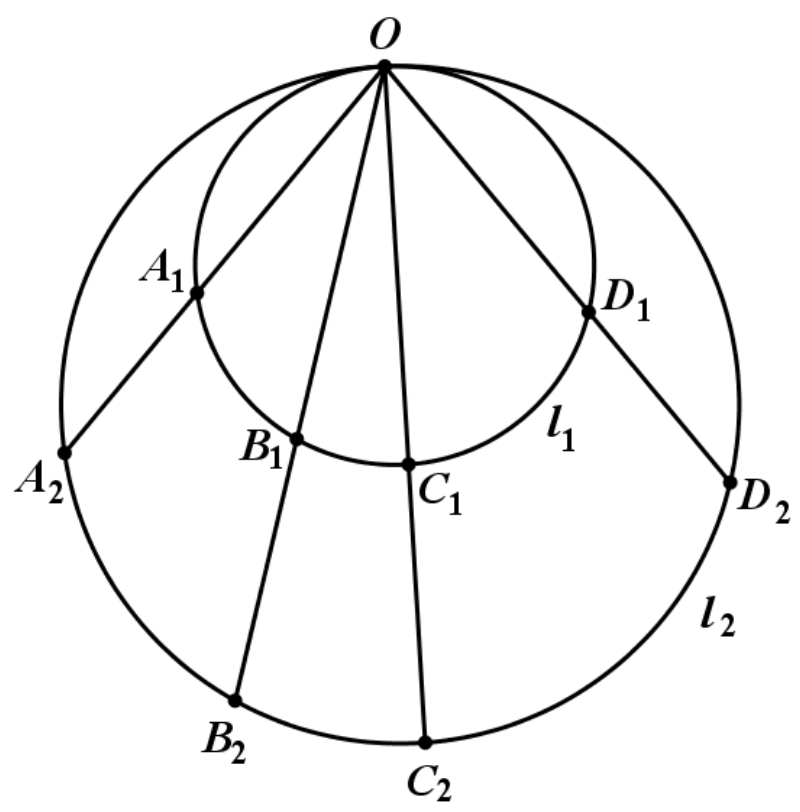

\section{$\underline{\underline{O}}$}

A figura abaixo ilustra a perspectividade $A_{1} B_{1} C_{1} D_{1} \wedge A_{2} B_{2}$ $C_{2} D_{2}$, quando $l_{1}$ é uma circunferência e $l_{2}$ é uma reta. 
Perspectividade entre pontos, quando $l_{1}$ é uma circunferência $l_{2}$ é uma reta

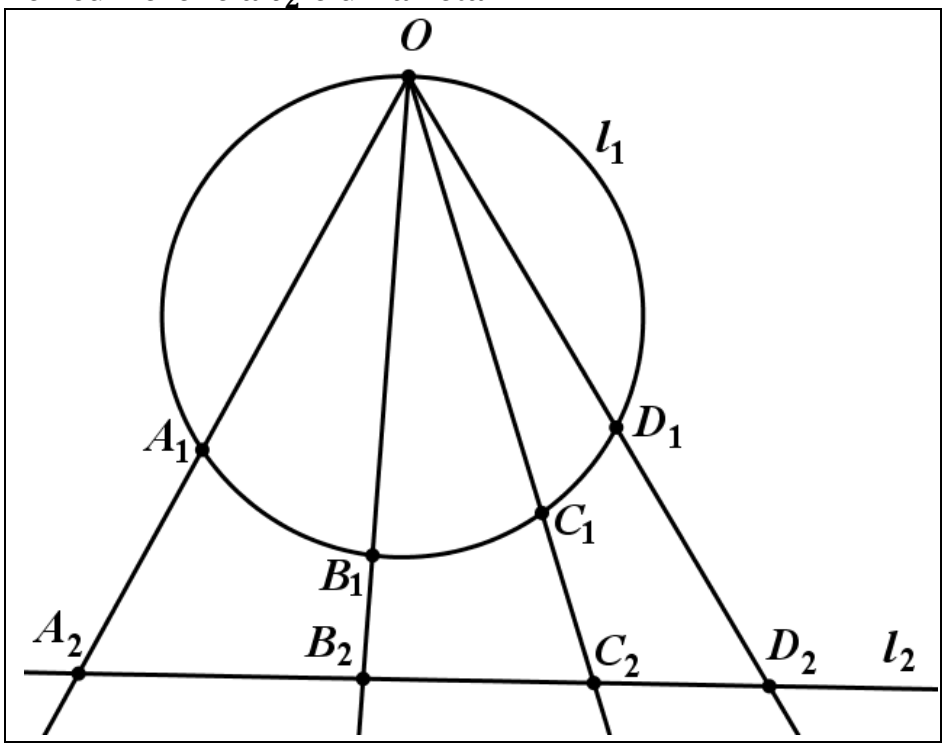

De acordo com as Definições 2 e 3, a perspectividade com relação a um ponto preserva a razão cruzada e, portanto, preserva pontos conjugados harmônicos.

Definição 7. Suponha que $l_{1}$ e $l_{2}$ são tais que cada uma delas é uma reta ou uma circunferência. Uma projetividade (denotada por $\bar{\Lambda}$ ) é qualquer mapeamento de $l_{1}$ a $l_{2}$ que pode ser representado como uma composição finita de perspectividades.

Proposição 5. Existe uma projetividade $A B C D \bar{\wedge} B A D C$ para quaisquer pontos dois a dois distintos $A, B, C$ e $D$ de uma reta.

\section{Demonstração.}

Para mostrar tal fato, vamos elaborar a seguinte construção, conforme a figura abaixo. 


\section{Proposição 5}

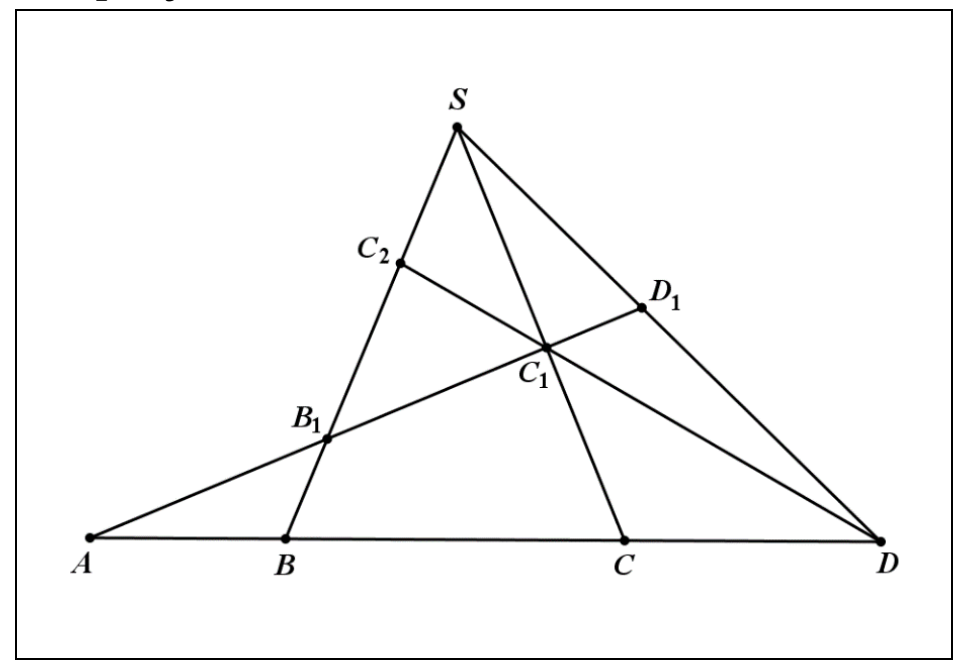

Por um ponto $S$ não incidente com a reta $l=\overleftrightarrow{A B}$ projete $A B C D$ em $A_{1} B_{1} C_{1} D_{1}$ sobre a reta $l_{1}$ incidente a $A$. De $D$ projete $A_{1} B_{1} C_{1} D_{1}$ sobre a reta $\overleftrightarrow{S B}$. Os últimos quatro pontos obtidos serão projetados em $B A D C$ por $C_{1}$, ou seja,

$$
A B C D \stackrel{\underline{S}}{\wedge} \stackrel{\stackrel{D}{\wedge}}{=} \stackrel{\stackrel{C_{1}}{=}}{=} B B_{1} C_{1} D_{1} \stackrel{\wedge}{\wedge} B B_{1} C_{2} S B A C .
$$

Portanto,

$$
A B C D \bar{\wedge} B A D C .
$$

Teorema 1. Suponha que os pontos $A, B, C, D_{1}$ e $D_{2}$ sejam colineares ou concíclicos. Se $R\left(A, B ; C, D_{1}\right)=R\left(A, B ; C, D_{2}\right)$, então $D_{1}=D_{2}$. 


\section{Demonstração.}

Primeiramente vamos demonstrar o caso em que os pontos $A$, $B, C, D_{1}$ e $D_{2}$ são colineares.

Sendo $R\left(A, B ; C, D_{1}\right)=R\left(A, B ; C, D_{2}\right)$, pela Definição 1 , temos

$$
\overline{\overline{C B}} \frac{\overline{D_{1} B}}{\overline{A D_{1}}}=\overline{\overline{C B}} \overline{\overline{C B}} \frac{\overline{D_{2} B}}{\overline{A D_{2}}} .
$$

Isto implica

$$
\overline{\overline{D_{1} B}}=\overline{\overline{D_{2} B}} \overline{\overline{D_{2} A}} .
$$

Visto que os comprimentos são direcionados, temos $\overline{D_{2} B}=$ $\overline{D_{2} D_{1}}+\overline{D_{1} B}$ e $\overline{D_{2} A}=\overline{D_{2} D_{1}}+\overline{D_{1} A}$.

Logo,

o que implica

$$
\frac{\overline{D_{1} B}}{\overline{D_{1} A}}=\frac{\overline{D_{2} D_{1}}+\overline{D_{1} B}}{\overline{D_{2} D_{1}}+\overline{D_{1} A}}
$$

$$
\overline{D_{2} D_{1}}\left(\overline{D_{1} A}-\overline{D_{1} B}\right)=0,
$$

que, por sua vez, implica

$$
\overline{D_{2} D_{1}}=0 \text { ou } \overline{D_{1} A}=\overline{D_{1} B} \text {. }
$$

Se $\overline{D_{2} D_{1}}=0$, então $D_{1}=D_{2}$.

Se $\overline{D_{1} A}=\overline{D_{1} B}$, então $\overline{D_{2} A}=\overline{D_{2} B}$. Daí, $D_{1}=D_{2}$.

Agora, vamos demonstrar o caso em que os pontos $A, B, C, D_{1}$, $D_{2}$ e $O$ são concíclicos.

Sendo $R\left(A, B ; C, D_{1}\right)=R\left(A, B ; C, D_{2}\right)$ pela Definição 3 , temos

$$
R\left(\overleftrightarrow{O A}, \overleftrightarrow{O B} ; \overleftrightarrow{O C}, \overleftrightarrow{O D_{1}}\right)=R\left(\overleftrightarrow{O A}, \overleftrightarrow{O B} ; \overleftrightarrow{O C}, \overleftrightarrow{O D_{2}}\right)
$$

Seja $r$ uma reta que intersecta as retas $\overleftrightarrow{O A}, \overleftrightarrow{O B}, \overleftrightarrow{O C}, \overleftrightarrow{O D_{1}}$ e $\overleftrightarrow{O D_{2}}$ em $A_{1}, B_{1}, C_{1}, D_{3}$ e $D_{4}$, respectivamente.

Logo, pela Definição 2,

$$
R\left(A_{1}, B_{1} ; C_{1}, D_{3}\right)=R\left(A_{1}, B_{1} ; C_{1}, D_{4}\right) .
$$

Com isso, $D_{3}=D_{4}$, ou seja, $\overleftrightarrow{O D_{1}}=\overleftrightarrow{O D_{2}}$.

Portanto, $D_{1}=D_{2}$. 
Corolário 1. Se uma projetividade deixa invariante cada um de três pontos distintos de uma reta (circunferência), então ela deixa invariante todos os pontos da reta (circunferência).

Teorema 2. Se os pontos $A, B, C$ e $D$ são dois a dois distintos, um dos pontos $C, D$ está entre $A$ e $B$ e $R(A, B ; C, D)=$ $R(B, A ; C, D)$, então $H(A, B ; C, D)$.

\section{Demonstração.}

Pela Propriedade 3, temos

$$
R(B, A ; C, D)=R(A, B ; D, C) .
$$

Pela Propriedade 5, temos

$$
R(A, B ; D, C)=\frac{1}{R(A, B ; C, D)} .
$$

Sendo $R(A, B ; C, D)=R(B, A ; C, D)$, então

o que implica

$$
R(A, B ; C, D)=\frac{1}{R(A, B ; C, D)^{\prime}}
$$

$$
R(A, B ; C, D)^{2}=1,
$$

ou seja,

$$
R(A, B ; C, D)=1 \text { ou } R(A, B ; C, D)=-1 .
$$

Pela Propriedade $2, R(A, B ; C, D)<0$. Por isso, $R(A, B ; C, D)=-1$.

Portanto, $H(A, B ; C, D)$.

Proposição 6. Dado um quadrilátero $A B C D$. Sejam, $P=\overleftrightarrow{A B} \cap$ $\overleftrightarrow{C D}, Q=\overleftrightarrow{A D} \cap \overleftrightarrow{B C}, R=\overleftrightarrow{A C} \cap \overleftrightarrow{P Q}$ e $S=\overleftrightarrow{B D} \cap \overleftrightarrow{P Q}$, então $H(P, Q ; R, S)$.

\section{Demonstração.}

Seja $T=\overleftrightarrow{A C} \cap \overleftrightarrow{B D}$. Considere a sequência de perspectividades. 


$$
P Q R S \stackrel{\stackrel{A}{\wedge}}{\wedge} \stackrel{\stackrel{C}{=}}{\wedge} Q P R S .
$$

Como a razão cruzada é preservada por perspectividade, tem-se

$$
R(P, Q ; R, S)=R(Q, P ; R, S) .
$$

Invocando o Teorema 2 , obtemos que $H(P, Q ; R, S)$.

\section{Proposição 6}

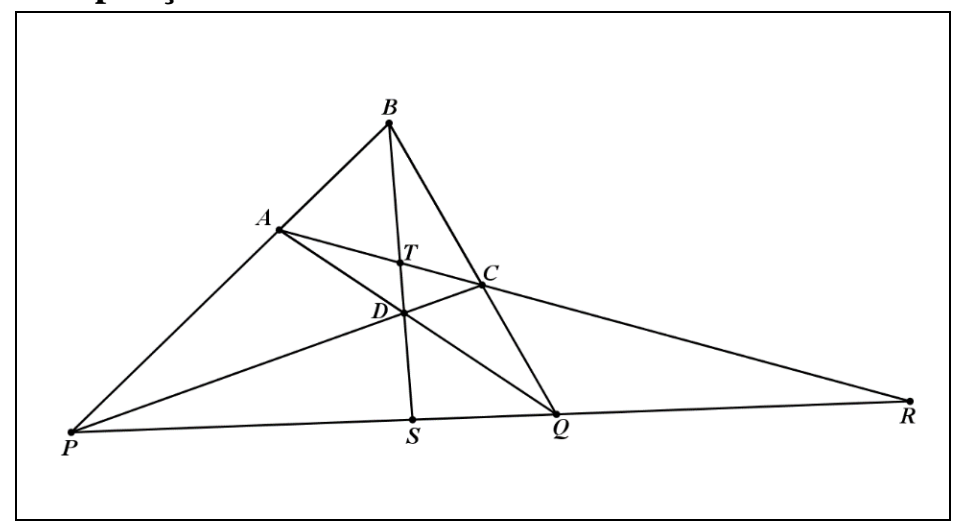

Definição 8. Dizemos que os triângulos $A_{1} B_{1} C_{1}$ e $A_{2} B_{2} C_{2}$ estão em perspectiva em relação a um ponto se as retas $\overleftrightarrow{A_{1} A_{2}}, \overleftrightarrow{B_{1} B_{2}}$ e $\overleftrightarrow{C_{1} C_{2}}$ forem concorrentes. Eles estão em perspectivas em relação a uma reta se os pontos $K=\overleftrightarrow{B_{1} C_{1}} \cap \overleftrightarrow{B_{2} C_{2}}, L=\overleftrightarrow{A_{1} C_{1}} \cap$ $\overleftrightarrow{A_{2} C_{2}}$ e $M=\overleftrightarrow{A_{1} B_{1}} \cap \overleftrightarrow{A_{2} B_{2}}$ forem colineares.

Teorema de Desargues. Dois triângulos estão em perspectivas em relação a um ponto se, e somente se, eles estão em perspectiva em relação a uma reta.

\section{Demonstração.}

Sejam $P Q R$ e $P_{1} Q_{1} R_{1}$ dois triângulos. Temos primeiro que mostrar que, se as retas $\overleftrightarrow{P P_{1}}, \overleftrightarrow{Q Q_{1}}$ e $\overleftrightarrow{R R_{1}}$ são concorrentes em $O$, então os pontos 


$$
A=\overleftrightarrow{Q R} \cap \overleftrightarrow{Q_{1} R_{1}}, B=\overleftrightarrow{R P} \cap \overleftrightarrow{R_{1} P_{1}} \text { e } C=\overleftrightarrow{P Q} \cap \overleftrightarrow{P_{1} Q_{1}}
$$

são colineares.

Repare que o teorema é trivial quando os dois triângulos estão em planos distintos. Neste caso, os pontos $A, B$ e $C$ estão ambos nos planos $\alpha=P Q R$ e $\beta=P_{1} Q_{1} R_{1}$. Portanto, estão sobre a reta $\alpha \cap \beta$. Como ilustra a figura abaixo.

\section{Demonstração do Teorema de Desargues}

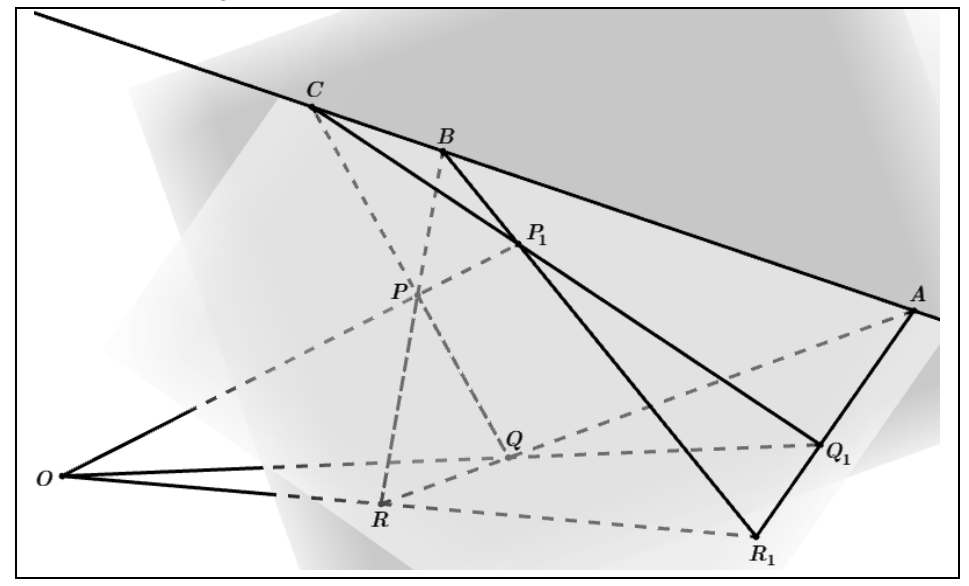

Caso os triângulos estejam em um mesmo plano, tomamos dois pontos $S$ e $S_{1}$ numa reta qualquer incidente no ponto $O$ e fora do plano $P Q R$. Assim, as retas $\overleftrightarrow{P P_{1}}, \overleftrightarrow{Q Q_{1}}, \overleftrightarrow{R R_{1}}$ e $\overleftrightarrow{S S_{1}}$ passam todas por $O$. Como $P, P_{1}, S$ e $S_{1}$ pertencem ao plano $O P S$, segue que $\overleftrightarrow{P S}$ e $\overleftrightarrow{P_{1} R_{1}}$ se intersectam em um ponto $P_{2}$. Analogamente, determinamos os pontos $Q_{2}=\overleftrightarrow{Q S} \cap \overleftrightarrow{Q_{1} S_{1}}$ e $R_{2}=\overleftrightarrow{R S} \cap \overleftrightarrow{R_{1} S_{1}}$. Aplicando a parte óbvia do teorema para os triângulos $Q R S$, $Q_{1} R_{1} S_{1}$, que estão em planos distintos, temos que os pontos

$$
R_{2}=\overleftrightarrow{R S} \cap \overleftrightarrow{R_{1} S_{1}}, Q_{2}=\overleftrightarrow{S Q} \cap \overleftrightarrow{S_{1} Q_{1}} \text { e } A=\overleftrightarrow{Q R} \cap \overleftrightarrow{Q_{1} R_{1}}
$$

são colineares. Portanto, $A \in \overleftrightarrow{Q_{1} R_{1}}$. Analogamente, temos $B \in \overleftrightarrow{P_{1} R_{1}}$ e $C \in \overleftrightarrow{P_{1} Q_{1}}$. Logo, os três pontos $A, B$ e $C$ estão sobre 
a reta $\alpha \cap \beta$, onde $\alpha$ é o plano que contém os pontos $P, Q$ e $R$, e $\beta$ é o plano que contém os pontos $P_{2}, Q_{2}$ e $R_{2}$.

\section{Demonstração do Teorema de Desargues}

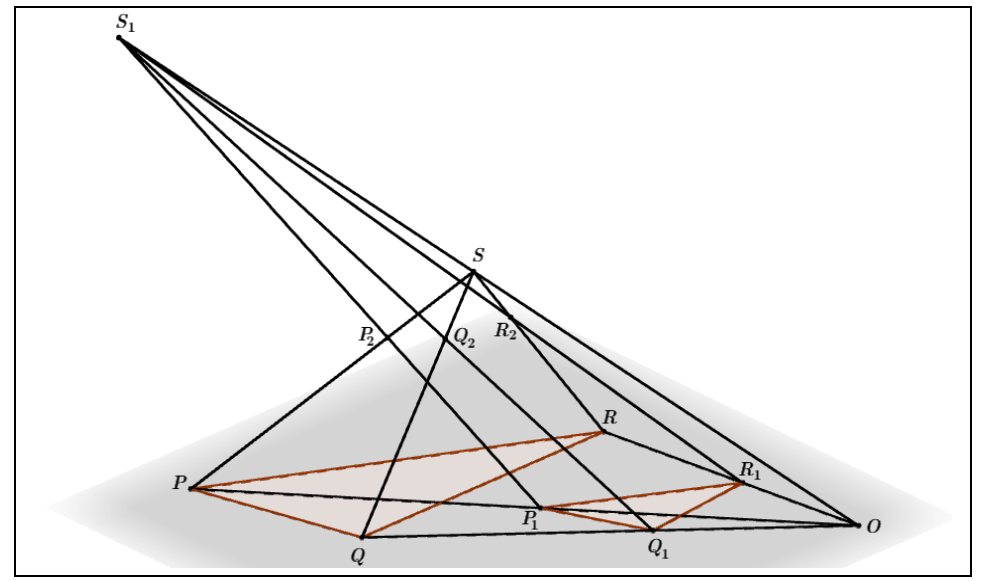

Suponha agora que os triângulos $P Q R$ e $P_{1} Q_{1} R_{1}$ estão em perspectiva em relação a uma reta. Temos que mostrar que as retas $\overleftrightarrow{P P_{1}}, \overleftrightarrow{Q Q_{1}}$ e $\overleftrightarrow{R R_{1}}$ são concorrentes em $O$. Com isso, os pontos $\quad A=\overleftrightarrow{Q R} \cap \overleftrightarrow{Q_{1} R_{1}}, B=\overleftrightarrow{R P} \cap \overleftrightarrow{R_{1} P_{1}}$ e $C=\overleftrightarrow{Q P} \cap \overleftrightarrow{Q_{1} P_{1}}$ são colineares. Por esse motivo, os triângulos $P P_{1} B$ e $Q Q_{1} A$ estão em perspectiva em relação ao ponto $C$.

Portanto, $O=\overleftrightarrow{P P_{1}} \cap \overleftrightarrow{Q Q_{1}}, R_{1}=\overleftrightarrow{B P_{1}} \cap \overleftrightarrow{A Q_{1}}$ e $R=\overleftrightarrow{B P} \cap \overleftrightarrow{A Q}$ são colineares. Daí, $O=\overleftrightarrow{R R_{1}} \cap \overleftrightarrow{P P_{1}} \cap \overleftrightarrow{Q Q_{1}}$, o que mostra que os triângulos $P Q R$ e $P_{1} Q_{1} R_{1}$ estão em perspectiva em relação ao ponto $O$.

Teorema de Monge. Se $C_{1}, C_{2}$ e $C_{3}$ são círculos, então a interseção das retas tangentes externas (centro externo de similaridade) dos círculos $C_{1}$ e $C_{2}, C_{1}$ e $C_{3}$, e $C_{2}$ e $C_{3}$ são colineares. 


\section{Demonstração.}

Sejam $O_{1}, O_{2}$ e $O_{3}$ os centros dos círculos $C_{1}, C_{2}$ e $C_{3}$, respectivamente. Sendo $A_{1}$ a interseção das retas tangentes aos pares de círculos $C_{1}, C_{2}$ e $C_{1}, C_{3}, A_{2}$ a interseção das retas tangentes aos pares de círculos $C_{2}, C_{1}$ e $C_{2}, C_{3}$ e $A_{3}$ a interseção das retas tangentes aos pares de círculos $C_{3}, C_{1}$ e $C_{3}, C_{2}$, onde todas as retas tangentes mencionadas acima são disjuntas ao triângulo $O_{1} O_{2} O_{3}$. Com isso, as retas $\overleftrightarrow{A_{1} O_{1}}, \overleftrightarrow{A_{2} O_{2}}$ e $\overleftrightarrow{A_{3} O_{3}}$ são as bissetrizes internas do triângulo $A_{1} A_{2} A_{3}$. Portanto, os triângulos $A_{1} A_{2} A_{3}$ e $O_{1} O_{2} O_{3}$ estão em perspectivas em relação ao ponto $I$, incentro do triângulo $A_{1} A_{2} A_{3}$. Pelo Teorema de Desargues, os exincentros $\quad X=\overleftrightarrow{A_{1} A_{2}} \cap \overleftrightarrow{O_{1} O_{2}}, \quad Y=\overleftrightarrow{A_{1} A_{3}} \cap \overleftrightarrow{O_{1} O_{3}} \quad$ e $\quad Z=$ $\overleftrightarrow{\mathrm{A}_{2} \mathrm{~A}_{3}} \cap \overleftrightarrow{\mathrm{O}_{2} \mathrm{O}_{3}}$ são colineares.

\section{Demonstração do Teorema de Monge}

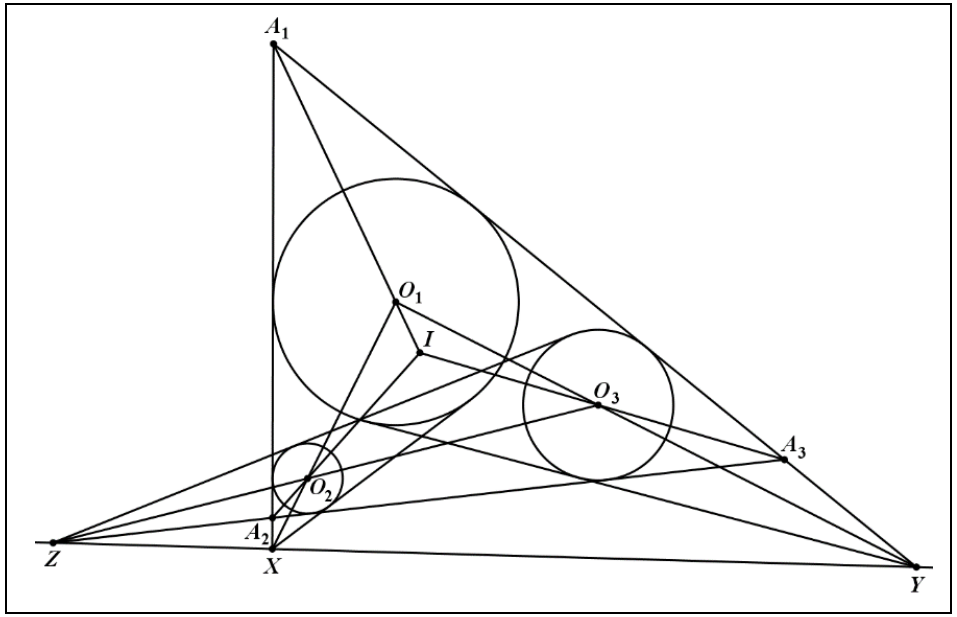

Teorema de Monge e D'Alembert. São dados três círculos $C_{1}, C_{2}$ e $C_{3}$. Se as retas tangentes internas comuns aos círculos $C_{1}$ e $C_{2}$ se intersectam em $X$ (centro interno de similaridade), as retas tangentes internas comuns aos círculos $C_{1}$ e $C_{3}$ se 
intersectam em $Y$ (centro interno de similaridade) e as retas tangentes externas comuns aos círculos $C_{2}$ e $C_{3}$ se intersectam em $Z$ (centro externo de similaridade), então os pontos $X, Y$ e $Z$ são colineares.

\section{Demonstração.}

Sejam $O_{1}, O_{2}$ e $O_{3}$ os centros dos círculos $C_{1}, C_{2}$ e $C_{3}$, respectivamente. A reta $\overleftrightarrow{X Y}$ divide o plano em dois semiplanos, um contém o ponto $O_{1}$ e o outro contêm os pontos $O_{2}$ e $O_{3}$. Sejam $A_{1}$ um ponto pertencente ao semiplano que contém o ponto $O_{1}$ e interno ao triângulo $O_{1} O_{2} O_{3}$, obtido pela interseção das retas tangentes aos pares de círculos $C_{1}, C_{2}$ e $C_{1}, C_{3}, A_{2}$ a interseção da reta $\overleftrightarrow{A X}$ com a reta tangente aos círculos $C_{2}, C_{3}$ e disjunta ao triângulo $O_{1} O_{2} O_{3}$, e $A_{3}$ a interseção das retas $\overleftrightarrow{A Y}$ e $\overleftrightarrow{B Z}$. Com isso, as retas $\overleftrightarrow{A_{1} O_{1}}, \overleftrightarrow{A_{2} O_{2}}$ e $\overleftrightarrow{A_{3} O_{3}}$ são as bissetrizes internas do triângulo $A_{1} A_{2} A_{3}$. Portanto, os triângulos $A_{1} A_{2} A_{3}$ e $\mathrm{O}_{1} \mathrm{O}_{2} \mathrm{O}_{3}$ estão em perspectivas em relação ao ponto $I$, incentro do triângulo $A_{1} A_{2} A_{3}$. Pelo Teorema de Desargues, os pontos $X=\overleftrightarrow{A_{1} A_{2}} \cap \overleftrightarrow{O_{1} O_{2}}, Y=\overleftrightarrow{A_{1} A_{3}} \cap \overleftrightarrow{O_{1} O_{3}}$ e $Z=\overleftrightarrow{A_{2} A_{3}} \cap \overleftrightarrow{O_{2} O_{3}}$ são colineares. 


\section{Demonstração do Teorema de Monge e D'Alembert}

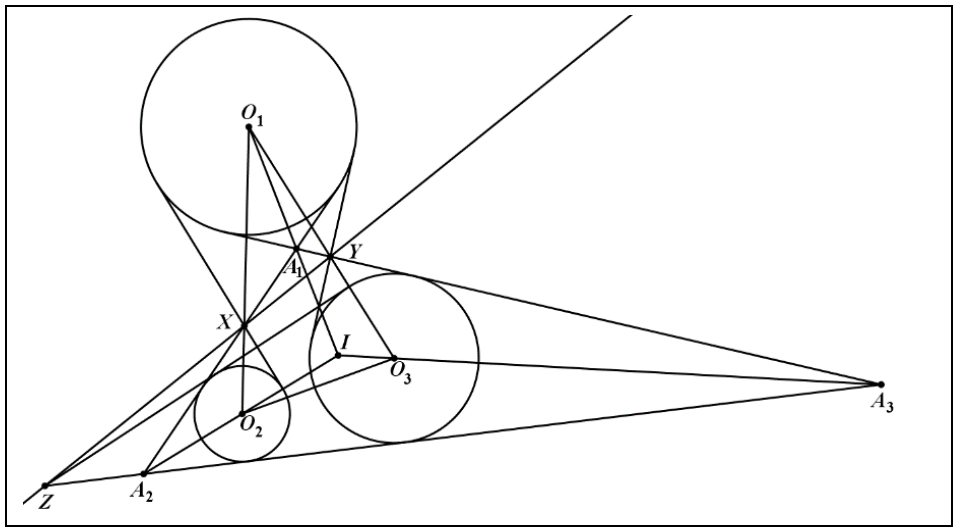

Teorema de Pappus. Se os pontos $A_{1}, A_{2}$ e $A_{3}$ pertencem à reta $a$, e os pontos $B_{1}, B_{2}$ e $B_{3}$ pertencem à reta $b$, então os pontos $C_{3}=\overleftrightarrow{A_{1} B_{2}} \cap \overleftrightarrow{A_{2} B_{1}}, C_{2}=\overleftrightarrow{A_{1} B_{3}} \cap \overleftrightarrow{A_{3} B_{1}}$ e $C_{1}=\overleftrightarrow{A_{2} B_{3}} \cap$ $\overleftrightarrow{A_{3} B_{2}}$ são colineares.

\section{Demonstração.}

Considere os pontos $C_{4}, D, E$ e $F$ definidos por $C_{4}=\overleftrightarrow{C_{1} C_{3}} \cap$ $\overleftrightarrow{A_{3} B_{1}}, \quad D=\overleftrightarrow{A_{1} B_{2}} \cap \overleftrightarrow{A_{3} B_{1}}, \quad E=\overleftrightarrow{A_{2} B_{1}} \cap \overleftrightarrow{A_{3} B_{2}}$ e $\quad F=a \cap b$. Vamos provar que $C_{4}=C_{2}$.

Considere a sequência de perspectividades:

$$
A_{3} B_{1} D C_{2} \stackrel{A_{1}}{=} F B_{1} B_{2} B_{3} \stackrel{A_{2}}{\wedge} A_{3} E B_{2} C_{1} \stackrel{C_{3}}{\wedge} A_{3} B_{1} D C_{4} .
$$

Logo,

$$
A_{3} B_{1} D C_{2} \bar{\wedge} A_{3} B_{1} D C_{4} .
$$

Pelo Corolário $1, C_{4}=C_{2}$. Como, por definição, $C_{1}, C_{3}$ e $C_{4}$ são colineares, concluímos que $C_{1}, C_{2}$ e $C_{3}$ são colineares. 


\section{Demonstração do Teorema de Pappus}

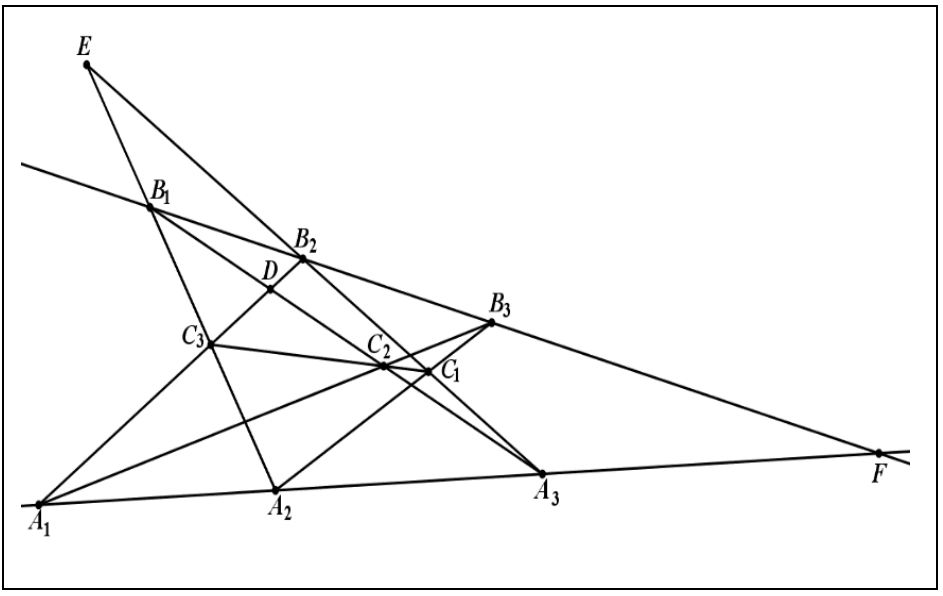

Teorema de Pascal. Sejam $A_{1}, A_{2}, A_{3}, B_{1}, B_{2}$ e $B_{3}$ pontos concíclicos. Se $C_{3}=\overleftrightarrow{A_{1} B_{2}} \cap \overleftrightarrow{A_{2} B_{1}}, \quad C_{2}=\overleftrightarrow{A_{1} B_{3}} \cap \overleftrightarrow{A_{3} B_{1}} \quad \mathrm{e}$ $C_{1}=\overleftrightarrow{A_{2} B_{3}} \cap \overleftrightarrow{A_{3} B_{2}}$, então os pontos $C_{1}, C_{2}$ e $C_{3}$ são colineares.

\section{Demonstração.}

Considere os ponto $C_{4}, D$ e $E$ definido por $C_{4}=\overleftrightarrow{C_{1} C_{3}} \cap \overleftrightarrow{A_{3} B_{1}}$, $D=\overleftrightarrow{A_{1} B_{2}} \cap \overleftrightarrow{A_{3} B_{1}}$ e $E=\overleftrightarrow{A_{2} B_{1}} \cap \overleftrightarrow{A_{3} B_{2}}$. Vamos provar que $C_{4}=C_{2}$.

Considere a sequência de perspectividades:

$$
A_{3} B_{1} D C_{2} \stackrel{A_{1}}{\wedge} A_{3} B_{1} B_{2} B_{3} \stackrel{A_{2}}{\stackrel{ }{\wedge}} A_{3} E B_{2} C \stackrel{C_{3}}{\stackrel{ }{\wedge}}{ }_{1} A_{3} B_{1} D C_{4} .
$$

Logo,

$$
A_{3} B_{1} D C_{2} \pi A_{3} B_{1} D C_{4} .
$$

Pelo Corolário $1, C_{4}=C_{2}$. Como, por definição, $C_{1}, C_{3}$ e $C_{4}$ são colineares, concluímos que $C_{1}, C_{2}$ e $C_{3}$ são colineares. 


\section{Demonstração do Teorema de Pascal}

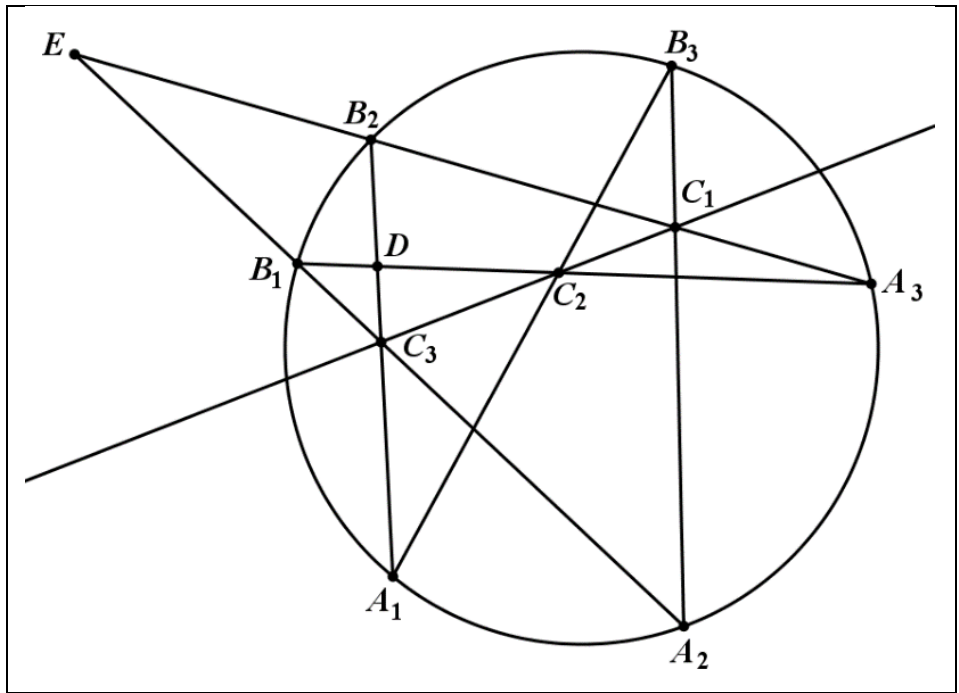

Definição 9. Dado uma circunferência $C(O, r)$. Seja $A^{*}$ a imagem do ponto $A \neq O$ pela inversão em relação à circunferência $C$. A reta $a$ passando por $A^{*}$ e perpendicular a $\overleftrightarrow{O A}$ é chamada a polar de $A$ em relação a $C$. Inversamente, o ponto $A$ é chamado o pólo da reta $a$ em relação a $C$.

Teorema 3. Sejam $A$ e $B$ dois pontos do plano, com $a$ e $b$ suas respectivas polares em relação a uma dada circunferência $C$. Tem-se que $A \in b$ se, e somente se, $B \in a$.

\section{Demonstração.}

Considere um ponto $B \in a$. Seja $B_{1} \in \overrightarrow{O B}$ tal que $\overleftrightarrow{A B_{1}} \perp \overleftrightarrow{O B}$. Os triângulos $O A B_{1}$ e $O B A^{*}$ são retângulos e têm o ângulo $\angle A O B_{1}$ e $\angle B O A^{*}$ em comum; logo, são semelhantes. Com isso,

o que implica

$$
\overline{\overline{O B}}=\frac{\overline{O B_{1}}}{\overline{O A^{*}}}
$$




$$
\overline{O B} \cdot \overline{O B_{1}}=\overline{O A} \cdot \overline{O A^{*}}=r^{2} .
$$

Portanto, $B_{1}$ é o inverso de $B$, ou seja, $B_{1}=B^{*}$. Daí, $\overleftrightarrow{A B_{1}}=b$ e $A \in b$.

Analogamente, se $A \in b$, então $B \in a$.

Definição 10. Os pontos $A$ e $B$ são chamados conjugados em relação à circunferência $C$, se um deles estiver na polar do outro.

Teorema 4. Se a reta determinada por dois pontos conjugados $A$ e $B$ intersecta a circunferência $\Gamma$ em $C$ e $D$, então $H(A, B ; C, D)$. Reciprocamente, se $H(A, B ; C, D)$, onde $C$ e $D \in \Gamma$, então $A$ e $B$ são conjugados em relação à circunferência $\Gamma$.

\section{Demonstração.}

Sejam $C_{1}$ e $D_{1}$ pontos de interseção da reta $\overleftrightarrow{O A}$ com a circunferência $\Gamma$, onde $O$ é o centro da circunferência $\Gamma$. Dado que a inversão preserva a razão cruzada, obtemos

$$
R\left(C_{1}, D_{1} ; A, A^{*}\right)=R\left(C_{1}, D_{1} ; A^{*}, A\right) .
$$

Pelo Teorema 2,

$$
H\left(C_{1}, D_{1} ; A, A^{*}\right)
$$

Seja $p$ a reta que contém $A$ e intersecta a circunferência $\Gamma$ nos pontos $C$ e $D$. Sejam $E=\overleftrightarrow{C C_{1}} \cap \overleftrightarrow{D D_{1}}$ e $F=\overleftrightarrow{C D_{1}} \cap \overleftrightarrow{C_{1} D}$. Como $C_{1} D_{1}$ é o diâmetro da circunferência $\Gamma$, temos

$$
\overleftrightarrow{C_{1} F} \perp \overleftrightarrow{D_{1} E} \text { e } \overleftrightarrow{D_{1} F} \perp \overleftrightarrow{C_{1} E}
$$

Portanto, o ponto $F$ é o ortocentro do triângulo $C_{1} D_{1} E$.

Sejam $B=\overleftrightarrow{E F} \cap \overleftrightarrow{C D}$ e $A_{1}=\overleftrightarrow{E F} \cap \overleftrightarrow{C_{1} D_{1}}$. Como

$$
C_{1} D_{1} A A_{1} \stackrel{\underline{\underline{E}}}{\wedge} \mathrm{CDAB} \stackrel{\underline{F}}{\wedge} D_{1} C_{1} A A_{1},
$$


tem-se

$$
C_{1} D_{1} A A_{1} \bar{\wedge} D_{1} C_{1} A A_{1}
$$

o que implica

$$
R\left(C_{1}, D_{1} ; A, A_{1}\right)=R\left(D_{1}, C_{1} ; A, A_{1}\right) .
$$

Pelo Teorema $2, H\left(C_{1}, D_{1} ; A, A_{1}\right)$. Como perspectividade preserva pontos conjugados harmônicos, $H(C, D ; A, B)$.

De $H\left(C_{1}, D_{1} ; A, A^{*}\right)$ e $H\left(C_{1}, D_{1} ; A, A_{1}\right)$ obtemos, $A^{*}=A_{1}$; portanto, $A^{*} \in \overleftrightarrow{E F}$. No entanto, $\overleftrightarrow{E F} \perp \overleftrightarrow{C_{1} D_{1}}$ e a reta $\overleftrightarrow{E F}=a$ é a polar do ponto $A$. Com isso, $B=\overleftrightarrow{E F} \cap \overleftrightarrow{C D} \in$ a. Pelo Teorema 3, $A \in b$, onde $b$ é a polar do ponto $B$.

Portanto, $A$ e $B$ são pontos conjugados em relação à circunferência $\Gamma$.

Para o ponto $B$ pertencente à polar do ponto $A$, temos

$$
C_{1} D_{1} A A^{*} \stackrel{\underline{\underline{E}}}{\wedge} \mathrm{CDAB} .
$$

Sendo $H\left(C_{1}, D_{1} ; A, A^{*}\right)$, conclui-se que $H(C, D ; A, B)$. Portanto,

$$
H(A, B ; C, D)
$$




\section{Demonstração do Teorema 4}

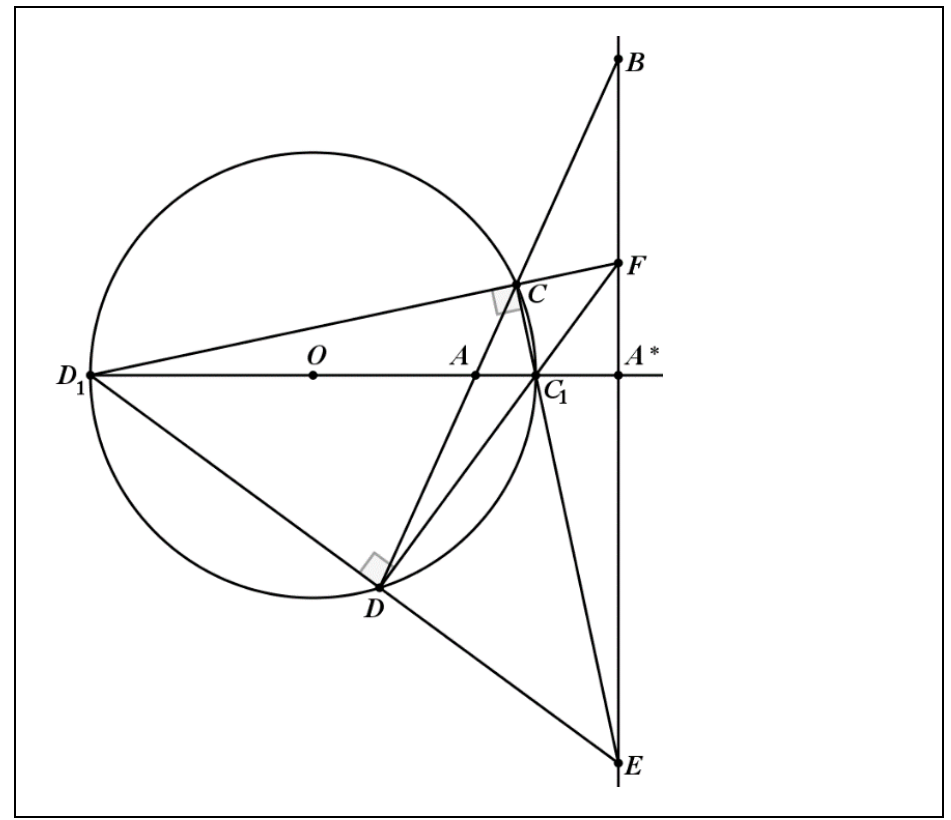

Teorema de Brianchon. Suponha que o hexágono $A_{1} A_{2} A_{3} A_{4} A_{5} A_{6}$ esteja circunscrito à circunferência $C(O, r)$. Então as retas $\overleftrightarrow{A_{1} A_{4}}, \overleftrightarrow{A_{2} A_{5}}$ e $\overleftrightarrow{A_{3} A_{6}}$ são concorrentes. 


\section{Teorema de Brianchon}

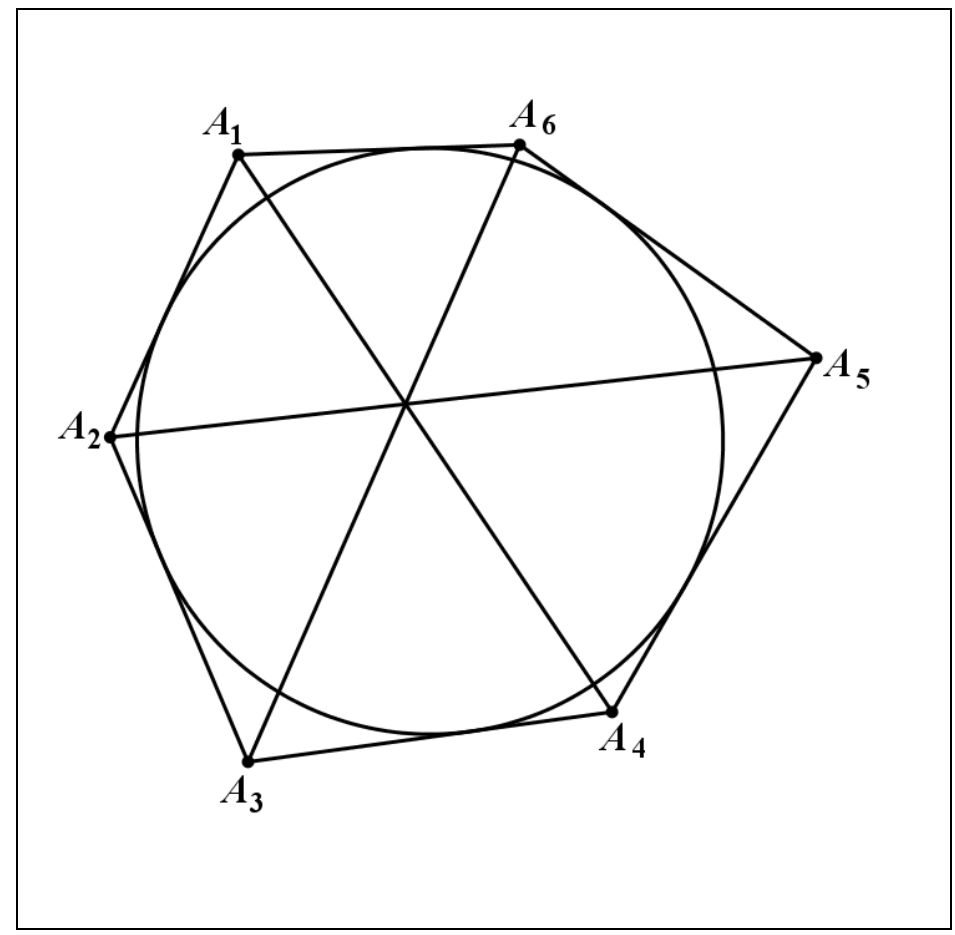

\section{Demonstração.}

Usaremos a convenção em que os pontos serão indicados por letras maiúsculas, e suas polares com as correspondentes letras minúsculas.

Denotando por $M_{i}, i=1,2,3,4,5,6$, os pontos de tangência de $A_{i} A_{i+1}$ com a circunferência $C$, temos $m_{i}=\overleftrightarrow{A_{l} A_{l+1}}$, onde $A_{7}=A_{1}$. Logo, pelo Teorema $3, M_{i} \in a_{i}$ e $M_{i} \in a_{i+1}$. Portanto, $a_{i}=\overleftrightarrow{M_{l-1} M_{l}}$, onde $M_{0}=M_{6}$.

Seja $b_{j}=\overleftrightarrow{A_{\jmath} A_{\jmath+3}}, j=1,2,3$. Então, pelo Teorema $3, B_{j}=a_{j} \cap$ $a_{j+3}=\overleftrightarrow{M_{J-1} M_{J}} \cap \overleftrightarrow{M_{J+2} M_{J+3}}$.

Temos que provar que existe um ponto $P$ tal que $P \in b_{1}, b_{2}, b_{3}$, ou analogamente, que existe uma reta $p$ tal que $B_{1}, B_{2}, B_{3} \in p$. 
Por isso, basta provar que os pontos $B_{1}, B_{2}$ e $B_{3}$ são colineares. No entanto, aplicando o Teorema de Pascal a

$$
M_{1} M_{3} M_{5} M_{4} M_{6} M_{2} \text {, }
$$

concluímos que os pontos $B_{1}=\overleftrightarrow{M_{1} M_{6}} \cap \overleftrightarrow{M_{3} M_{4}}, B_{2}=\overleftrightarrow{M_{1} M_{2}} \cap$ $\overleftrightarrow{M_{4} M_{5}}$ e $B_{3}=\overleftrightarrow{M_{2} M_{3}} \cap \overleftrightarrow{M_{5} M_{6}}$ são colineares.

Teorema de Brocard. O quadrilátero $A B C D$ está inscrito na circunferência $C(O, r)$. Se $E=\overleftrightarrow{A B} \cap \overleftrightarrow{C D}, \quad F=\overleftrightarrow{A D} \cap \overleftrightarrow{B C}$ e $G=\overleftrightarrow{A C} \cap \overleftrightarrow{B D}$, então $O$ é o ortocentro do triângulo $E F G$.

\section{Demonstração.}

Vamos provar que $\overleftrightarrow{E G}$ é a polar do ponto $F$. Sejam $X=\overleftrightarrow{E G} \cap$ $\overleftrightarrow{B C}$ e $Y=\overleftrightarrow{E G} \cap \overleftrightarrow{A D}$. Então,

$$
A D Y F \stackrel{\underline{\underline{E}}}{\wedge} B C X F \stackrel{\underline{\underline{G}}}{\wedge} D A Y F
$$

Pelo Teorema 2, $H(A, D ; Y, F)$. Como perspectividade preserva a relação harmônica, com isso, $H(B, C ; X, F)$. De acordo com o Teorema 4, os pontos $X$ e $Y$ estão na reta polar do ponto $F$; portanto, $\overleftrightarrow{E G}$ é a polar do ponto $F$.

Como $\overleftrightarrow{E G}$ é a polar de $F$, temos $\overleftrightarrow{E G} \perp \overleftrightarrow{O F}$. Analogamente, obtemos $\overleftrightarrow{F G} \perp \overleftrightarrow{O E}$

Portanto, $O$ é o ortocentro do triângulo $E F G$. 
Demonstração do Teorema de Brocard

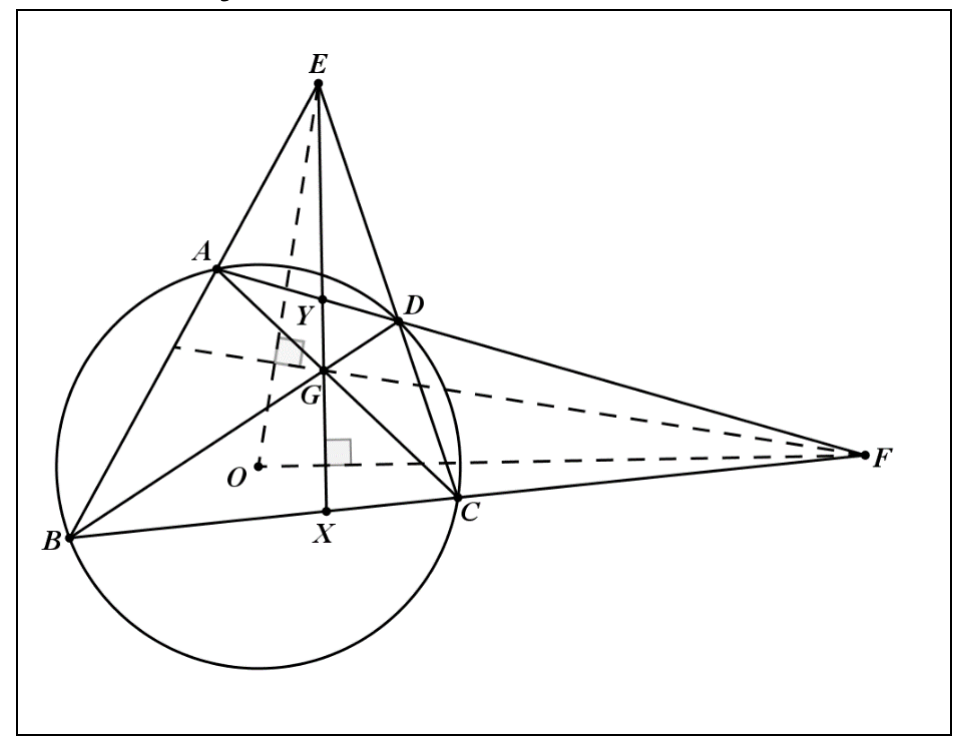




\section{SOLUÇÃO DOS PROBLEMAS}

1. Dado o triângulo $A B C$ e um ponto $M$ no segmento $B C$, seja $N$ o ponto da reta $\overleftrightarrow{B C}$ tal que $\angle M A N=90^{\circ}$. Prove que $H(B, C ; M, N)$ se, e somente se, $A M$ é a bissetriz do ângulo $\angle B A C$.

\section{Solução.}

Sejam $\alpha=\angle B A C, \beta=\angle C B A, \theta=\angle A C B$ e $\varphi=$ $\angle B A M$, como ilustra a figura abaixo.

\section{Problema 1}

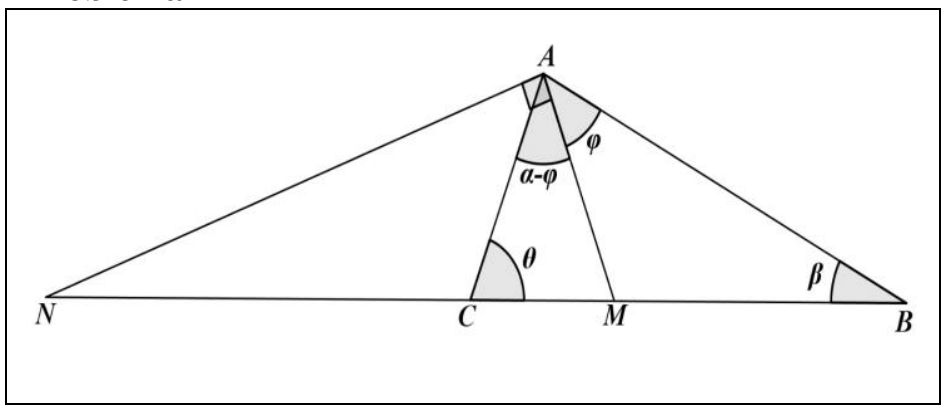

Aplicando a lei dos senos nos triângulos $A B M$ e $A C M$, tem-se

$$
\frac{\overline{B M}}{\operatorname{sen} \varphi}=\frac{\overline{A M}}{\operatorname{sen} \beta} \Rightarrow \frac{\overline{B M}}{\overline{A M}}=\frac{\operatorname{sen} \varphi}{\operatorname{sen} \beta}
$$

e

$$
\frac{\overline{A M}}{\operatorname{sen} \theta}=\frac{\overline{M C}}{\operatorname{sen}(\alpha-\varphi)} \Rightarrow \frac{\overline{A M}}{\overline{M C}}=\frac{\operatorname{sen} \theta}{\operatorname{sen}(\alpha-\varphi)}
$$

Multiplicando (1) por (2), obtemos

$$
\frac{\overline{B M}}{\overline{M C}}=\frac{\overline{B M}}{\overline{A M}} \frac{\overline{A M}}{\overline{M C}}
$$




$$
=\frac{\operatorname{sen} \varphi}{\operatorname{sen} \beta} \frac{\operatorname{sen} \theta}{\operatorname{sen}(\alpha-\varphi)} .
$$

Analogamente, aplicando a lei dos senos nos triângulos $A B N$ e $A C N$, tem-se

$$
\frac{\overline{B N}}{\operatorname{sen}\left(\varphi+90^{\circ}\right)}=\frac{\overline{A N}}{\operatorname{sen} \beta} \Rightarrow \frac{\overline{A N}}{\overline{B N}} \frac{\operatorname{sen} \beta}{\operatorname{sen}\left(\varphi+90^{\circ}\right)}
$$

e

$$
\begin{gathered}
\frac{\overline{A N}}{\operatorname{sen}\left(180^{\circ}-\theta\right)}=\frac{\overline{N C}}{\operatorname{sen}\left(90^{\circ}+\varphi-\alpha\right)} \Rightarrow \\
\Rightarrow \frac{\overline{N C}}{\overline{A N}}=\frac{\operatorname{sen}\left(90^{\circ}+\varphi-\alpha\right)}{\operatorname{sen}\left(180^{\circ}-\theta\right)} .
\end{gathered}
$$

Multiplicando (4) por (5), obtemos

$$
\begin{aligned}
\frac{\overline{N C}}{\overline{B N}}=\frac{\overline{N C} \overline{A N}}{\overline{A N}} \frac{\overline{B N}}{} \\
=\frac{\operatorname{sen}\left(90^{\circ}+\varphi-\alpha\right)}{\operatorname{sen}\left(180^{\circ}-\theta\right)} \frac{\operatorname{sen} \beta}{\operatorname{sen}\left(\varphi+90^{\circ}\right)} .
\end{aligned}
$$

Usando a definição de razão cruzada e as identidades (3) e (6), obtemos

$$
\begin{aligned}
& R(B, C ; M, N)=\frac{\overline{B M}}{\overline{M C}} \overline{\overline{B C}} \\
& =\frac{\operatorname{sen} \varphi}{\operatorname{sen} \beta} \frac{\operatorname{sen} \theta}{\operatorname{sen}(\alpha-\varphi)} \frac{\operatorname{sen}\left(90^{\circ}+\varphi-\alpha\right)}{\operatorname{sen}\left(180^{\circ}-\theta\right)} \frac{\operatorname{sen} \beta}{\operatorname{sen}\left(\varphi+90^{\circ}\right)} \\
& =\frac{\operatorname{sen} \varphi \cdot \operatorname{sen} \theta \cdot \cos (\varphi-\alpha)}{\operatorname{sen}(\alpha-\varphi) \cdot \cos \varphi \cdot \operatorname{sen} \theta} \\
& =\frac{\tan \varphi}{\tan (\alpha-\varphi)} .
\end{aligned}
$$


Sendo $\quad B \neq C$ e $M \neq N, \quad$ a relação $|R(B, C ; M, N)|=$ 1 implica que $R(B, C ; M, N)=-1$, pois as semirretas $\overrightarrow{B M}$, $\overrightarrow{M C}$ e $\overrightarrow{B N}$ têm o mesmo sentido e a semirreta $\overrightarrow{N C}$ tem sentido oposto.

Portanto, $H(B, C ; M, N) \Leftrightarrow R(B, C ; M, N)=-1$

$$
\begin{aligned}
& \Leftrightarrow|R(B, C ; M, N)|=1 \\
& \Leftrightarrow \tan \varphi=\tan (\alpha-\varphi) \\
& \Leftrightarrow \alpha=2 \varphi \\
& \Leftrightarrow A M \text { é a bissetriz do ângulo } \angle B A C .
\end{aligned}
$$

2. Sejam $A, B$ e $C$ os pontos diagonais do quadrilátero $P Q R S$, ou equivalentemente, $\quad A=\overleftrightarrow{P Q} \cap \overleftrightarrow{R S}, \quad B=\overleftrightarrow{Q R} \cap \overleftrightarrow{S P} \mathrm{e}$ $C=\overleftrightarrow{P R} \cap \overleftrightarrow{Q S}$. Se apenas os pontos $A, B, C$ e $S$ são dados, usando apenas régua, construa os pontos $P, Q$ e $R$.

\section{Solução.}

Trace as retas $\overleftrightarrow{A S}$ e $\overleftrightarrow{B C}$ e marque o ponto de interseção dessas retas. Chamemos esse ponto de $D$. Temos que $Q B P C$ é um quadrilátero tal que $R=\overleftrightarrow{Q B} \cap \overleftrightarrow{P C}, \quad S=\overleftrightarrow{Q C} \cap \overleftrightarrow{B P}, \quad A=$ $\overleftrightarrow{Q P} \cap \overleftrightarrow{R S}$ e $D=\overleftrightarrow{B C} \cap \overleftrightarrow{R S}$. Conclui-se então, pela Proposição 6 , que $H(D, A ; S, R)$. Como $R(D, A ; S, R)=R(A, D ; S, R) \mathrm{e}$ são conhecidas as posições dos pontos $D, A$ e $S$, podemos construir $R$ da seguinte forma: escolha um ponto $K$ não pertencente à reta $\overleftrightarrow{A D}$. Trace a reta $\overleftrightarrow{A K}$ e marque um ponto $L$ na reta $\overleftrightarrow{A K}$, com $L$ diferente de $A$ e $K$. Em seguida, construa as retas $\overleftrightarrow{L D}$ e $\overleftrightarrow{K S}$ e marque o ponto de interseção da reta $\overleftrightarrow{L D}$ com a reta $\overleftrightarrow{K S}$. Chamemos esse ponto de $M$. Trace as retas $\overleftrightarrow{A M}$ e $\overleftrightarrow{K D}$. Marque o ponto $N$, interseção da reta $\overleftrightarrow{A M}$ com a reta $\overleftrightarrow{K D}$ e, em seguida, construa as retas $\overleftrightarrow{A D}$ e $\overleftrightarrow{L N}$. O ponto de interseção das retas $\overleftrightarrow{A D}$ e $\overleftrightarrow{L N}$ é o ponto $R$. 
Em seguida, construa o ponto $P$, dado pela interseção das retas $\overleftrightarrow{B S}$ e $\overleftrightarrow{R C}$, e o ponto $Q$, dado pela interseção das retas $\overleftrightarrow{A P}$ e $\overleftrightarrow{S C}$.

\section{Problema 2}

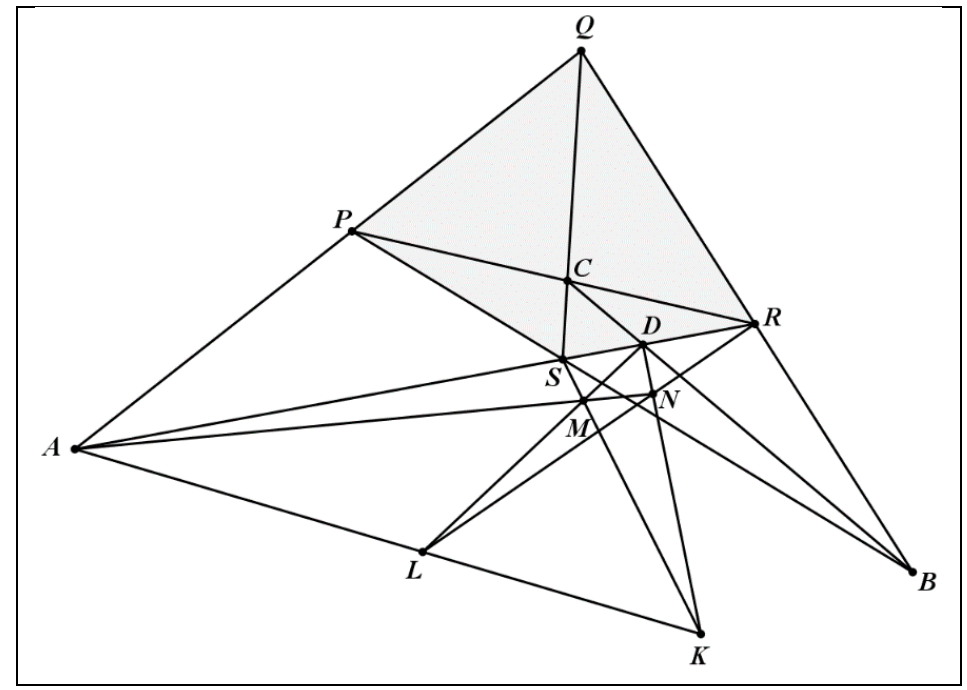

3. Suponha que a circunferência inscrita no triângulo $A B C$ tangencia os lados $B C, A C$ e $A B$ em $D, E$ e $F$, respectivamente. Seja $M$ um ponto do plano tal que a circunferência inscrita no triângulo $B C M$ tangencia $B C$ em $D$. Sejam $P$ e $Q$, respectivamente, os pontos onde a circunferência inscrita em $B C M$ tangencia os lados $B M$ e $C M$. Prove que as retas $\overleftrightarrow{E F}, \overleftrightarrow{P Q}$ e $\overleftrightarrow{B C}$ são concorrentes.

\section{Solução.}

As semirretas $\overrightarrow{A D}, \overrightarrow{B E}$ e $\overrightarrow{C F}$ são as bissetrizes internas do triângulo $A B C$. Usando o teorema de Ceva, conclui-se que as semirretas $\overrightarrow{A D}, \overrightarrow{B E}$ e $\overrightarrow{C F}$ são concorrentes em um ponto $G$, chamado de ponto de Gergonne do triângulo $A B C$. Seja $X$ o ponto de interseção das retas $\overleftrightarrow{B C}$ e $\overleftrightarrow{E F}$. Tem-se que $A F G E$ é um 
quadrilátero tal que $B=\stackrel{\leftarrow}{A} \vec{F} \cap \stackrel{\leftarrow}{G} \vec{E}, C=\stackrel{\leftarrow}{A} \vec{E} \cap \stackrel{\leftarrow}{F} \vec{G}, D=\stackrel{\leftrightarrow}{B} \vec{C} \cap$

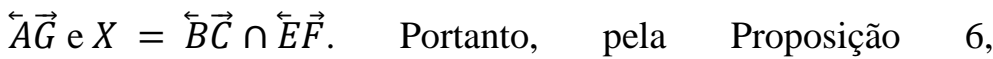
$H(B, C, D, X)$.

De modo análogo, tem-se $H(B, C ; D, Y)$, onde $Y=\overleftarrow{B} \vec{C} \cap \stackrel{t}{P} \vec{Q}$. Logo, $H(B, C ; D, X)$ e $H(B, C ; D, Y), \quad$ o que implica, $\mathrm{R}(\mathrm{B}, \mathrm{C} ; \mathrm{D}, \mathrm{X})=\mathrm{R}(\mathrm{B}, \mathrm{C} ; \mathrm{D}, \mathrm{Y})$, pelo Teorema 1 , temos que $X=Y$, o que demonstra que as retas $\stackrel{\leftrightarrow}{B C}, \stackrel{\leftrightarrow}{E} \vec{F}$ e $\stackrel{\leftrightarrow}{P}$ são concorrentes.

\section{Problema 3}

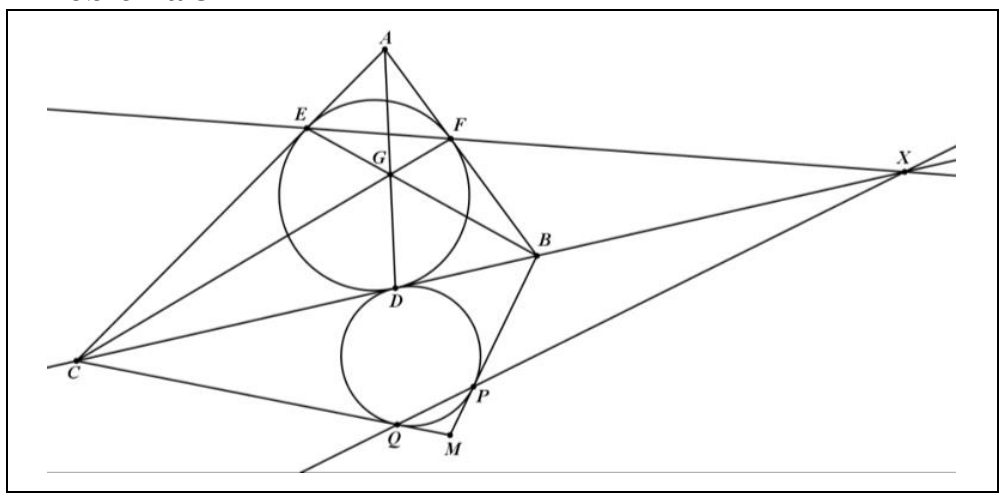

4. Dado um triângulo $A B C$, sejam $D$ e $E$ pontos no segmento $B C$ tais que $\overline{B D}=\overline{D E}=\overline{E C}$, sendo o ponto $D$ diferente dos pontos $B$ e $C$. A reta $p$ intersecta os segmentos $A B, A D, A E$ e $A C$ nos pontos $K, L, M$ e $N$, respectivamente. Prove que $\overline{K N} \geq 3 \overline{L M}$ e verifique que a igualdade ocorre se, e somente se, $\overline{K L}=\overline{L M}=\overline{M N}$.

\section{Solução.}

Sejam $x=\overline{K L}, y=\overline{L M} \mathrm{e} z=\overline{M N}$. Temos que provar que $x+y+z \geq 3 y$, ou equivalentemente, $x+z \geq 2 y$. As quatro retas $\overleftrightarrow{A B}, \overleftrightarrow{A D}, \stackrel{\leftrightarrow}{A} \vec{E}$ e $\overleftrightarrow{A C}$ são concorrente em $A$ e, pelo 
enunciado do problema, $K=\overleftrightarrow{A B} \cap p, L=\overleftrightarrow{A D} \cap p, \quad M=$ $\overleftrightarrow{A E} \cap p$ e $N=\overleftrightarrow{A C} \cap p$, como mostra a figura abaixo.

\section{Problema 4}

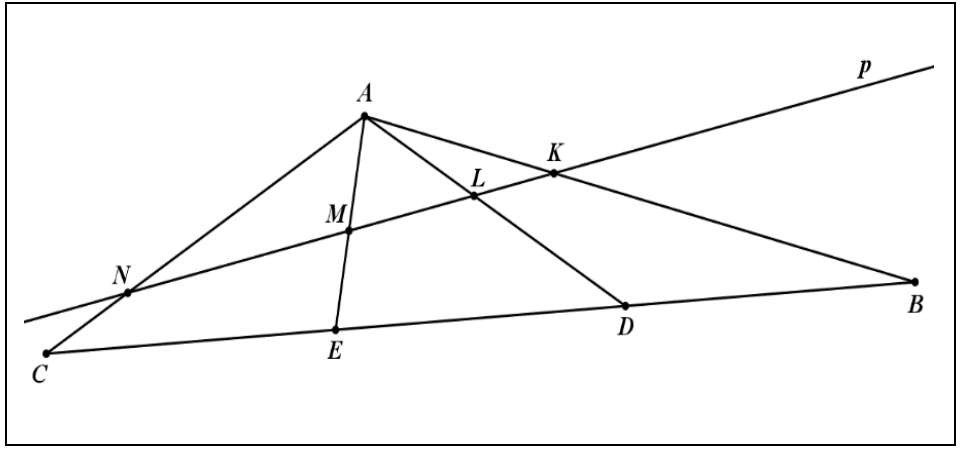

Logo, $K L M N \stackrel{\stackrel{A}{=}}{\wedge} B D E C$. Como a perspectividade preserva a razão cruzada, por isso, $R(K, L ; M, N)=R(B, D ; E, C)$, pelas Propriedades 5 e 4 , respectivamente, temos

$$
R(K, N ; L, M)=R(B, C ; D, E) .
$$

Logo,

$$
\frac{\overline{K L}}{\overline{L N}} \overline{\overline{K M}}=\frac{\overline{B D}}{\overline{\overline{D C}}} \overline{\overline{B C}}
$$

e portanto,

$$
\frac{X}{(Y+Z)} \frac{Z}{(X+Y)}=\frac{1}{2} \frac{1}{2}
$$

o que implica

$$
4 x z=(x+y)(y+z) .
$$

Suponha que $y>\frac{x+z}{2}$.

Tem-se

$$
x+y>\frac{3 x+z}{2}=\frac{2(x+x+x+z)}{4} \geq 2 \sqrt[4]{x x x z} .
$$


A última desigualdade ocorre porque a média aritmética de números reais positivos é maior ou igual a média geométrica desses números. Analogamente,

$$
z+y>2 \sqrt[4]{x z z z}
$$

Assim,

$$
(x+y)(y+z)>2 \sqrt[4]{x x x z} \cdot 2 \sqrt[4]{x z z z}=4 x y,
$$

o que contradiz (1).

Portanto, $y \leq \frac{x+z}{2}$, ou seja, $\mathrm{x}+\mathrm{z} \geq 2 \mathrm{y}$.

Analisemos, agora, o caso da igualdade.

Se $y=\frac{x+z}{2}$, então

$$
\begin{aligned}
4 x z & =(x+y)(y+z) \\
& =\frac{(3 x+z)(x+3 z)}{4},
\end{aligned}
$$

e portanto

$$
16 x z=(3 x+z)(x+3 z),
$$

o que implica

$$
3(x-z)^{2}=0,
$$

ou seja,

$$
x=z .
$$

Substituindo $x=z$ em $y=\frac{x+z}{2}$, temos $y=z$.

Portanto, a igualdade é verdadeira se, $\overline{K L}=\overline{L M}=\overline{M N}$.

Se $\overline{K L}=\overline{L M}=\overline{M N}$, então $\overline{K M}=\overline{K L}+\overline{L M}+\overline{M N}$.

Assim, temos $\overline{K M}=3 \overline{L M}$ se, e somente se, $\overline{K L}=\overline{L M}=\overline{M N}$.

5. O ponto $M_{1}$ pertence ao lado $A B$ do quadrilátero $A B C D$. Os pontos $M_{2}, M_{3}, \ldots$ são definidos da seguinte forma: $M_{2}$ é a interseção da reta $\overleftrightarrow{B C}$ com a reta $\overleftrightarrow{D M_{1}}, M_{3}$ é a interseção da reta $\overleftrightarrow{C D}$ com a reta $\overleftrightarrow{A M_{2}}, M_{4}$ é a interseção da reta $\overleftrightarrow{D A}$ com a reta $\overleftrightarrow{B M_{3}}$, e assim por diante. Prove que $M_{13}=M_{1}$. 


\section{Solução.}

Sejam $E=\overleftrightarrow{A B} \cap \overleftrightarrow{C D}$ e $F=\overleftrightarrow{A D} \cap \overleftrightarrow{B C}$. Considere a sequência.

$$
A B E M_{1} \stackrel{\underline{D}}{\wedge} F B C M_{2} \stackrel{\underline{A}}{\wedge} D E C M_{3} \stackrel{\underline{\underline{B}}}{\wedge} D_{A F M_{4}} \stackrel{\underline{C}}{\wedge} E A B M_{5} .
$$

De acordo com as condições dadas no problema, esta sequência de perspectividades tem que ser aplicada mais duas vezes para se chegar ao ponto $M_{13}$. Observe que a sequência de perspectividades é a projetividade $A B E M_{1} \bar{\wedge} E A B M_{5}$, que leva $A$ em $E, B$ em $A$ e $E$ em $B$. Claramente, se aplicamos a sequência de perspectividades três vezes, os pontos $A, B$ e $C$ serão deixados fixos, enquanto $M_{1}$ será projetado em $M_{13}$. De fato:

Na primeira sequência, obtemos

$$
A B E M_{1} \bar{\wedge} E A B M_{5} .
$$

Na segunda, obteremos

$$
E A B M_{5} \bar{\wedge} B E A M_{9}
$$

E na terceira, obteremos

$$
B E A M_{9} \bar{\wedge} A B E M_{13} .
$$

Por (1), (2) e (3), concluímos que $A B E M_{1} \bar{\wedge} A B E M_{13}$.

Pelo Corolário 1, essa projetividade é a identidade.

Portanto, $M_{13}=M_{1}$. 


\section{Problema 5}

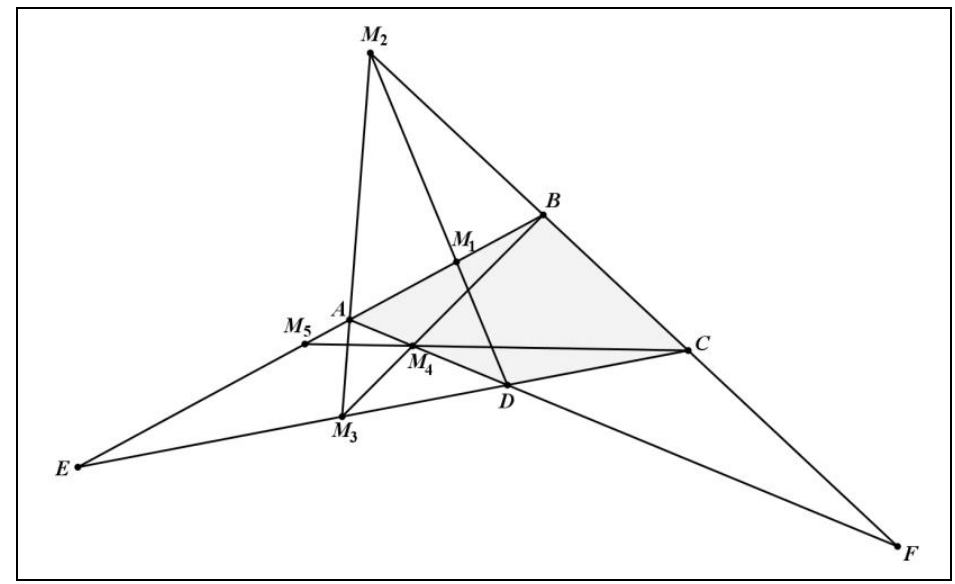

6. (Teorema da Borboleta) Os pontos $M$ e $N$ pertencem à circunferência $C(O, r)$. Seja $P$ o ponto médio da corda $M N$ e sejam $A B$ e $C D$ ( $A$ e $C$ em um mesmo lado de $M N$ ) cordas arbitrárias da circunferência $C(O, r)$ passando por $P$. Prove que as cordas $A D$ e $B C$ intersectam $M N$ em pontos equidistantes de $P$.

\section{Solução.}

Considere os pontos $Y=M N \cap B C$ e $X=M N \cap A D$, como mostra a figura abaixo. 


\section{Problema 6}

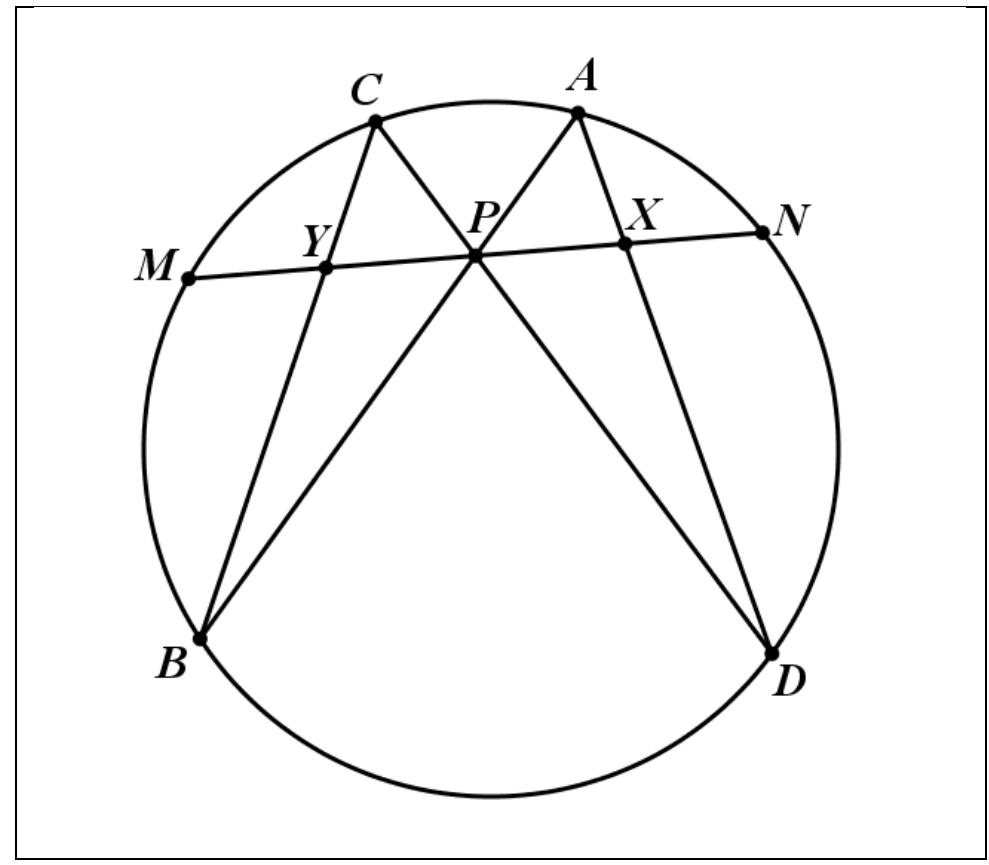

Seja $Z$ o ponto simétrico de $Y$ em relação ao ponto $P$. Temos que provar que $X=Z$.

Considere a sequência de perspectividades

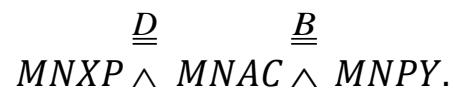

Como a razão cruzada é preservada por perspectividade, temos $R(M, N ; X, P)=R(M, N ; P, Y)$. Pela Definição 1 , por $\overline{M P}=$ $\overline{N P}, \overline{Y N}=\overline{Z M}, \overline{M Y}=\overline{N Z}$ e pelos pares de segmentos com o mesmo comprimento terem sentidos opostos, temos

$$
\begin{aligned}
R(M, N ; P, Y) & =\frac{\overline{M P}}{\overline{P N}} \overline{\overline{Y N}} \\
& =1 \cdot \frac{\overline{Z M}}{\overline{N Z}}
\end{aligned}
$$




$$
\begin{aligned}
& =\frac{\overline{N P}}{\overline{P M}} \frac{\overline{Z M}}{\overline{N Z}} \\
& =R(N, M ; P, Z) .
\end{aligned}
$$

Pela Propriedade 3, temos

$$
R(N, M ; P, Z)=R(M, N ; Z, P) .
$$

Portanto, $R(M, N ; X, P)=R(M, N ; Z, P)$. Isto implica, pela Propriedade 5, $R(M, N ; P, X)=R(M, N ; P, Z)$.

Pelo Teorema 1 , temos $X=Z$.

7. Dado um triângulo $A B C$, sejam $D$ e $E$ pontos dos lados $A B$ e $A C$, respectivamente, tais que $\overleftrightarrow{D E} \| \overleftrightarrow{B C}$. Considere $P$ um ponto no interior do triângulo $A D E$. Suponha que as retas $\overleftrightarrow{B P} \mathrm{e}$ $\overleftrightarrow{C P}$ intersectam a reta $\overleftrightarrow{D E}$ em $F$ e $G$, respectivamente. As circunferências circunscritas aos triângulos $P D G$ e $P F E$ se intersectam em $P$ e $Q$. Prove que os pontos $A, P$ e $Q$ são colineares.

\section{Solução.}

Sejam $J=D Q \cap B P, \quad K=E Q \cap C P \quad$ e $\quad T=D E \cap P Q$. Primeiramente, vamos provar que $\overleftrightarrow{J K} \| \overleftrightarrow{D E}$.

Aplicando o teorema de Menelaus nos triângulos DTQ e ETQ em relação às retas $\overleftrightarrow{P F}$ e $\overleftrightarrow{P G}$, respectivamente, obtemos

$$
\frac{\overline{D J}}{\overline{\overline{J Q}}} \frac{\overline{Q P}}{\overline{P T}} \frac{\overline{T F}}{\overline{F D}}=1
$$

e

$$
\frac{\overline{E K}}{\overline{\bar{K} Q}} \frac{\overline{Q P}}{\overline{P T}} \frac{\overline{T G}}{\overline{G E}}=1
$$

Dividindo (1) por (2), tem-se 


$$
\overline{\overline{D J}} \frac{\overline{Q P}}{\overline{P Q}} \frac{\overline{T F}}{\overline{P Q} \overline{F D}} \frac{\overline{P T}}{\overline{E K}} \frac{\overline{G E}}{\overline{Q P}} \frac{\overline{D J}}{\overline{T G}}=\frac{\overline{T F}}{\overline{J Q}} \overline{\overline{K Q}} \frac{\overline{G E}}{\overline{F K}} \frac{\overline{T S}}{\overline{T G}}=1 .
$$

O ponto $P$ está no eixo radical das circunferências circunscritas aos triângulos $D T Q$ e $E T Q$, por isso,

$$
\frac{\overline{D T}}{\overline{T E}}=\frac{\overline{F T}}{\overline{T G}} \Rightarrow \frac{\overline{F T}}{\overline{T G}}=\frac{\overline{D T}-\overline{F T}}{\overline{T E}-\overline{T G}}=\frac{\overline{F D}}{\overline{G E}} \Rightarrow \frac{\overline{F T}}{\overline{F D}} \frac{\overline{G E}}{\overline{T G}}=1 .
$$

Substituindo (4) em (3), temos

$$
\frac{\overline{D J}}{\overline{J Q}} \frac{\overline{T F}}{\overline{F D}} \frac{\overline{K Q}}{\overline{E K}} \frac{\overline{G E}}{\overline{T G}}=\frac{\overline{D J}}{\overline{J Q}} \frac{\overline{K Q}}{\overline{E K}}=1 \Rightarrow \frac{\overline{D J}}{\overline{J Q}}=\frac{\overline{E K}}{\overline{K Q}} .
$$

Logo, a reta $\overleftrightarrow{J K}$ divide os lados do triângulo $Q D E$ em segmentos proporcionais, mostrando que os triângulos $Q D E$ e $Q J K$ são semelhantes. Portanto, as retas $\overleftrightarrow{J K}$ e $\overleftrightarrow{D E}$ são paralelas, como mostra a figura abaixo. 


\section{Problema 7}

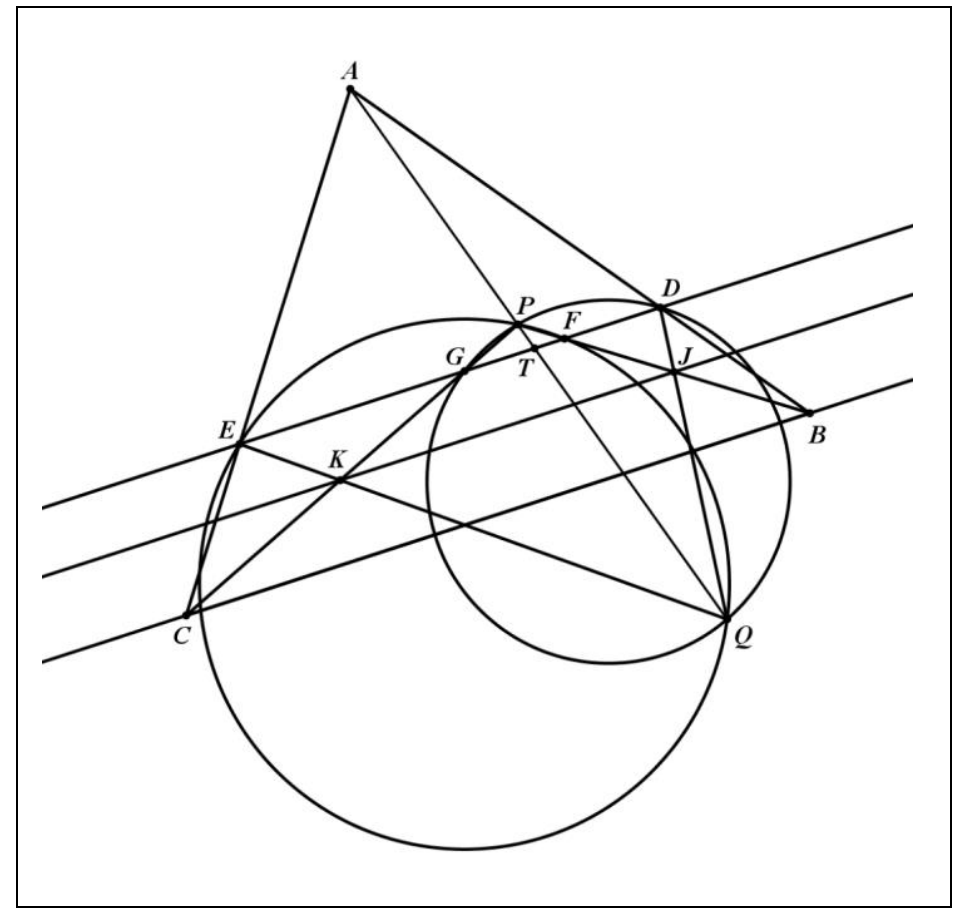

Como as retas $\overleftrightarrow{D E}, \overleftrightarrow{J K}$ e $\overleftrightarrow{B C}$ são paralelas e contêm os vértices correspondentes dos triângulos $D J B$ e $E K C$, temos que esses triângulos estão em perspectivas em relação a um ponto no infinito. Portanto, pelo Teorema de Desargues, os pontos $Q=\overleftrightarrow{D J} \cap \overleftrightarrow{E K}, P=\overleftrightarrow{B J} \cap \overleftrightarrow{C K}$ e $A=\overleftrightarrow{B D} \cap \overleftrightarrow{C E}$ são colineares.

8. Seja $A_{1} A_{2} A_{3}$ um triângulo não isósceles de incentro $I$. Seja $C_{i}, i=1,2,3$, a menor circunferência através de $I$ tangente a ambos $A_{i} A_{i+1}$ e $A_{i} A_{i+2}$ (os índices são tomados módulo 3 ). Seja $B_{i}, i=1,2,3$, o outro ponto de interseção de $C_{i+1}$ e $C_{i+2}$. Demonstre que os circuncentros dos triângulos $A_{1} B_{1} I, A_{2} B_{2} I$ e $A_{3} B_{3} I$ são colineares. 


\section{Solução.}

Para $i=1,2,3$, denotamos por $O_{i}$ o centro da circunferência $C_{i}$ e por $M_{i}$ o ponto médio do arco $A_{l+1} A_{l+2}$ que não contém o ponto $A_{i}$. Primeiramente, temos que a reta $\overleftrightarrow{I B_{l}}$ é o eixo radical das circunferências $C_{i+1}$ e $C_{i+2}$. Como o eixo radical de duas circunferências é perpendicular à reta que contém os centros das duas circunferências, temos $\overleftrightarrow{I B_{l}} \perp \overleftrightarrow{O_{l+1} O_{l+2}}$. Como o segmento $I B_{i}$ é uma corda das circunferência $C_{i+1}$ e $C_{i+2}$, então a reta $\overleftrightarrow{O_{l+1} O_{l+2}}$ é a mediatriz do segmento $I B_{i}$, e portanto, contém o circuncentro $R_{i}$ do triângulo $A_{i} B_{i} I$.

Sendo $\overleftrightarrow{A_{l} I}$ a bissetriz interna do triângulo $A_{1} A_{2} A_{3}$ e $M_{i}$ o ponto médio do arco $A_{l+1} A_{l+2}$ que não contém o ponto $A_{i}$, temos que $M_{i}$ pertence a bissetriz $\overleftrightarrow{A_{l} I}$, o que implica, $\overleftrightarrow{M_{l} A_{l}}=\overleftrightarrow{M_{l} I}$. Com isso, a reta $\overleftrightarrow{M_{l} M_{l+1}}$ é a mediatriz do segmento $A_{i+2} I$. Logo, $R_{i+2}$ pertence à reta $\overleftrightarrow{M_{l} M_{l+1}}$.

Portanto, $R_{i}=\overleftrightarrow{O_{l+1} O_{l+2}} \cap \overleftrightarrow{M_{l} M_{l+1}}$. As retas $\overleftrightarrow{O_{1} M_{1}}, \overleftrightarrow{O_{2} M_{2}}$ e $\overleftrightarrow{O_{3} M_{3}}$ são concorrentes em $I$. Daí, os triângulos $O_{1} O_{2} O_{3}$ e $M_{1} M_{2} M_{3}$ estão em perspectiva em relação ao ponto $I$. Pelo Teorema de Desargues, os pontos de interseção das retas $\overleftrightarrow{O_{l+1} O_{l+2}}$ e $\overleftrightarrow{M_{l} M_{l+1}}$, ou seja, os $R_{i}$, são colineares. 


\section{Problema 8}

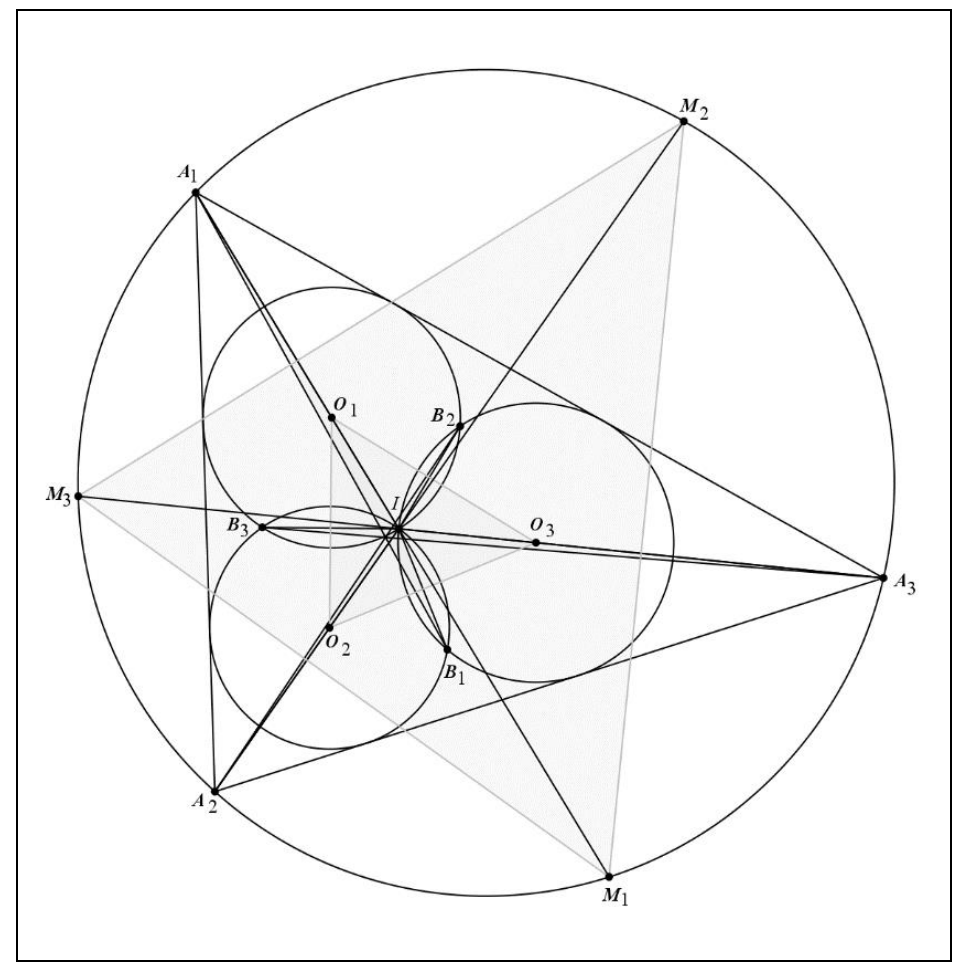

9. Sejam $C_{a}, C_{b}$ e $C_{c}$ circunferências no interior do triângulo $A B C$, que são tangentes (externamente) uma à outra, com, $C_{a}$ tangente a $A B$ e $A C, C_{b}$ tangente a $B A$ e $B C$ e $C_{c}$ tangente a $C A$ e $C B$. Seja $D$ o ponto comum de $C_{b}$ e $C_{c}, E$ o ponto comum de $C_{c}$ e $C_{a}$, e $F$ o ponto comum de $C_{a}$ e $C_{b}$. Mostre que $\overleftrightarrow{A D}, \overleftrightarrow{B E}$, $\overleftrightarrow{C F}$ são concorrentes.

\section{Solução.}

Sejam $A_{1}, B_{1}$ e $C_{1}$ os centros dos círculos $C_{a}, C_{b}$ e $C_{c}$, respectivamente. Sejam $R=\overleftrightarrow{E F} \cap \overleftrightarrow{B_{1} C_{1}}, S=\overleftrightarrow{E D} \cap \overleftrightarrow{A_{1} B_{1}}$ e $T=\overleftrightarrow{F D} \cap \overleftrightarrow{A_{1} C_{1}}$. Com isso, $R$ é o centro externo de 
similaridade dos círculos $C_{b}$ e $C_{c}, S$ é o centro externo de similaridade dos círculos $C_{a}$ e $C_{b}$ e $T$ é o centro externo de similaridade dos círculos $C_{a}$, e $C_{c}$. Então, pelo Teorema de Monge, os pontos $R, S$ e $T$ são colineares. Portanto, os triângulos $A B C$ e $D E F$ estão em perspectiva em relação à reta $\overleftrightarrow{R S}$. Pelo Teorema de Desargues, esses triângulos estão em perspectivas em relação a um ponto, ou seja, as retas $\overleftrightarrow{A D}, \overleftrightarrow{B E}$, $\overleftrightarrow{C F}$ são concorrentes.

\section{Problema 9}

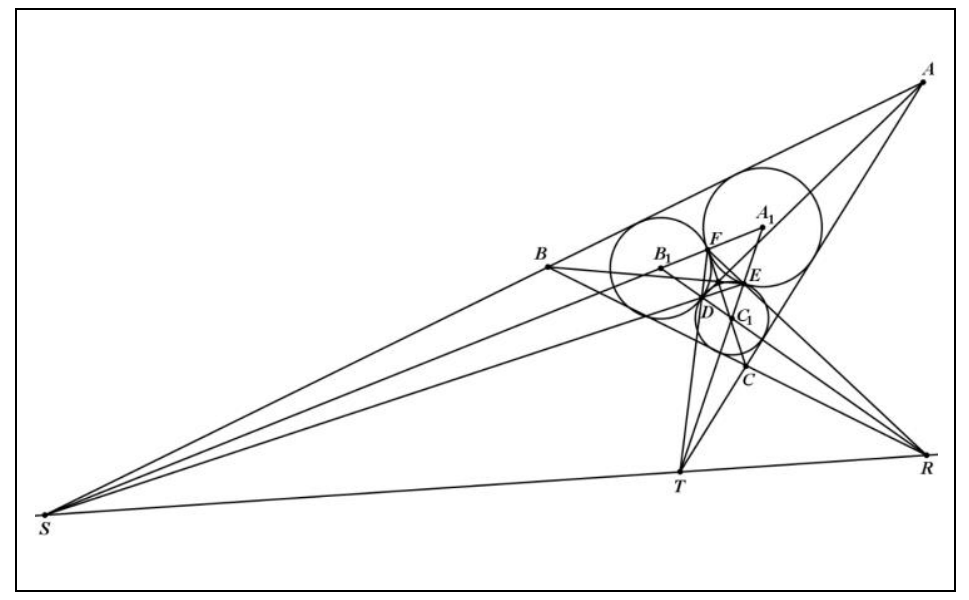

10. Seja $\Gamma$ a circunferência circunscrita ao triângulo $A B C$. A circunferência $\Gamma_{1}$ é tangente a $B C, C A$ e $A B$ em $D, E, F$, respectivamente. Uma circunferência $\Gamma_{A}$ é tangente a $B C$ em $D$ e a $\Gamma$ em $A_{1}$, de modo que $A_{1}$ e $A$ estão em lados diferentes de $B C$. Define-se $B_{1}$ e $C_{1}$ similarmente. Prove que as retas $\overleftrightarrow{D A_{1}}$, $\overleftrightarrow{E B_{1}}$ e $\overleftrightarrow{F C_{1}}$ são concorrentes.

\section{Solução.}

Seja $T$ o centro interno de similaridade das circunferência $\Gamma$ e $\Gamma_{1}$. Aplicando o Teorema de Monge e D'Alembert nas 
circunferência $\Gamma, \Gamma_{1}$ e $\Gamma_{A}$, obtemos que os pontos $T, A_{1}$ e $D$ são colineares.

O ponto $E$ é o centro interno de similaridade das circunferência $\Gamma_{1}$ e $\Gamma_{B}$ e $B_{1}$ é o centro externo de similaridade das circunferência $\Gamma$ e $\Gamma_{B}$, pelo teorema de Monge e D'Alembert, os pontos $E, B_{1}$ e $T$ são colineares.

Analogamente, temos que os pontos $F, T$ e $C_{1}$ são colineares.

Portanto, as retas $\overleftrightarrow{D A_{1}}, \overleftrightarrow{E B_{1}}$ e $\overleftrightarrow{F C_{1}}$ são concorrentes no ponto $T$.

\section{Problema 10}

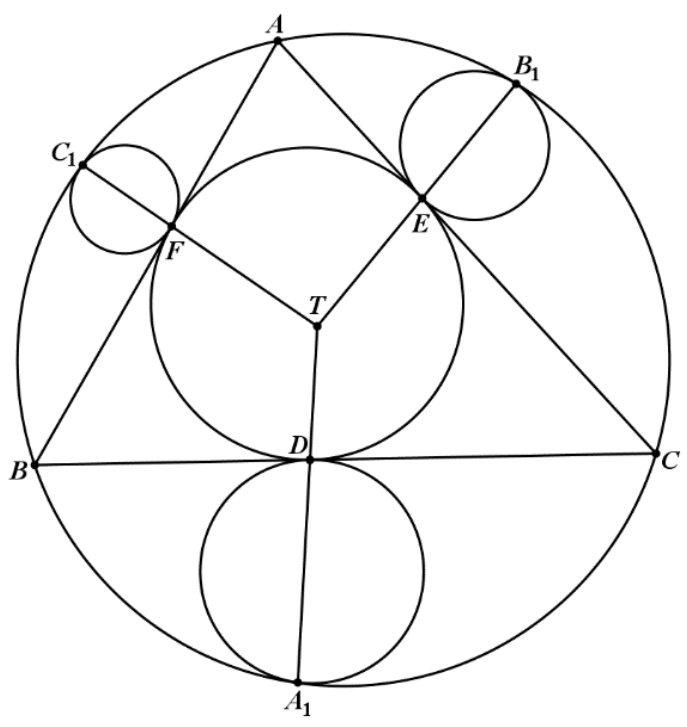

11. O ponto $P$ encontra-se no lado $A B$ de um quadrilátero convexo $A B C D$. Seja $\Gamma_{C}$ o círculo inscrito ao triângulo $C P D$, e $I$ seu incentro. Suponha que $\Gamma_{C}$ é tangente aos círculos inscritos aos triângulos $A P D$ e $B P C$ nos pontos $K$ e $L$, respectivamente. As diagonais $A C$ e $B D$ se encontram em $E$, e as retas $A K$ e $B L$ 
se encontram em $F$. Prove que os pontos $E, I$ e $F$ são colineares.

\section{Solução.}

Seja $\Gamma$ o círculo tangente aos segmentos $A B, B C$ e $A D$ e sejam $\Gamma_{A}$ e $\Gamma_{B}$ os círculos inscritos aos triângulos $A P D$ e $B P C$, respectivamente. Dados que $A$ é o centro externo de similaridade dos círculos $\Gamma_{A}$ e $\Gamma$, e $K$ é o centro interno de similaridade dos círculos $\Gamma_{A}$ e $\Gamma_{C}$, pelo teorema de Monge e D'Alembert, a reta $\overleftrightarrow{A K}$ intersecta o segmento $O I$ no centro interno de similaridade dos círculos $\Gamma$ e $\Gamma_{C}$, onde $O$ é o centro do círculo $\Gamma$. Da mesma forma, a reta $\overleftrightarrow{B L}$ intersecta o segmento $O I$ no centro interno de similaridade $F$ dos círculos $\Gamma$ e $\Gamma_{C}$. Agora basta provar que o ponto $E$ pertence à reta $\overleftrightarrow{O I}$. Sejam $M=A P \cap \Gamma_{A}, R=A D \cap \Gamma_{A}$ e $N=C D \cap \Gamma_{C}$. Pelas propriedades das retas tangentes, temos

$$
\begin{aligned}
A P+C D & =A M+M P+C N+N D \\
& =A R+P K+C L+D K \\
& =A R+D R+P L+C L \\
& =P C+A D .
\end{aligned}
$$

De maneira análoga,

$$
B P+C D=P D+B C .
$$

Por isso, existem círculos $\Gamma_{1}$ e $\Gamma_{2}$ inscritos nos quadriláteros $A P C D$ e $B C P D$, respectivamente.

Seja $X$ o centro externo de similaridade dos círculos $\Gamma_{A}$ e $\Gamma_{C}$ e $Y$ o centro externo de similaridade dos círculos $\Gamma_{B}$ e $\Gamma_{C}$. Pelo Teorema de Monge aplicado aos círculos $\Gamma_{A}, \Gamma_{C}$ e $\Gamma_{1}$, segue que os pontos $A, C$ e $X$ são colineares. Invocando o Teorema de Monge com os círculos $\Gamma_{B}, \Gamma_{C}$ e $\Gamma_{2}$, temos que os pontos $B, D$ e $Y$ são colineares.

Seja $E_{1}$ o centro externo de similaridade dos círculos $\Gamma$ e $\Gamma_{C}$. Pelo Teorema Monge aplicado aos círculos $\Gamma$ e $\Gamma_{A}$ e $\Gamma_{C}$, segue 
que os pontos $A, X$ e $E_{1}$ são colineares. Então, $E_{1} \in A C$ e $E_{1} \in \overleftrightarrow{O I}$. Analogamente, $E_{1} \in B D$ e $E_{1} \in \overleftrightarrow{O I}$. Portanto, $E_{1}=E$ e $E, I$ e $F$ são colineares.

\section{Problema 11}

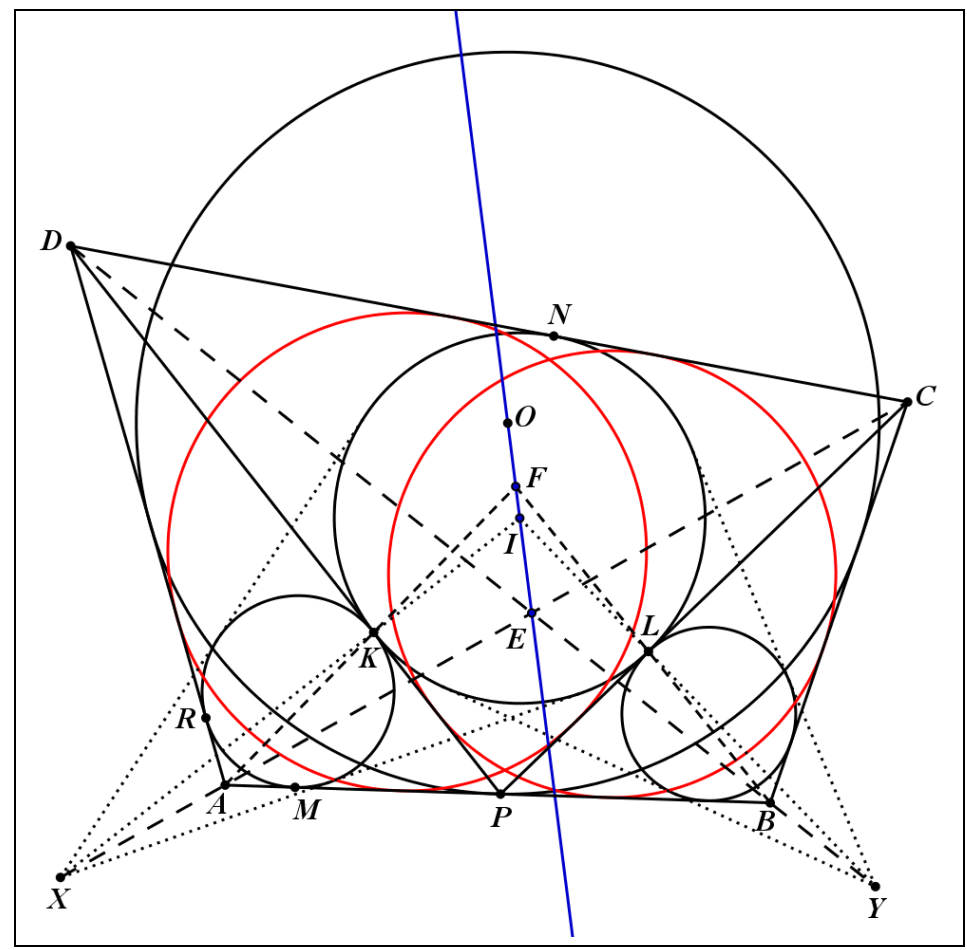

12. Dado um triângulo $A B C$ e um ponto $T$, sejam $P$ e $Q$ os pés das perpendiculares de $T$ em relação às retas $\overleftrightarrow{A B}$ e $\overleftrightarrow{A C}$, respectivamente. Sejam $R$ e $S$ os pés das perpendiculares de $A$ em relação às retas $\overleftrightarrow{T C}$ e $\overleftrightarrow{T B}$, respectivamente. Prove que a interseção das retas $\overleftrightarrow{P R}$ e $\overleftrightarrow{Q S}$ pertence à reta $\overleftrightarrow{B C}$.

Solução. 
Temos que provar que o ponto $X=\overleftrightarrow{P R} \cap \overleftrightarrow{Q S}$ pertence à reta $\overleftrightarrow{B C}$.

Pelo enunciado do problema, temos

$$
\angle A P T=\angle A S T=\angle A Q T=\angle A R T=90^{\circ} .
$$

Logo, a circunferência que possui diâmetro $A T$, contém os pontos $P, S, Q$ e $R$.

Portanto, aplicando o Teorema de Pascal, concluímos que os pontos $\quad B=\overleftrightarrow{A P} \cap \overleftrightarrow{S T}, C=\overleftrightarrow{A Q} \cap \overleftrightarrow{R T}$ e $\quad X=\overleftrightarrow{P R} \cap \overleftrightarrow{Q S}$ são colineares.

\section{Problema 12}

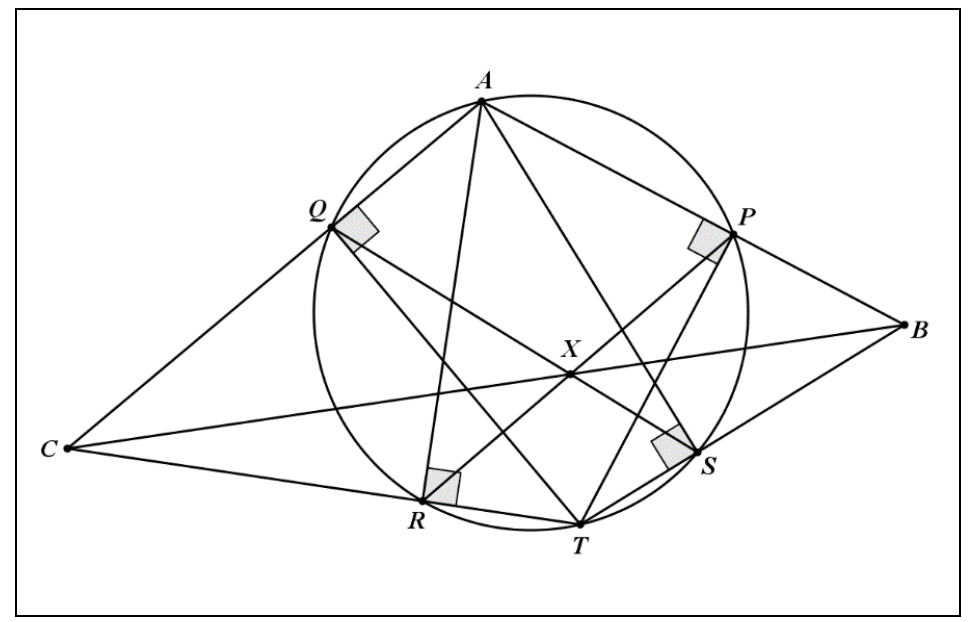

13. São dados um triângulo $A B C$ e um ponto $M$. Uma reta que passa por $M$ intersecta as retas $\overleftrightarrow{A B}, \overleftrightarrow{B C}$, e $\overleftrightarrow{A C}$ em $C_{1}, A_{1}$ e $B_{1}$, respectivamente. As retas $\overleftrightarrow{A M}, \overleftrightarrow{B M}$ e $\overleftrightarrow{C M}$ intersectam a circunferência circunscrita ao triângulo $A B C$, respectivamente, em $A_{2}, B_{2}$ e $C_{2}$. Prove que as retas $\overleftrightarrow{A_{1} A_{2}}, \overleftrightarrow{B_{1} B_{2}}$ e $\overleftrightarrow{C_{1} C_{2}}$ se intersectam em um ponto que pertence à circunferência circunscrita ao triângulo $A B C$.

Solução. 
Suponha que a reta $\overleftrightarrow{A_{1} A_{2}}$ intersecta a circunferência circunscrita ao triângulo $A B C$ em $A_{2}$ e $X$. Seja $Y=\overleftrightarrow{X B_{2}} \cap \overleftrightarrow{A C}$. Com isso, os pontos $A, B, C, A_{2}, B_{2}$ e $X$ pertencem à circunferência circunscrita ao triângulo $A B C$. Aplicando o Teorema de Pascal a esses pontos, obtemos que os pontos $M=\overleftrightarrow{A A_{2}} \cap \overleftrightarrow{B B_{2}}$, $Y=\overleftrightarrow{A C} \cap \overleftrightarrow{X B_{2}}$ e $A_{1}=\overleftrightarrow{B C} \cap \overleftrightarrow{X A_{2}}$ são colineares. Daí, o ponto $Y$ pertence à reta $\overleftrightarrow{A_{1} M}$. De acordo com a definição do ponto $Y$, este pertencente à reta $\overleftrightarrow{A C}$. Portanto, $Y=\overleftrightarrow{A_{1} M} \cap \overleftrightarrow{A C}=B_{1}$. Logo, $B_{1}=\overleftrightarrow{X B_{2}} \cap \overleftrightarrow{A C}$, o que mostra que os pontos $X$, $B_{1}$ e $B_{2}$ são colineares.

Analogamente, mostramos que os pontos $X, C_{1}$ e $C_{2}$ são colineares. Portanto, as retas $\overleftrightarrow{A_{1} A_{2}}, \overleftrightarrow{B_{1} B_{2}}$ e $\overleftrightarrow{C_{1} C_{2}}$ se intersectam no ponto $X$, o qual pertence ao círculo circunscrito ao triângulo $A B C$.

\section{Problema 13}

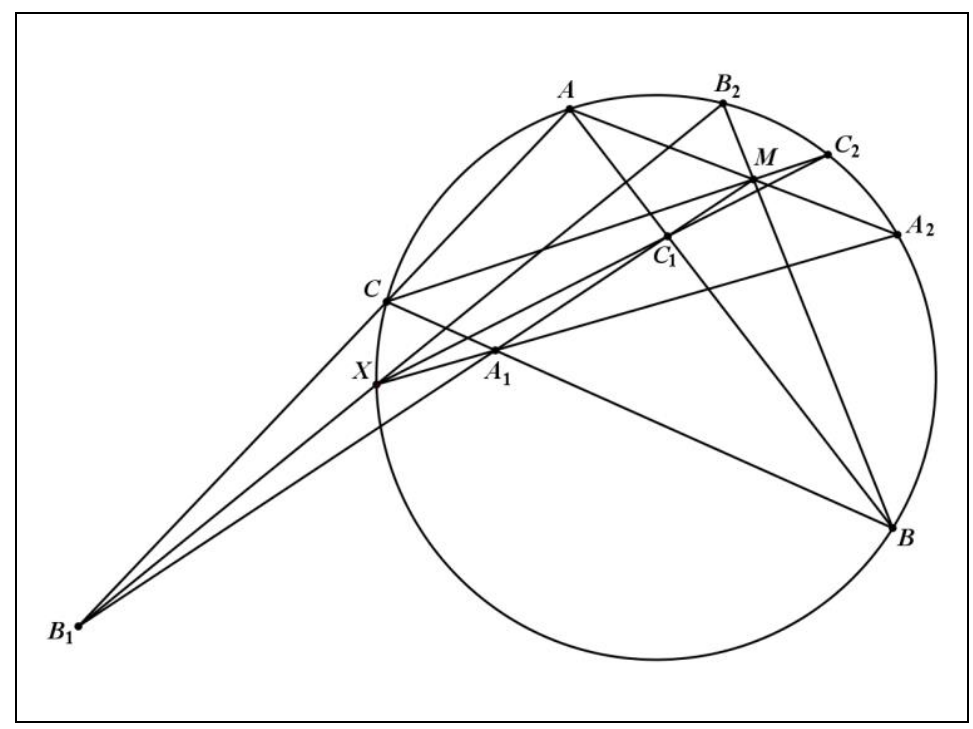


14. Sejam $P$ e $Q$ pontos conjugados isogonais e suponha que os triângulos $P_{1} P_{2} P_{3}$ e $Q_{1} Q_{2} Q_{3}$ são os seus triângulos pedais. Sejam $X_{1}=\overleftrightarrow{P_{2} Q_{3}} \cap \overleftrightarrow{P_{3} Q_{2}}, X_{2}=\overleftrightarrow{P_{1} Q_{3}} \cap \overleftrightarrow{P_{3} Q_{1}}$ e $X_{3}=\overleftrightarrow{P_{1} Q_{2}}$ $\cap \overleftrightarrow{P_{2} Q_{1}}$. Prove que os pontos $X_{1}, X_{2}$ e $X_{3}$ pertencem à reta $\overleftrightarrow{P Q}$.

\section{Solução.}

Pelas propriedades dos triângulos pedais, temos que os triângulos pedais em relação aos pontos conjugados isogonais $P$ e $Q$ possuem o mesmo circuncírculo, chamado de círculo pedal, e o centro desse círculo é o ponto médio dos pontos $P$ e $Q$. Denotamos por $R$ esse ponto médio. Sejam $P_{4}=\overleftrightarrow{P P_{1}} \cap \overleftrightarrow{Q_{1} R} \mathrm{e}$ $P_{5}=\overleftrightarrow{P P_{2}} \cap \overleftrightarrow{Q_{2} R}$. Os pontos $P_{4}$ e $P_{5}$ pertencem ao círculo pedal, pois as retas $\overleftrightarrow{Q_{1} R}$ e $\overleftrightarrow{Q_{2} R}$ passam pelo centro $R, \overleftrightarrow{P_{1} P} \perp \overleftrightarrow{Q_{1} P_{1}}$ e $\overleftrightarrow{P_{2} P} \perp \overleftrightarrow{Q_{2} P_{2}}$. Usando o teorema de Pascal nos pontos $P_{2}, P_{4}$, $Q_{2}, P_{1}, P_{5}$ e $Q_{1}$, obtemos que os pontos $P=\overleftrightarrow{P_{2} P_{5}} \cap \overleftrightarrow{P_{4} P_{1}}$, $X_{3}=\overleftrightarrow{P_{1} Q_{2}} \cap \overleftrightarrow{P_{2} Q_{1}}$ e $R=\overleftrightarrow{P_{4} Q_{1}} \cap \overleftrightarrow{Q_{2} P_{5}}$ são colineares, o que implica que $X_{3} \in \overleftrightarrow{P Q}$. Analogamente, os pontos $X_{1}$ e $X_{2}$ pertencem à reta $\overleftrightarrow{P Q}$. Portanto, os pontos $X_{1}, X_{2}$ e $X_{3}$ pertencem à reta $\overleftrightarrow{P Q}$. 


\section{Problema 14}

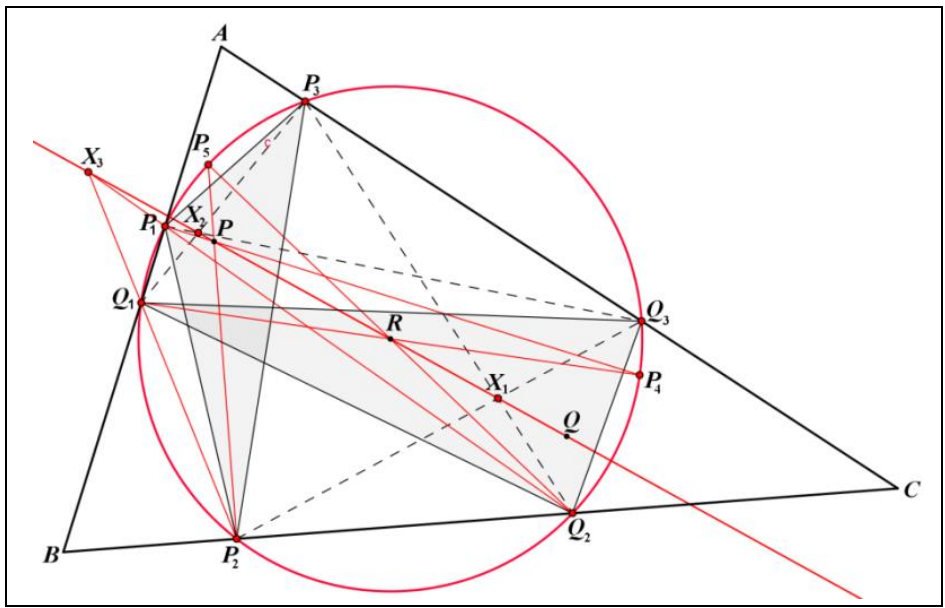

15. Se os pontos $A$ e $M$ são conjugados com respeito à circunferência $C$, então a circunferência com diâmentro $A M$ é ortogonal à circunferência $C$.

\section{Solução.}

Uma circunferência $\Gamma$ é invariante pela inversão em relação a uma circuferência $C$ se, e semomente se, $\Gamma=C$ ou $\Gamma \perp C$.

Primeiramente, vamos mostrar que a circunferência $C_{1}$, com diâmentro $A M$, é invariante pela inversão em relação à circunferência $C$.

Uma vez que o ponto $M$ pertence ao polar do ponto $A$ em relação à circunferência $C$, temos $\angle M A^{*} A=90^{\circ}$, onde $A^{*}$ é o inverso do ponto $A$ em relação à circunferência $C$.

Portanto, $A^{*} \in C_{1}$. Analogamente, $M^{*} \in C_{1}$, onde $M^{*}$ é o inverso do ponto $M$ em relação à circuferência $C$.

Seja $C_{1}{ }^{*}$ a imagem de $C_{1}$ pela inversão em relação à circunferência $C$. Como $A \in C_{1}$, temos que $A^{*} \in C_{1}{ }^{*}$. Como $A$ é o inverso de $A^{*}$ e $A^{*} \in C_{1}$, obtemos que $A \in C_{1}{ }^{*}$. 
Da mesma forma, obtemos $M \in C_{1}{ }^{*}$ e $M^{*} \in C_{1}{ }^{*}$.

Observe que as circunferências $C_{1}$ e $C_{1}{ }^{*}$ têm os quatros pontos $A, A^{*}, M$ e $M^{*}$ em comum.

Daí, $C_{1}=C_{1}{ }^{*}$ e, de acordo com a afirmação mencionada inicialmente, concluímos $C_{1}=C$ ou $C_{1} \perp C$.

$\mathrm{O}$ caso em que $C_{1}=C$ não pode ocorrer, pois a circunferência $C_{1}$ tem diâmentro $A M$ e este não pode ser o diâmentro de $C$, visto que $A$ e $M$ são conjugados.

Assim, $C_{1} \perp C$.

\section{Figura 35 - Problema 15}

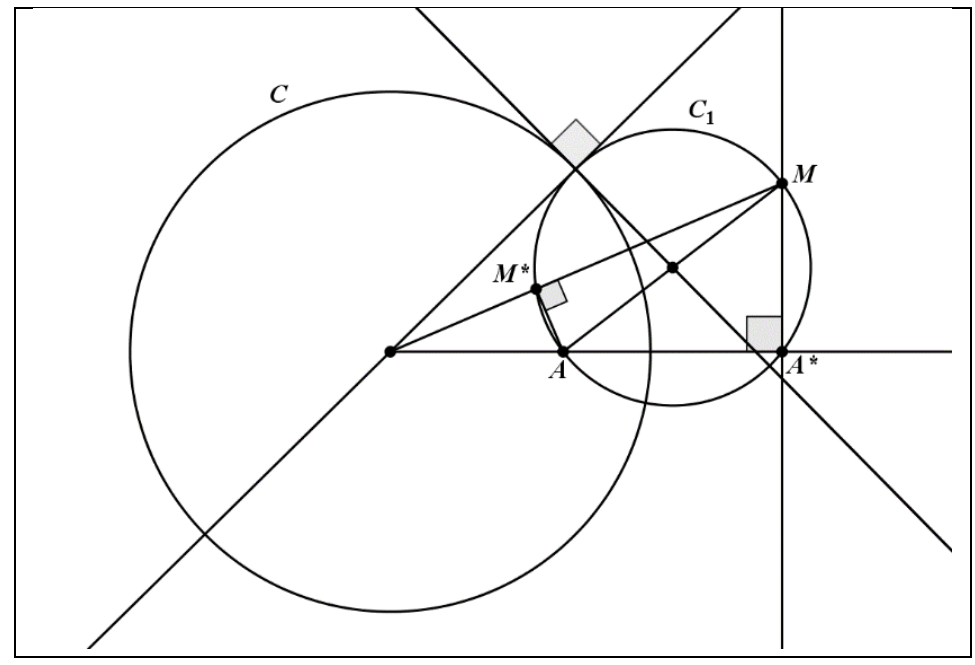

16. O ponto conjugado isogonal do baricentro é chamado de ponto Lemoine. As retas conectadas aos vértices com o ponto de Lemoine são chamadas de simedianas. Suponha que as retas tangentes nos pontos $B$ e $C$ em relação a circunferência circunscrita ao triângulo $A B C$ se intersectam no ponto $P$. Prove que $\overleftrightarrow{A P}$ é uma simediana do triângulo $A B C$. 


\section{Solução.}

Seja $Q$ o ponto de interseção das retas $\overleftrightarrow{A P}$ e $\overleftrightarrow{B C}$, e seja $Q_{1}$ um ponto da reta $\overleftrightarrow{B C}$ tal que a reta $\overleftrightarrow{A Q_{1}}$ seja isogonal à reta $\overleftrightarrow{A Q}$ no triângulo $A B C$. Com isso,

$$
\angle Q_{1} A C=\angle B A Q \text { e } \angle B A Q_{1}=\angle Q A C .
$$

Para um ponto arbitrário $X$ do segmento $B C$, a lei dos senos aplicada aos triângulos $B A X$ e $X A C$, nos dá

$$
\begin{aligned}
& \frac{\overline{B X}}{\bar{X} \bar{C}}=\frac{\overline{B X}}{\overline{A X}} \overline{\overline{X X}} \\
= & \frac{\operatorname{sen}(\angle B A X)}{\operatorname{sen}(\angle A B X)} \frac{\operatorname{sen}(\angle A C X)}{\operatorname{sen}(\angle X A C)} \\
= & \frac{\operatorname{sen}(\angle A C X)}{\operatorname{sen}(\angle A B X)} \frac{\operatorname{sen}(\angle B A X)}{\operatorname{sen}(\angle X A C)} \\
= & \overline{\overline{A B}} \frac{\operatorname{sen}(\angle B A X)}{\operatorname{sen}(\angle X A C)} .
\end{aligned}
$$

Fazendo $X=Q$ e $X=Q_{1}$, respectivamente, e substituindo em (2), obtemos:

$$
\frac{\overline{B Q}}{\overline{Q C}}=\frac{\overline{A B}}{\overline{A C}} \frac{\operatorname{sen}(\angle B A Q)}{\operatorname{sen}(\angle Q A C)}
$$

e

$$
\frac{\overline{B Q_{1}}}{\overline{Q_{1} C}}=\frac{\overline{A B}}{\overline{A C}} \frac{\operatorname{sen}\left(\angle B A Q_{1}\right)}{\operatorname{sen}\left(\angle Q_{1} A C\right)}
$$

Multiplicando (3) por (4) e usando (1), obtemos

$$
\frac{\overline{B Q}}{\overline{Q C}} \frac{\overline{B Q_{1}}}{\overline{Q_{1} C}}=\frac{\overline{A B}}{\overline{A C}} \frac{\operatorname{sen}(\angle B A Q)}{\operatorname{sen}(\angle Q A C)} \frac{\overline{A B}}{\overline{A C}} \frac{\operatorname{sen}\left(\angle B A Q_{1}\right)}{\operatorname{sen}\left(\angle Q_{1} A C\right)}=\frac{\overline{A B}^{2}}{\overline{A C}^{2}}
$$


Portanto, se provarmos que $\frac{\overline{B Q}}{\overline{Q C}}=\frac{\overline{A B}^{2}}{\overline{A C}^{2}}$, teremos imediatamente $\frac{\overline{B Q_{1}}}{\overline{Q_{1} C}}=1$, ou seja, que $Q_{1}$ é o ponto médio do segmento $B C$. Teremos então que a reta $\overleftrightarrow{A Q}$ é a conjugada isogonal da mediana, o que implica que a reta $\overleftrightarrow{A P}$ é uma simediana do triângulo $A B C$.

Como o ponto $P$ pertence às polares dos pontos $B$ e $C$, temos pelo, Teorema 3 , que os pontos $B$ e $C$ pertencem à polar do ponto $P$. Assim, a polar do ponto $P$ é a reta $\overleftrightarrow{B C}$. Seja o ponto $D$ a interseção da reta $\overleftrightarrow{B C}$ com a reta tangente à circunferência circunscrita ao triângulo $A B C$ no ponto $A$. O ponto $D$ pertence às polares dos pontos $A$ e $P$. Assim, a reta $\overleftrightarrow{A P}$ é a polar do ponto D.

Portanto, pelo Teorema 4, $H(B, C ; D, Q)$.

Vamos agora calcular a razão $\frac{\overline{B D}}{\overline{D C}}$. Uma vez que os triângulos $A B D$ e $C A D$ são semelhantes, temos

$$
\frac{\overline{B D}}{\overline{A D}}=\frac{\overline{A D}}{\overline{C D}}=\frac{\overline{A B}}{\overline{A C}},
$$

o que implica,

$$
\frac{\overline{B D}}{\overline{C D}}=\frac{\overline{B D}}{\overline{A D}} \frac{\overline{A D}}{\overline{C D}}=\frac{\overline{A B}^{2}}{\overline{A C}^{2}} .
$$

A relação

$$
\begin{aligned}
H(B, C ; D, Q) & \Rightarrow|R(B, C ; D, Q)|=1 \\
& \Rightarrow\left|\frac{\overline{B D}}{\overline{D C}} \frac{\overline{Q C}}{\overline{B Q}}\right|=1 \\
& \Rightarrow \frac{\overline{B Q}}{\overline{Q C}}=\left|\frac{\overline{A \bar{B}^{2}}}{\overline{A C^{2}}}\right|
\end{aligned}
$$




$$
\Rightarrow \frac{\overline{B Q}}{\overline{Q C}}=\frac{\overline{A B}^{2}}{\overline{A C}^{2}} .
$$

Aqui, foi usado (5) e o fato de as semirretas $\overrightarrow{B Q}$ e $\overrightarrow{Q C}$ terem o mesmo sentido.

\section{Problema 16}

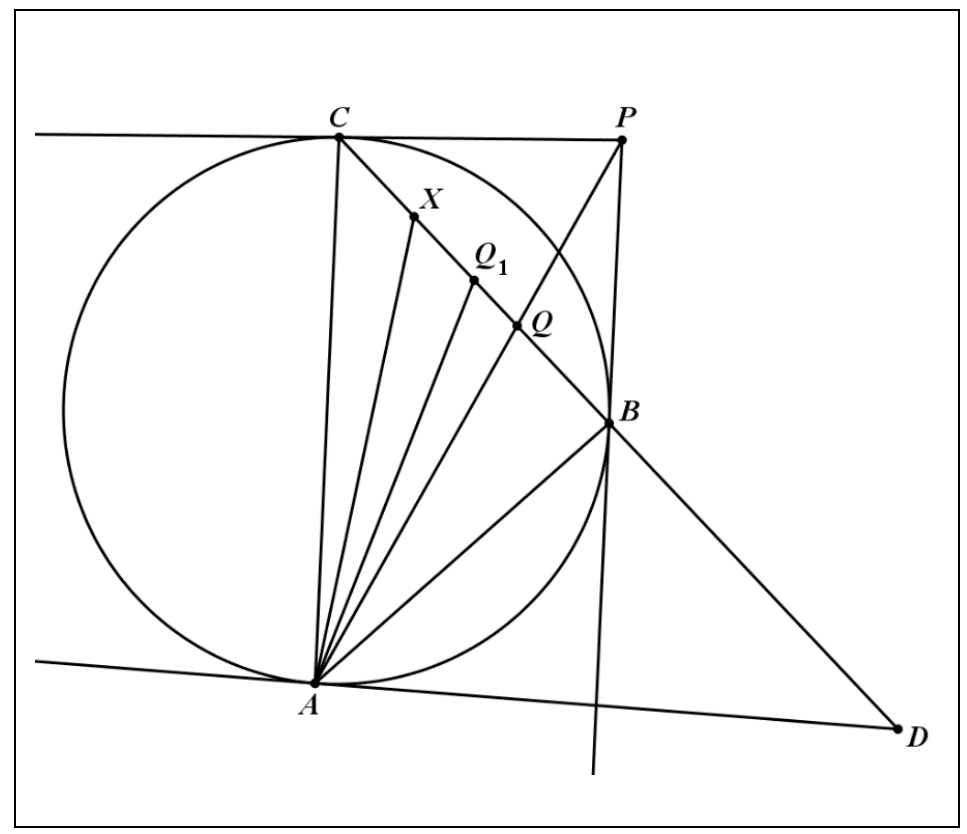

17. $O$ círculo inscrito de centro $I$ de um triângulo $A B C$ não isósceles é tangente aos lados $B C, C A$ e $A B$ em $A_{1}, B_{1}$ e $C_{1}$, respectivamente. As retas $\overleftrightarrow{A A_{1}}$ e $\overleftrightarrow{B B_{1}}$ se intersectam em $P$, as retas $\overleftrightarrow{A C}$ e $\overleftrightarrow{A_{1}} C_{1}$ em $M$, e as retas $\overleftrightarrow{B C}$ e $\overleftrightarrow{B_{1} C_{1}}$ em $N$. Prove que a reta $\overleftrightarrow{I P}$ é perpendicular à reta $\overleftrightarrow{M N}$.

\section{Solução.}

Pelo Teorema $3, B_{1}$ e $C_{1}$ pertencem à polar do ponto $A \mathrm{em}$ relação ao círculo inscrito ao triângulo $A B C$. Daí, a reta $\overleftrightarrow{B_{1} C_{1}}$ é 
a polar do ponto $A$ em relação ao círculo inscrito ao triângulo $A B C$.

O ponto $N$ pertence às retas $\overleftrightarrow{B C}$ e $\overleftrightarrow{B_{1} C_{1}}$, que são as polares dos pontos $A_{1}$ e $A$, respectivamente, em relação ao círculo inscrito ao triângulo $A B C$. Pelo Teorema 3 , os pontos $A_{1}$ e $A$ pertencem à polar do ponto $N$. Portanto, a reta $\overleftrightarrow{A A_{1}}$ é a polar de $N$. Analogamente, a reta $\overleftrightarrow{B B_{1}}$ é a polar do ponto $M$. Sendo $P=\overleftrightarrow{A A_{1}} \cap \overleftrightarrow{B B_{1}}$. Pelo Teorema 3 , os pontos $N$ e $M$ pertencem à polar de $P$. Assim, a reta $\overleftrightarrow{M N}$ é a polar de $P$.

Portanto, pela Definição 9 , a reta $\overleftrightarrow{M N}$ é perpendicular à reta $\overleftrightarrow{I P}$.

\section{Problema 17}

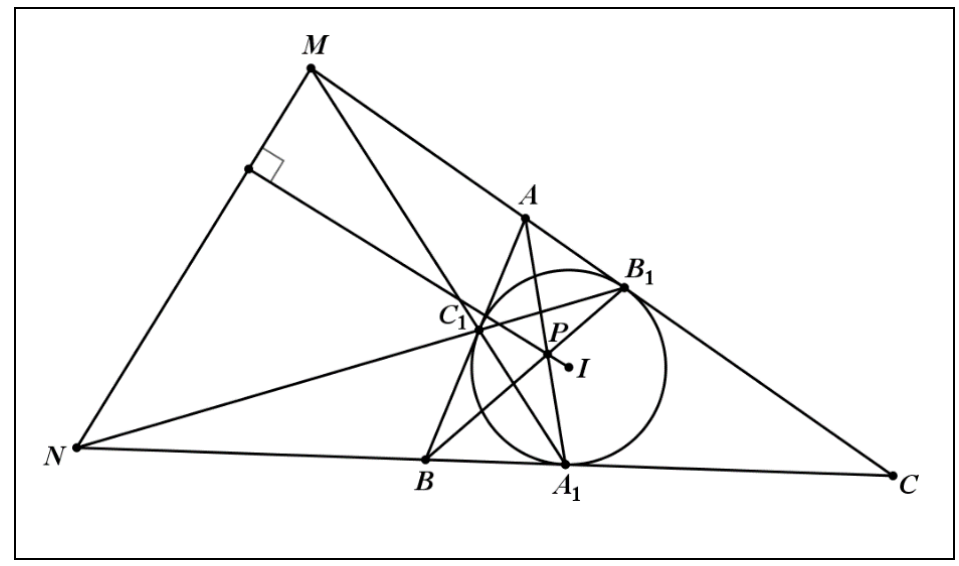

18. Seja $A B C D$ um quadrilátero circunscrito a uma circunferência. Sejam $M, N, P$ e $Q$ os pontos de tangência da circunferência com os lados $A B, B C, C D$ e $D A$, respectivamente. Prove que as retas $\overleftrightarrow{A C}, \overleftrightarrow{B D}, \overleftrightarrow{M P}$ e $\overleftrightarrow{N Q}$ se intersectam em um ponto. 


\section{Solução.}

Aplicando o Teorena de Brianchon no hexágono degenerado $A M B C P D$, concluímos que as retas $\overleftrightarrow{A C}, \overleftrightarrow{M P}$ e $\overleftrightarrow{B D}$ são concorrentes. Portanto, a reta $\overleftrightarrow{M P}$ contém o ponto de interseção das retas $\overleftrightarrow{A C}$ e $\overleftrightarrow{B D}$.

Analogamente, aplicando o Teorema de Brianchon no hexágono degenerado $A B N C D Q$, temos que a reta $\overleftrightarrow{N Q}$ contém o ponto de interseção das retas $\overleftrightarrow{A C}$ e $\overleftrightarrow{B D}$

Assim, as retas $\overleftrightarrow{A C}, \overleftrightarrow{B D}, \overleftrightarrow{M P}$ e $\overleftrightarrow{N Q}$ são concorrentes.

\section{Problema 18}

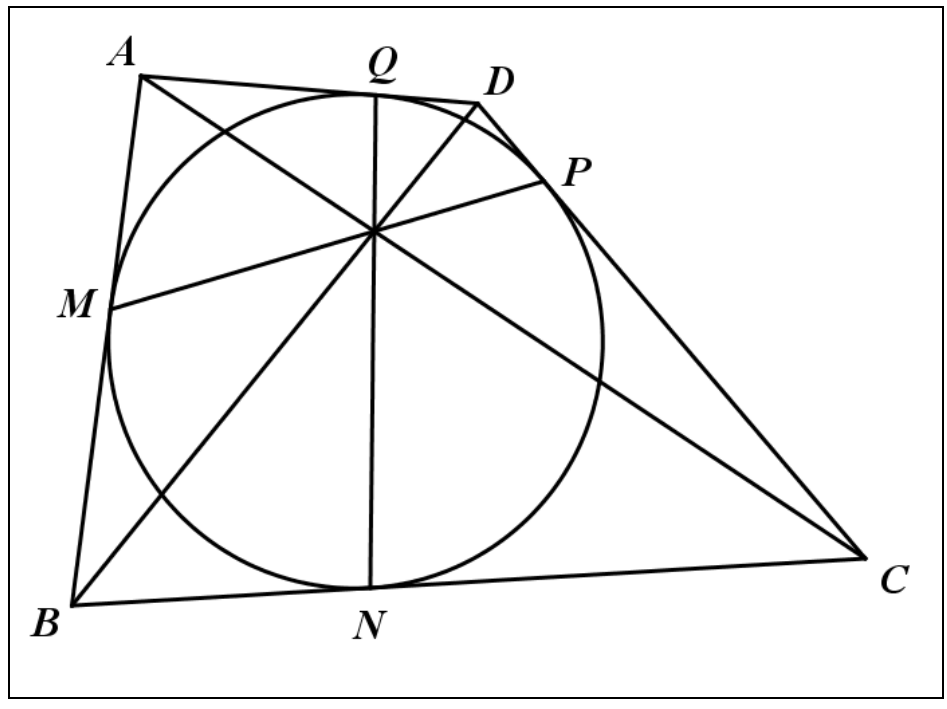

19. Seja $A B C D$ um quadrilátero incritível, cujas diagonais $A C$ e $B D$ se intersectam no ponto $O$, as extensões dos lados $A B$ e $C D$ em $E$, as tangentes à circunferência em $A$ e em $D$ se intersectam em $K$, e as tangentes à circuferência em $B$ e $C$ se intersectam em $L$. Prove que os pontos $E, K, O$, e $L$ são colineares. 


\section{Solução.}

O teorema de Brocard afirma que a polar do ponto $F=\overleftrightarrow{A D} \cap$ $\stackrel{\leftrightarrow}{B C}$ é a reta $f=\stackrel{\leftrightarrow}{E} \vec{O}$. Como a polar de um ponto pertencente à circunferência de inversão é igual à tangente nesse ponto, então $K=a \cap d$, onde $a$ e $d$ são as polares dos pontos $A$ e $D$, respectivamente. Pelo Teorema 3, temos $A \in k$ e $D \in k$, onde $k$ é a polar do ponto $K$ em relação à circunferência circunscrita ao quadrilátero $A B C D$. Assim, $k=\overleftrightarrow{A D}$. Como $F \in \overleftrightarrow{A D}=k$, o Teorema 3 implica que $K \in f$, onde $f$ é a polar do ponto $F$. Analogamente, podemos provar que $L \in f$. Portanto, os pontos $E, O, K$ e $L$ pertencem a $f$.

\section{Problema 19}

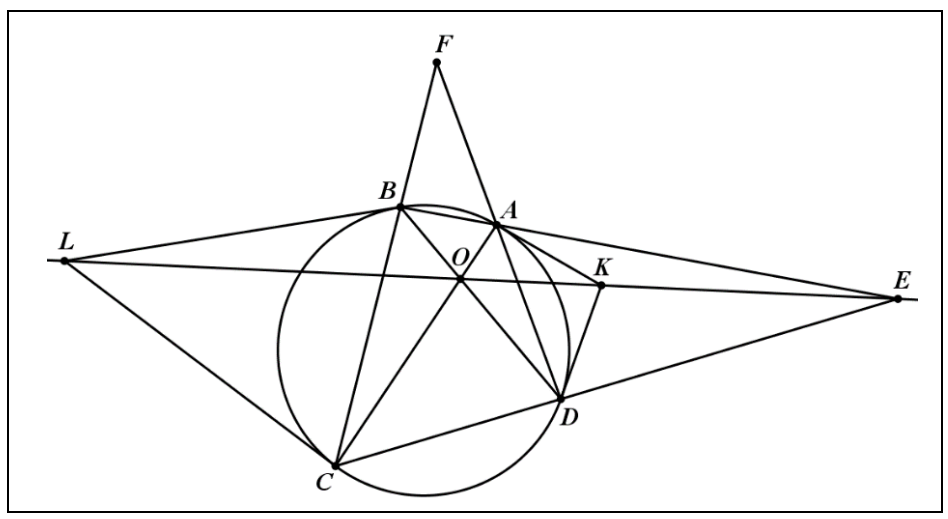

20. Seja $A B C D$ um quadrilátero cíclico. As retas $\overleftarrow{A} \vec{B}$ e $\stackrel{\leftarrow}{C}$ se intersectam no ponto $E$, e as diagonais $A C$ e $B D$ no ponto $F$. A circunferência circunscrita aos triângulos $A F D$ e $B F C$ se intersectam em $H$ e $F$. Prove que $\angle E H F=90^{\circ}$.

\section{Solução.}

Seja $G=\overleftrightarrow{A D} \cap \stackrel{\leftrightarrow}{B C}$ e seja $C$ a circunferência circunscrita ao quadrilátero $A B C D$. Denotamos por $C_{1}$ e $C_{2}$ as circunferências 
circunscritas aos triângulos $A D F$ e $B C F$, respectivamente. Obseve que a reta $\overleftrightarrow{A D}$ é o eixo radical das circunferência $C$ e $C_{1}$, a reta $\overleftrightarrow{B C}$ é o eixo radical de $C$ e $C_{2}$ e a reta $\overleftrightarrow{F H}$ é o eixo radical de $C_{1}$ e $C_{2}$.

É sabido que os três eixos radicais concorrem em um ponto, digamos $G$. Concluímos que os pontos $F, G$ e $H$ são colineares.

Sem perdar de generalidade, suponha que $F$ está entre $G$ e $H$. Usando os quadriláteros $A D F H$ e $B C F H$, inscritos nas circunferências $C_{1}$ e $C_{2}$, respectivamente, obtemos

$$
\angle D H F=\angle D A F=\angle D A C \text { e } \angle F H C=F B C=D B C .
$$

Portanto,

$\angle D H C=\angle D H F+\angle F H C=\angle D A C+\angle D B C=2 \angle D B C=$

$=\angle D O C$.

Assim, os pontos $D, C, H$ e $O$ pertencem a uma circunferência. Analogamente, os pontos $A, B, H$ e $O$ pertencem a uma outra circunferência. Denotamos por $C_{3}$ e $C_{4}$ as circunferências circunscritas aos quadriláteros $A B H O$ e $D C H O$, respctivamente. Temos que a reta $\overleftrightarrow{A B}$ é o eixo radical das circunferências $C \mathrm{e}$ $C_{3}$. Similarmente, as retas $\overleftrightarrow{C D}$ e $\overleftrightarrow{O H}$ são os eixos radicais dos pares de circunferências $C$ e $C_{4}, C_{3}$ e $C_{4}$, respectivamente. Assim, essas retas são concorrentes em um ponto $E=\overleftrightarrow{A B} \cap$ $\overleftrightarrow{C D} \cap \overleftrightarrow{O H}$. Com isso, os pontos $O, H$ e $E$ são colineares.

Usando o Teorema de Brocard temos $\overleftrightarrow{F H} \perp \overleftrightarrow{O E}$. Assim, como $\overleftrightarrow{F H}=\overleftrightarrow{G H}$ e $\overleftrightarrow{O E}=\overleftrightarrow{H E}$, obtemos que $\overleftrightarrow{G H} \perp \overleftrightarrow{H E}$. 


\section{Problema 20}

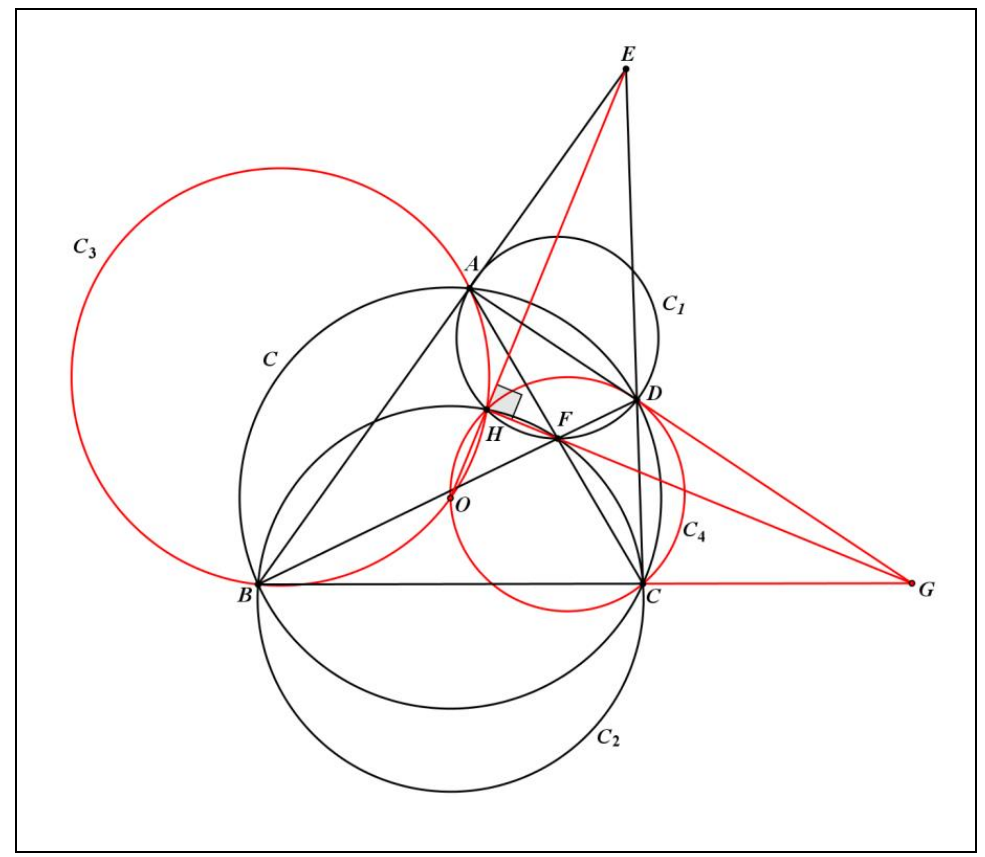




\section{CONSIDERAÇÕES FINAIS}

Conclui-se, através deste estudo, que vários problemas de Geometria Euclidiana podem ser resolvidos, de forma elegante, utilizando-se técnicas oriundas da Geometria Projetiva.

Espera-se que este livro seja utilizado por discentes, docentes e admiradores da Matemática na aprendizagem das técnicas aqui apresentadas, principalmente como instrumento preparatório para Olimpíadas de Matemática, contribuindo assim para o ensino da Geometria. 


\section{REFERÊNCIAS}

AUFFINGER, A.C.T.C.; VALENTIM, F.J.S. Introdução à Geometria Projetiva.Universidade Federal do Espírito Santo, 2013.

CASTRO, L.G.M. Introdução à Geometria Projetiva. Artigo baseado em aula ministrada na III Semana Olímpica. Piracicaba, 2000.

LUKIĆ, M. Projective Geometry. Disponível em: <http://w ww.imomath.com/index.php?options=330>. Acesso em: 12 abr. 2017.

POHOATA, C; VONK, J. The Monge-D'Alembert Circle Theorem. Disponível em: <https: //pt.scribd.com/document

REMOROV, A. Projective Geometry. Disponível em: < http://alexanderrem.weebly.com/ma th-competitions.html>. Acesso em: 20 abr. 2017. 


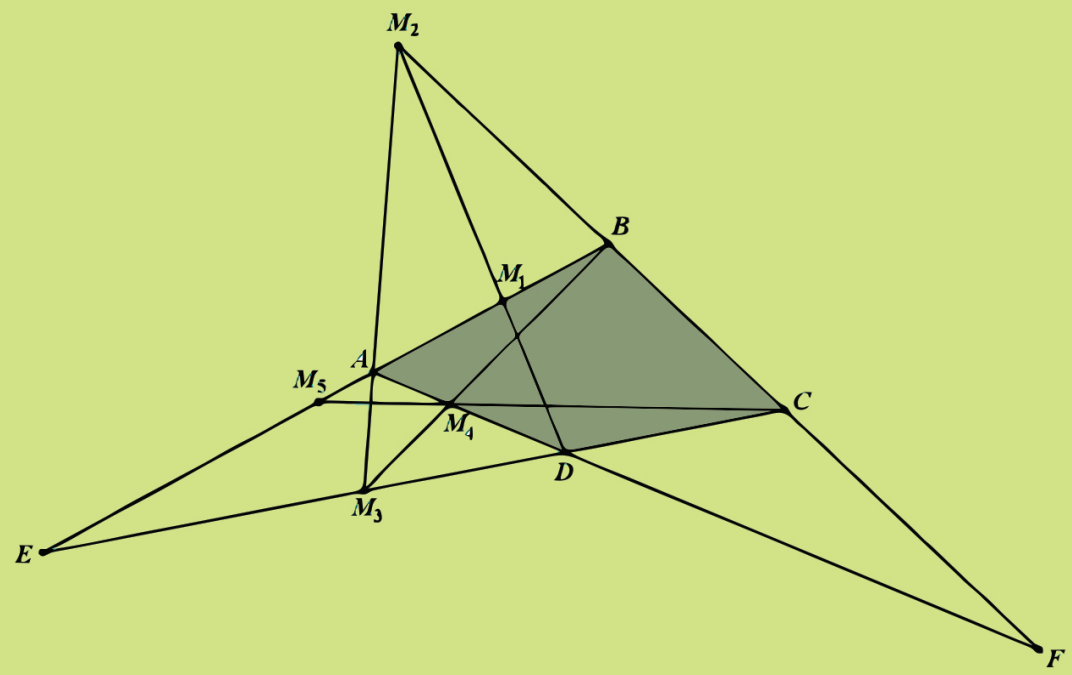

O presente livro visa mostrar a aplicabilidade das técnicas de Geometria Projetiva na resolução de problemas de Geometria Euclidiana. Inicialmente, traça-se um paralelo entre as duas geometrias e faz-se uma contextualização histórica da Geometria Projetiva. Em seguida são apresentadas versões euclidianas de definições, proposições e teoremas oriundos da Geometria Projetiva. Finalmente, são resolvidos problemas de Geometria Euclidiana usando-se técnicas de Geometria Projetiva. Tal enfoque possibilita aos discentes uma alternativa para a resolução de problemas geométricos, principalmente em olimpíadas matemáticas. 